COMMUNICATIONS IN

ANALYSIS AND GEOMETRY

Volume 12, Number 4, 733-791, 2004

\title{
Regularizing a Singular Special Lagrangian Variety
}

\section{Adrian Butscher}

Suppose $M_{1}$ and $M_{2}$ are two special Lagrangian submanifolds with boundary of $\mathbf{R}^{2 n}, n \geq 3$, that intersect transversally at one point $p$. The set $M_{1} \cup M_{2}$ is a singular special Lagrangian variety with an isolated singularity at the point of intersection. Suppose further that the tangent planes at the intersection satisfy an angle criterion (which always holds in dimension $n=3$ ). Then, $M_{1} \cup M_{2}$ is regularizable; in other words, there exists a family of smooth, minimal Lagrangian submanifolds $M_{\alpha}$ with boundary that converges to $M_{1} \cup M_{2}$ in a suitable topology. This result is obtained by first gluing a smooth neck into a neighbourhood of $M_{1} \cap M_{2}$ and then by perturbing this approximate solution until it becomes minimal and Lagrangian.

\section{Contents.}

1 Introduction . . . . . . . . . . . . . . . 734

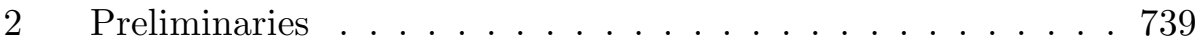

2.1 Outline of the Proof . . . . . . . . . . . 739

2.2 The Linearized Operator . . . . . . . . . . . . . 741

2.3 Boundary Conditions . . . . . . . . . . . . . . 743

3 The Approximate Solution . . . . . . . . . . . . . . . 743

3.1 The Local Regularization . . . . . . . . . . . . . 743

$3.2 \quad$ Construction of $\bar{M}_{\alpha} \quad \ldots \ldots \ldots \ldots$. . . . . . 747

$3.3 \quad$ Properties of $\bar{M}_{\alpha} \ldots \ldots \ldots \ldots$. . . . . . . 752

3.4 The First Neumann Eigenvalue of $\bar{M}_{\alpha} \ldots \ldots$. . . . 757

4 Deformations of $\bar{M}_{\alpha} \ldots \ldots \ldots \ldots \ldots$. . . . . . 758

4.1 Parametrizing Lagrangian Embeddings of $\bar{M}_{\alpha} \ldots \ldots 758$

4.2 The Weighted Schauder Norm . . . . . . . . . . . 762

5 Analysis of the Linearized Operator . . . . . . . . . . . . 770

5.1 Outline . . . . . . . . . . . . . 770

5.2 Second Eigenvalue Estimate for $\Delta_{\bar{M}_{\alpha}} \ldots \ldots$. . . . 771 
5.3 Injectivity Estimate for $\Delta_{\bar{M}_{\alpha}}$ in the Weighted Schauder Norms . . . . . . . . . . . . . 775

5.4 Injectivity Estimate for $P_{\alpha}$ in the Weighted Schauder Norms . . . . . . . . . . . . . . 777

5.5 Properties of the First Eigenfunction . . . . . . . 778

5.6 The Full Injectivity Estimate for $\mathrm{DF}_{\alpha}(0,0,0) \quad \ldots .782$

$5.7 \quad$ Surjectivity of the Linearized Operator . . . . . . . . 784

6 Solving the Nonlinear Problem . . . . . . . . . . . . 786

6.1 The Nonlinear Estimate . . . . . . . . . . 786

6.2 The Size of the $F_{\alpha}(0,0,0)$ Term $\ldots \ldots \ldots$. . . . 787

6.3 Invoking the Inverse Function Theorem . . . . . . . 788

\section{Introduction.}

A minimal Lagrangian submanifold of a symplectic manifold $\Sigma$ is at once minimal with respect to the metric of $\Sigma$ and Lagrangian with respect to the symplectic structure of $\Sigma$. Furthermore, when $\Sigma$ is a Calabi-Yau manifold, Harvey and Lawson showed in their seminal paper [6] that minimal Lagrangian submanifolds are also calibrated by the real part, up to phase, of the canonical, non-vanishing holomorphic $(n, 0)$-form $\zeta$ of $\Sigma$. (I. e. $\zeta$ is bounded above by the $n$-volume form and equality holds, up to a phase angle, only on the tangent spaces of minimal Lagrangian submanifolds.) A consequence of this property is that minimal Lagrangian submanifolds satisfy a relatively simple geometric PDE (simple relative to the equations of vanishing mean curvature, which they would satisfy by virtue of minimality alone). Namely, $M \subset \Sigma$ is minimal Lagrangian if and only if

$$
\begin{gathered}
\left.\operatorname{Im}\left(\mathrm{e}^{\mathrm{i} \theta} \zeta\right)\right|_{M}=0 \\
\left.\omega\right|_{M}=0,
\end{gathered}
$$

for some real number $\theta$. Here, $\omega$ is the symplectic form of $\Sigma$. The calibration form of $M$ is in this case $\operatorname{Re}\left(\mathrm{e}^{\mathrm{i} \theta} \zeta\right)$. The term special Lagrangian refers to those $M$ whose calibrating angle $\theta$ vanishes.

Many researchers have exploited the geometric structure implicit in the calibration condition in order to tackle questions related to the existence and properties of minimal Lagrangian submanifolds. Harvey and Lawson themselves produced several examples of minimal Lagrangian submanifolds and gave certain general constructions of such objects. More recently, Schoen 
and Wolfson [18] have been working on questions of existence of special Lagrangian submanifolds using variational techniques. Current developments in the mathematical foundations of string theory, in the form of mirror symmetry and the Strominger-Yau-Zaslow conjecture [17], have greatly stimulated further investigation into special Lagrangian submanifolds in the hopes of understanding the moduli spaces of these objects. McLean [16] began the study of deformations of smooth special Lagrangian submanifolds and Hitchin [8] deduced certain properties of the moduli space of such deformations. Compactification of this moduli space (an important ingredient in mirror symmetry), however, requires understanding the singularities that can arise amongst special Lagrangian submanifolds. Harvey and Lawson contributed a number of examples of singular special Lagrangian varieties, while Haskins [7] extended these results by constructing new examples of special Lagrangian cones and their desingularizations. Many further advances on the topic of singular special Lagrangian submanifolds are presented in papers by Joyce (for a summary, see [9]).

Despite these advances, still relatively little is known about singularities of special Lagrangian submanifolds and research in this domain continues. This paper investigates singular Lagrangian geometry in a setting related to the one in which mirror symmetry takes place. That is, this paper studies the nature of the singular special Lagrangian submanifold with boundary of $\mathbf{R}^{2 n}, n \geq 3$, formed by the union of two otherwise smooth special Lagrangian submanifolds $M_{1}$ and $M_{2}$ that intersect transversally in one point $p$, and whose tangent cone at $p$ satisfies an angle criterion to be explained shortly. The union $M$ of the two smooth submanifolds is singular at the intersection point (which is isolated for reasons of transversality) and the tangent cone there is just the union of the two tangent planes of the constituent smooth submanifolds. The question this paper partially answers in the affirmative in the Main Theorem, to be stated precisely below, is whether $M$ can be realized as the limit of a sequence of smooth minimal Lagrangian submanifolds boundary, thus placing $M$ in the compactification of the moduli space of minimal Lagrangian submanifolds with boundary of $\mathbf{R}^{2 n}$. It is hoped that this result as well as the methods used in its proof will shed some light on the analogous question of intersections of special Lagrangian submanifolds in compact Calabi-Yau manifolds.

Before stating precisely the result that will be proved in this paper, it is necessary to mention that the boundary behaviour of the regularizing family will play a critical role in the forthcoming analysis. The way in which the boundary of $M$ will be controlled is to use a submanifold of $\mathbf{R}^{2 n}$ that will 
be called a scaffold.

Definition 1. Let $M$ be a submanifold of $\mathbf{R}^{2 n}$ with boundary $\partial M$ and inward unit normal vector field $Z \in \Gamma\left(T_{\partial M} M\right)$. A scaffold for $M$ is a smooth submanifold $W$ of $\mathbf{R}^{2 n}$ with the following properties:

1. $\partial M \subset W$

2. $Z \in \Gamma\left(T_{\partial M} W\right)^{\omega}\left(V^{\omega}\right.$ is the symplectic orthogonal complement of $\left.V\right)$;

3. The normal bundle of $W$ is trivial.

The idea of the scaffold is first introduced in [2] and [3] in the context of a different problem concerning deformations of smooth minimal Lagrangian submanifolds with boundary and is discussed in detail there. Condition (2) might seem slightly unnatural, but it considerably simplifies the proof of the Main Theorem. Nevertheless, one expects the Main Theorem to hold under a more general transversality condition.

The precise statement of the theorem to be proved in this paper that incorporates the boundary behaviour of the regularization is the following.

Main Theorem. Suppose $M_{1}$ and $M_{2}$ are two special Lagrangian submanifolds with boundary of $\mathbf{R}^{2 n}, n \geq 3$, that intersect transversally at one point $p$. Furthermore, suppose that the tangent planes of $M_{1}$ and $M_{2}$ satisfy the angle criterion (described below) at the point $p$ and let $W$ be a scaffold for $M$ that is also a codimension 2, symplectic submanifold of $\mathbf{R}^{2 n}$. Define $M=M_{1} \cup M_{2}$. Then there exists a family $M_{\alpha}$ of smooth, minimal Lagrangian submanifolds with boundary and a family of symplectic, codimension-two submanifolds $W_{\alpha}$ such that the following results hold.

1. $M_{\alpha} \rightarrow M$ in some suitable topology;

2. $W_{\alpha} \rightarrow W$ in the same topology;

3. $\partial M_{\alpha} \subseteq W_{\alpha}$ for every $\alpha$.

REMARKS: (1) If $M$ is any Lagrangian submanifold of $\mathbf{R}^{2 n}$ with boundary then there always exists a symplectic, codimension-two scaffold for $M$ : for instance, it can be constructed by exponentiating a sufficiently small neighbourhood $\mathcal{U}$ of the zero section of the bundle $T(\partial M) \oplus J T(\partial M)$. (2) Due to technical reasons that make their appearance only in Section 6 , the argument used to prove the Main Theorem fails in dimension $n=2$. It is 
unfortunately not yet clear to the Author how to proceed in the $n=2$ case using the techniques developed herein; however, the $n=2$ case is true in certain circumstances for other reasons. See the end of this section for an explanation.

The angle criterion that $M_{1}$ and $M_{2}$ must satisfy can be explained as follows. It can be shown that if $P_{1}$ and $P_{2}$ are any two transversally intersecting Lagrangian planes in $\mathbf{R}^{2 n}$, then, up to a global unitary transformation of $\mathbf{R}^{2 n}$, there exists an orthonormal Darboux basis $E_{1}, \ldots, E_{n}$ and $J E_{1}, \ldots, J E_{n}$ of $\mathbf{R}^{2 n}$ and a unique set of angles $\theta_{i}$, where

$$
\theta_{1} \in\left[\frac{\pi}{2}, \pi\right) \quad \text { and } \quad \theta_{i} \in\left[0, \frac{\pi}{2}\right) \text { for } i=2, \ldots, n,
$$

such that

$$
P_{1}=\operatorname{span}\left\{E_{1}, \ldots, E_{n}\right\}
$$

and

$$
P_{2}=\operatorname{span}\left\{\cos \theta_{1} E_{1}+\sin \theta_{1} J E_{1}, \ldots, \cos \theta_{n} E_{n}+\sin \theta_{n} J E_{n}\right\} .
$$

The existence of this basis follows from standard symplectic linear algebra and can be found in $[3,14]$ as well as [5], for example. Since an orthonormal Darboux basis is by definition holomorphic, the form $\mathrm{d} z$ becomes $\left(E_{1}^{*}+\right.$ $\left.\mathrm{i} J E_{1}^{*}\right) \wedge \cdots \wedge\left(E_{n}^{*}+\mathrm{i} J E_{n}^{*}\right)$ in these coordinates, where $E_{i}^{*}$ denotes the dual 1-form corresponding to the vector $E_{i}$. Thus by applying the calibrating form $\operatorname{Im} \mathrm{d} z$ to the pair of planes $P_{1}$ and $P_{2}$, one sees that they are special Lagrangian if and only if $\theta_{1}+\cdots+\theta_{n}=m \pi$ for some positive integer $m$. The angle criterion for $P_{1}$ and $P_{2}$ is that the characteristic angles satisfy $\theta_{1}+\cdots+\theta_{n}=\pi$. Note that the angle criterion need not hold for a general pair of intersecting special Lagrangian planes, though it is always satisfied in dimensions $n=2$ and 3 for numerical reasons (i. e. as a result of (2)). The angle criterion that the pair of submanifolds $M_{1} \cup M_{2}$ must satisfy in order for the Main Theorem to hold is that the characteristic angles of the pair of special Lagrangian planes that form the tangent cone $T_{p} M_{1} \cup T_{p} M_{2}$ at the singularity $p$ satisfy the angle criterion explained above.

The Author would like to thank Yng-Ing Lee for pointing out the necessity and importance of the angle criterion in the proof of the Main Theorem. The angle criterion is a necessary requirement on $M_{1}$ and $M_{2}$ because only when it holds, is it known that there exists a local regularization for the tangent cone $T_{p} M_{1} \cup T_{p} M_{2}$ - that is, a smooth submanifold $N$ with two 
ends, asymptotic to $T_{p} M_{1}$ and $T_{p} M_{2}$ respectively. This object will be called a Lawlor neck, and was discovered by Lawlor in [13], but see also [5] for a more thorough treatment. The first step of the proof of the Main Theorem is to glue a rescaled piece of this Lawlor neck into the neighbourhood of $p$ in order to obtain a smooth, approximately minimal Lagrangian submanifold. Thus without the angle criterion, this first step can not be undertaken and the proof of the Main Theorem does not get off the ground. When the angle criterion fails for $M_{1}$ and $M_{2}$, one will first have to find a new local regularization of the tangent cone at the singularity (one which is necessarily not of Lawlor's type, as indicated by Lee) before being able to apply the techniques of this paper to obtain a version of the Main Theorem valid in this case. The reader should consult Lee's paper [14] for a more complete discussion of this issue, and how it relates to her work on immersed, selfintersecting special Lagrangian submanifolds without boundary in general Calabi-Yau manifolds.

Remark on the $n=2$ CASE: Suppose $M_{1}$ and $M_{2}$ are two special Lagrangian submanifolds with boundary in $\mathbf{R}^{4}$ that intersect transversally at one point. As will become clear in Section 6.3, the techniques used to prove the Main Theorem fail in this situation. However, in certain cases, another method provides a solution. Let $x^{1}, x^{2}, y^{1}, y^{2}$ denote the standard coordinates of $\mathbf{R}^{4}$. It is easy to verify that the coordinate transformation

$$
\left(x^{1}, x^{2}, y^{1}, y^{2}\right) \mapsto\left(x^{1}, y^{1},-x^{2}, y^{2}\right)
$$

applied to $M_{1}$ and $M_{2}$ produces submanifolds $M_{1}^{\prime}$ and $M_{2}^{\prime}$ intersecting transversally at one point whose tangent spaces are invariant under the complex structure of $\mathbf{R}^{4}$. In other words, $M_{1}^{\prime}$ and $M_{2}^{\prime}$ are intersecting Riemann surfaces. Thus the question of regularizing $M_{1} \cup M_{2}$ becomes a problem in two complex variables. If $M_{1}^{\prime}$ and $M_{2}^{\prime}$ can be represented as the zero sets of holomorphic functions $h_{1}$ and $h_{2}$, then the variety $M^{\prime}=M_{1}^{\prime} \cup M_{2}^{\prime}$ can be regularized using complex analytic techniques. Indeed, $M^{\prime}$ can be represented as the zero set of the holomorphic function $h=h_{1} \cdot h_{2}$, and due to the nature of the singularity (without loss of generality, occuring at the origin) of $M^{\prime}$, there are coordinates $(z, w)$ for a neighbourhood of the origin in which $h(z, w)=z \cdot w \cdot \tilde{h}$, where $\tilde{h}(0,0) \neq 0$. Consequently, the function $h_{\varepsilon}=h+\varepsilon$ is holomorphic and has smooth zero section, provided $\varepsilon$ is sufficiently small. The Riemann surfaces $M_{\varepsilon}^{\prime}=h_{\varepsilon}^{-1}(0)$ thus regularize $M^{\prime}$. Changing coordinates back to the original ones then provides the desired regularization of $M$. This method is not always available since $M_{1}^{\prime}$ and $M_{2}^{\prime}$ may not be representable globally as the zero sets of holomorphic functions. In this case, 
new methods will have to be developed to solve the regularization problem, and these are, at the moment, beyond the scope of this paper.

\section{Preliminaries.}

\subsection{Outline of the Proof.}

The regularization $M_{\alpha}$ of the singular variety $M=M_{1} \cup M_{2}$ will be constructed by applying gluing techniques $[10,11,12,15]$ in combination with the Inverse Function Theorem to a system of differential equations associated to the geometric equations (1) whose solutions correspond to minimal Lagrangian submanifolds near $M$. The purpose of this section is to describe this procedure in general terms.

The preliminary step in the proof of the Main Theorem is to replace $M$ by a family of smooth, embedded, Lagrangian submanifolds $\bar{M}_{\alpha}$ approximating $M$, i. e. by submanifolds $\bar{M}_{\alpha}$ that are almost special Lagrangian in a precise sense and that converge to $M$ in a suitable topology. These approximating submanifolds will be constructed in detail in Section 3. The reason for replacing $M$ by $\bar{M}_{\alpha}$ is that the point of non-smoothness of $M$ makes it difficult to apply PDE techniques directly to $M$. The question of finding a minimal Lagrangian submanifold near $M$ can be converted to solving a PDE on $\bar{M}_{\alpha}$ as follows.

Denote by $\bar{h}_{\alpha}: \bar{M}_{\alpha} \rightarrow \mathbf{R}^{2 n}$ the canonical embedding of $\bar{M}_{\alpha}$ and let $\mathcal{B}$ be a Banach space that parametrizes a subset of $C^{1, \beta}$ Lagrangian embeddings of $\bar{M}_{\alpha}$ into $\mathbf{R}^{2 n}$ that are near $\bar{h}_{\alpha}$ in some suitable topology. In other words, suppose that there is a continuous map $\Phi: \mathcal{B} \rightarrow C^{1, \beta}\left(\operatorname{Emb}\left(\bar{M}_{\alpha}, \mathbf{R}^{2 n}\right)\right)$ into the immersions of $\bar{M}_{\alpha}$ into $\mathbf{R}^{2 n}$ with $\Phi(0)=\bar{h}_{\alpha}$ and such that $\Phi(x)\left(\bar{M}_{\alpha}\right)$ is Lagrangian for every $x \in \mathcal{B}$. The parametrization and the Banach space will be specified in Section 4 and will be such that if $\|x\|_{\mathcal{B}}$ is sufficiently small, then $\Phi(x)\left(\bar{M}_{\alpha}\right)$ is smooth as well as embedded. The first of the two minimal Lagrangian equations (1), namely that $\Phi(x)^{*} \omega=0$, is automatically satisfied because each $\Phi(x)$ is a Lagrangian embedding, by definition. Hence it is sufficient to analyze only the second of the two equations. Recall that the canonical, non-vanishing holomorphic $(n, 0)$-form of $\mathbf{R}^{2 n}$ is $\zeta=\mathrm{d} z^{1} \wedge$ $\cdots \wedge \mathrm{d} z^{n}$ in standard holomorphic coordinates. This form will henceforth be abbreviated by $\mathrm{d} z$. Consider now the differential operator between Banach spaces defined by the second equation of (1): namely, the map

$$
F_{\alpha}: \mathcal{B} \times \mathbf{R} \longrightarrow C^{0, \beta}\left(\bar{M}_{\alpha}\right)
$$


given by

$$
F_{\alpha}(x, \theta)=\left\langle\Phi(x)^{*}\left(\operatorname{Im}\left(\mathrm{e}^{\mathrm{i} \theta} \mathrm{d} z\right)\right), \operatorname{Vol}_{\bar{M}_{\alpha}}\right\rangle_{\bar{M}_{\alpha}}
$$

for any $x \in \mathcal{B}$. Here, $\operatorname{Vol}_{\bar{M}_{\alpha}}$ is the induced volume form of $\bar{M}_{\alpha}$ and $\langle\cdot, \cdot\rangle_{\bar{M}_{\alpha}}$ is the induced inner product on the $n$-forms of $\bar{M}_{\alpha}$.

A solution of the equation $F_{\alpha}(x, \theta)=0$ for some $(x, \theta)$ near the origin in $\mathcal{B} \times \mathbf{R}$ corresponds to an embedded minimal Lagrangian submanifold $M_{\alpha}=\Phi(x)\left(\bar{M}_{\alpha}\right) \subset \mathbf{R}^{2 n}$ with calibration angle $\theta$ that is near $\bar{M}_{\alpha}$, and thus near $M$ as well. Solutions are found by invoking the following version of the Inverse Function Theorem (see [1] for the proof).

Theorem 2 (Inverse Function Theorem). Let $F: \mathcal{B} \longrightarrow \mathcal{B}^{\prime}$ be a $C^{1}$ map between Banach spaces and suppose that the linearization $\mathrm{DF}(0)$ of $F$ at 0 is an isomorphism. Moreover, suppose $F$ satisfies the estimates:

1. $\|\mathrm{D} F(0) x\|_{\mathcal{B}^{\prime}} \geq C_{L}\|x\|_{\mathcal{B}}$ for any $x \in \mathcal{B}$,

2. $\|\mathrm{D} F(0) y-\mathrm{D} F(x) y\|_{\mathcal{B}^{\prime}} \leq C_{N}\|x\|_{\mathcal{B}}\|y\|_{\mathcal{B}}$ for all $x$ sufficiently near 0 and for any $y \in \mathcal{B}$,

where $C_{L}$ and $C_{N}$ are constants independent of $x$ and $y$. Let $r \leq C_{L} / 2 C_{N}$. Then there exist neighbourhoods $\mathcal{U}$ of 0 and $\mathcal{V}$ of $F(0)$ so that $F: \mathcal{U} \longrightarrow \mathcal{V}$ is a $C^{1}$ diffeomorphism and $\mathcal{V}$ contains the ball $B_{r C_{L} / 2}(F(0))$. Furthermore, $B_{r C_{L} / 2}(F(0))$ is in the image of the ball $B_{r}(0)$ under $F$.

The submanifold $\bar{M}_{\alpha}$ only approximates $M$ and is not necessarily minimal Lagrangian; thus $F_{\alpha}(0,0)$ is not equal to 0 . But according to the theorem above, the equation $F_{\alpha}(x, \theta)=0$ can be solved for $(x, \theta)$ near $(0,0)$ if 0 belongs to the ball $B_{r C_{L} / 2}(F(0))$; that is, if

$$
\left\|F_{\alpha}(0,0)\right\|_{C^{0, \beta}\left(\bar{M}_{\alpha}\right)} \leq r C_{L} / 2,
$$

and the theorem asserts that the solution satisfies $\|(x, \theta)\|_{\mathcal{B} \times \mathbf{R}} \leq r$.

The Main Theorem will be proved by verifying that the inequality (4) holds for the choices of approximating submanifold $\bar{M}_{\alpha}$ and parametrization of nearby Lagrangian embeddings made in Sections 2 and 3. In Section 4, the constant $C_{L}(\alpha)$ (depending of course on $\alpha$ ) will be estimated by analyzing the linearization at $(0,0)$ of the operator $F_{\alpha}$ and in Section 5 , the proof will be completed by deriving the constant $C_{N}(\alpha)$ (also depending on $\alpha$ ), invoking the Inverse Function Theorem, and using the estimate $\|(x, \theta)\|_{\mathcal{B} \times \mathbf{R}} \leq r$ to be used to guarantee the smoothness of the solution. 


\subsection{The Linearized Operator.}

The linearized operator $\mathrm{D} F_{\alpha}(0,0)$ will clearly play a central role in the forthcoming analysis. It will be helpful, at this point, to derive a general expression for this operator in terms of the parametrization $\Phi$, since its specific form will guide the course of the proof. The following calculations are based on McLean's work on perturbations of smooth, special Lagrangian submanifolds [16].

Begin with two preliminary observations. First, recall that $\Phi(t x)$ is a family of Lagrangian embeddings. Hence, $\Phi(t x)^{*} \omega=0$ for every $t$. Differentiating this expression in $t$ and using the Lie derivative formula $\left.\left.\mathcal{L}_{V} \eta=\mathrm{d}(V\rfloor \eta\right)+V\right\rfloor \mathrm{d} \eta$ as in McLean's paper produces the expression

$$
\left.\mathrm{d} \bar{h}_{\alpha}^{*}\left(V_{x}\right\rfloor \omega\right)=0,
$$

where $V$ is the deformation vector field associated to $\Phi(t x)$ given by

$$
V_{x}=\left.\frac{\mathrm{d}}{\mathrm{d} t} \Phi(t x)\right|_{t=0} .
$$

Second, if the parametrization $\Phi$ is chosen such that the closedness of the form $\left.\bar{h}_{\alpha}^{*}\left(V_{x}\right\rfloor \omega\right)$ implies exactness, then there is a function $H_{x}: \bar{M}_{\alpha} \rightarrow \mathbf{R}$ so that $\left.\bar{h}_{\alpha}^{*}\left(V_{x}\right\rfloor \omega\right)=\mathrm{d} H_{x}$. Refer to $H_{x}$ as the Hamiltonian function associated to $x$.

Next, it is necessary to remind the reader of the Lagrangian angle function that is defined on any Lagrangian submanifold of a C-Y manifold $\Sigma$ with canonical, non-vanishing holomorphic $(n, 0)$-form $\zeta$. First, let $e_{1}, \ldots, e_{n}$ be an orthonormal basis for any Lagrangian subspace of $T_{x} \Sigma$. It can be shown that the complex number $\zeta\left(e_{1}, \ldots, e_{n}\right)$ is independent of the particular basis and always has modulus equal to one. See [6, page 89] for details. Consequently, if $M$ is a Lagrangian submanifold of $\Sigma$ and $e_{1}, \ldots, e_{n}$ is an orthonormal basis for $T_{x} M$, then there is an angle $\theta(x)$ that satisfies

$$
\mathrm{e}^{-\mathrm{i} \theta(x)} \equiv \zeta\left(e_{1}, \ldots, e_{n}\right) .
$$

If $M$ is minimal Lagrangian and has calibration angle $\theta$ then $\theta(x)=\theta$ for all $x \in M$. The significance of the possibly multi-valued function $\theta$ lies in the fact that its differential $\mathrm{d} \theta$ is well-defined and satisfies the relation

$$
\left.\mathrm{d} \theta=-\vec{H}_{M}\right\rfloor \omega,
$$

where $\vec{H}_{M}$ is the mean curvature vector of $M$.

The linearization of the operator $F_{\alpha}$ can now be phrased using this terminology. 
Proposition 3. Let $H_{x}$ and $V_{x}$ be the Hamiltonian function and the deformation vector field associated to $x$ via the parametrization $\Phi$ as discussed above. The linearization of the operator $F_{\alpha}: \mathcal{B} \times \mathbf{R} \rightarrow C^{0, \beta}\left(\bar{M}_{\alpha}\right)$ at $(0,0)$ in the direction $(x, a) \in \mathcal{B} \times \mathbf{R}$ is given by

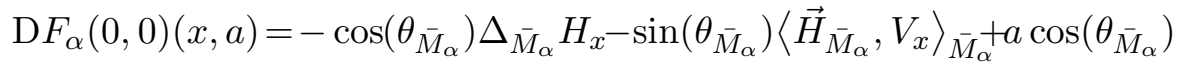

where $\vec{H}_{\bar{M}_{\alpha}}$ and $\theta_{\bar{M}_{\alpha}}$ are the mean curvature and the Lagrangian angle of $\bar{M}_{\alpha}$.

Proof. The linearization of $F_{\alpha}$ at $(0,0)$ in the direction of $x \in \mathcal{B}$ is defined as the quantity

$$
\left.\mathrm{D} F_{\alpha}(0,0) x \equiv \frac{\mathrm{d}}{\mathrm{d} t} F_{\alpha}(t x, 0)\right|_{t=0} .
$$

Thus the calculations performed in Mclean's paper [16], modified for the case when the Lagrangian angle function is not identically zero, imply that

$$
\begin{aligned}
\mathrm{D} F_{\alpha}(0,0)(x, 0) & =\left\langle\left.\frac{\mathrm{d}}{\mathrm{d} t} \Phi(t x)^{*} \mathbf{I m} \mathrm{d} z\right|_{t=0}, \operatorname{Vol}_{\bar{M}_{\alpha}}\right\rangle_{\bar{M}_{\alpha}} \\
& \left.=\left\langle\mathrm{d} \star\left(\cos \left(\theta_{\bar{M}_{\alpha}}\right) \bar{h}_{\alpha}^{*}\left(V_{x}\right\rfloor \omega\right)\right), \operatorname{Vol}_{\bar{M}_{\alpha}}\right\rangle_{\bar{M}_{\alpha}} \\
& =\left\langle\mathrm{d}\left(\cos \left(\theta_{\bar{M}_{\alpha}}\right) \star \mathrm{d} H_{x}\right), \operatorname{Vol}_{\bar{M}_{\alpha}}\right\rangle_{\bar{M}_{\alpha}} \\
& =-\cos \left(\theta_{\bar{M}_{\alpha}}\right) \Delta_{\bar{M}_{\alpha}} H_{x}+\sin \left(\theta_{\bar{M}_{\alpha}}\right)\left\langle\mathrm{d} \theta_{\bar{M}_{\alpha}} \wedge \star \mathrm{d} H_{x}, \operatorname{Vol}_{\bar{M}_{\alpha}}\right\rangle_{\bar{M}_{\alpha}} \\
& =-\cos \left(\theta_{\bar{M}_{\alpha}}\right) \Delta_{\bar{M}_{\alpha}} H_{x}+\sin \left(\theta_{\bar{M}_{\alpha}}\right)\left\langle\vec{H}_{\bar{M}_{\alpha}}, V_{x}\right\rangle_{\bar{M}_{\alpha}}
\end{aligned}
$$

using the relationship (7). Here, $\star$ and $\Delta_{\bar{M}_{\alpha}}$ are the Hodge star operator and the Laplacian of $\bar{M}_{\alpha}$ in the induced metric. The linearization of $F_{\alpha}$ in the $\theta$-direction can be calculated similarly. In fact, if $a \in \mathbf{R}$, then

$$
\begin{aligned}
\mathrm{D} F_{\alpha}(0,0)(0, a) & =\left\langle\left.\frac{\mathrm{d}}{\mathrm{d} t} \Phi(0)^{*} \operatorname{Im}\left(\mathrm{e}^{\mathrm{i} t a} \mathrm{~d} z\right)\right|_{t=0}, \operatorname{Vol}_{\bar{M}_{\alpha}}\right\rangle_{\bar{M}_{\alpha}} \\
& =a\left\langle\bar{h}_{\alpha}^{*} \mathbf{I m}(\mathrm{i} \mathrm{d} z), \operatorname{Vol}_{\bar{M}_{\alpha}}\right\rangle_{\bar{M}_{\alpha}} \\
& =a\left\langle\bar{h}_{\alpha}^{*} \operatorname{Re}(\mathrm{d} z), \operatorname{Vol}_{\bar{M}_{\alpha}}\right\rangle_{\bar{M}_{\alpha}} \\
& =a \cos \left(\theta_{\bar{M}_{\alpha}}\right)
\end{aligned}
$$

by definition of the angle function. Combining the two results above yields the desired expression for the linearization. 


\subsection{Boundary Conditions.}

Since the linearization of $F_{\alpha}$ at $(0,0)$ is equal to the Laplacian plus a lower order term, it is elliptic only when the functions in $\mathcal{B}$ satisfy appropriate boundary conditions. The purpose of this section is to show how the scaffold introduced in the statement of the Main Theorem brings this about.

Suppose that the boundary of $M$ lies on a scaffold $W$. Since the submanifold $\bar{M}_{\alpha}$ will differ from $M$ only in a small neighbourhood disjoint from the boundary, then $\partial \bar{M}_{\alpha}=\partial M$ and thus $W$ is a scaffold for $\bar{M}_{\alpha}$ as well. Let $\Phi: \mathcal{B} \rightarrow \mathbf{C}^{1, \beta}\left(\operatorname{Emb}\left(\bar{M}_{\alpha}, \mathbf{R}^{2 n}\right)\right)$ parametrize a subset of Lagrangian embeddings near $\bar{h}_{\alpha}$ as above. This time, however, suppose that each embedding confines the boundary of $\bar{M}_{\alpha}$ to $W$; in other words, suppose that $\Phi(x)\left(\partial \bar{M}_{\alpha}\right) \subset W$ for every $x \in \mathcal{B}$.

Consider now a one-parameter family $\Phi(t x)$ of such embeddings. Since $\Phi(t x)\left(\partial \bar{M}_{\alpha}\right) \subset W$ for every $t$, the deformation vector field $V_{x}$ from (5) must be tangent to $W$, which leads to the following result.

Proposition 4. Let $M$ be a Lagrangian submanifold of $\mathbf{R}^{2 n}$ and let $W$ be a scaffold for $M$. If $\phi^{t}: M \rightarrow \mathbf{R}^{2 n}$ is any Hamiltonian deformation of $M$ with Hamiltonian $H$ that satisfies $\phi_{H}^{t}(\partial M) \subset W$ for all $t$, then $H$ satisfies $\left.Z(H)\right|_{\partial M}=0$ where $Z$ is the inward unit normal vector field of $\partial M$.

Proof. The deformation vector field $V$ of $\phi$ must be parallel to $W$ along $\partial M$. But according to the definition of a scaffold, $Z \in\left(T_{x} W\right)^{\omega}$ for every $x \in \partial L$. Therefore $\left.\omega(Z, V)\right|_{\partial M}=0$. Since $V$ is a Hamiltonian vector field, this equality is equivalent to $\left.Z(H)\right|_{\partial M}=0$.

Thus if functions in $\mathcal{B}$ confine $\partial \bar{M}_{\alpha}$ to the scaffold $W$, then they satisfy Neumann boundary conditions.

\section{The Approximate Solution.}

\subsection{The Local Regularization.}

The proof of the Main Theorem begins with the explicit construction of the approximate submanifolds $\bar{M}_{\alpha}$. These will be constructed by gluing an appropriate interpolation between $M_{1}$ and $M_{2}$ in a neighbourhood of small radius about the singular point of $M_{1} \cup M_{2}$. As mentioned in the Introduction, the interpolating submanifold that will be used is the special 
Lagrangian submanifold asymptotic to the tangent cone of $M$ at the singularity which is known as the Lawlor neck and exists whenever the planes comprising the tangent cone satisfy the angle criterion. The purpose of the present section is to describe the Lawlor submanifold. The actual gluing will be carried out in Section 3.2 and the relevant properties of the resulting submanifold $\bar{M}_{\alpha}$ will be derived in Section 3.3.

Without loss of generality, the singularity of $M$ is located at the origin in $\mathbf{R}^{2 n}$. Let $P_{1}$ and $P_{2}$ denote the tangent planes $T_{0} M_{1}$ and $T_{0} M_{2}$ respectively, and denote by $P$ the cone $P_{1} \cup P_{2}$ - this is the tangent cone of $M$ at 0 . The Lawlor neck is an embedded cylinder of the form $N=\Psi\left(\mathbf{R} \times \mathbf{S}^{n-1}\right)$, where $\Psi$ is a special Lagrangian embedding into $\mathbf{R}^{2 n}$, and the two ends of this embedding, namely $E_{1} \equiv \Psi\left((\lambda, \infty) \times \mathbf{S}^{n-1}\right)$ and $E_{2} \equiv \Psi\left((-\infty,-\lambda) \times \mathbf{S}^{n-1}\right)$ tend towards $P_{1}$ and $P_{2}$, respectively, in a pointwise sense as $\lambda \rightarrow \infty$ (i. e. $E_{1}$ can be written as a graph over $P_{1}$ outside a large enough ball, and the graphing function tends to zero as the radius of the ball increases). Because each rescaled submanifold $\varepsilon N$ is still special Lagrangian and asymptotic to $P$, the family of homotheties $\varepsilon N$ is a special Lagrangian regularization of the singular variety $P$. The idea behind the gluing construction of $\bar{M}_{\alpha}$ is to use a sufficiently small rescaling of $N$ as the interpolating submanifold.

The precise definition of the Lawlor neck proceeds as follows. First, let $a_{1}, \ldots, a_{n}$ be positive real numbers and let $P: \mathbf{R}^{n} \times \mathbf{R} \longrightarrow \mathbf{R}$ be the function given by

$$
P(a, \lambda) \equiv \frac{\left(1+a_{1} \lambda^{2}\right) \cdots\left(1+a_{n} \lambda^{2}\right)-1}{\lambda^{2}}
$$

Next, set

$$
\theta_{k}(a, \lambda) \equiv \int_{0}^{\lambda} \frac{-\mathrm{d} s}{\left(\frac{1}{a_{k}}+s^{2}\right) \sqrt{P(a, s)}}
$$

where $a=\left(a_{1}, \ldots, a_{n}\right)$. It is easy to see that the integrals (10) converge as $\lambda \rightarrow \pm \infty$. Let $\theta_{k}(a)$ denote the asymptotic values $\lim _{\lambda \rightarrow \infty} \theta_{k}(a, \lambda)$; then $\lim _{\lambda \rightarrow-\infty} \theta_{k}(a, \lambda)=-\theta_{k}(a)$. This terminology sets the stage for the definition of the Lawlor embeddings.

Definition 5. For every $a \in \mathbf{R}^{n}$ with $a_{k}>0$ for all $k$, the map $\Psi_{a}$ : $\mathbf{R} \times \mathbf{S}^{n-1} \longrightarrow \mathbf{R}^{2 n}$ defines a Lawlor neck $N_{a}=\Psi_{a}\left(\mathbf{R} \times \mathbf{S}^{n-1}\right)$ according to the following prescription. Let

$$
\Psi_{a}\left(\lambda, \mu^{1}, \ldots, \mu^{n}\right) \equiv\left(x^{1}(\lambda, \mu), \ldots, x^{n}(\lambda, \mu) ; y^{1}(\lambda, \mu), \ldots, y^{n}(\lambda, \mu)\right)
$$


where

$$
\begin{aligned}
& x^{k}(\lambda, \mu)=\mu^{k} \sqrt{\frac{1}{a_{k}}+\lambda^{2}} \cos \left(\frac{\pi}{2} \delta_{1 k}+\theta_{k}(a, \lambda)\right) \\
& y^{k}(\lambda, \mu)=\mu^{k} \sqrt{\frac{1}{a_{k}}+\lambda^{2}} \sin \left(\frac{\pi}{2} \delta_{1 k}+\theta_{k}(a, \lambda)\right) .
\end{aligned}
$$

and $\mu=\left(\mu^{1}, \ldots, \mu^{n}\right) \in \mathbf{R}^{n}$ satisfies $\sum\left(\mu^{k}\right)^{2}=1$ and thus $\mu$ represents a point in $\mathbf{S}^{n-1}$. Here, $\delta_{1 k}$ is the Kronecker symbol, defined to equal zero unless $k=1$, in which case it equals 1 .

It will be necessary to have precise numerical estimates of the degree of closeness between $N_{a}$ and $P_{1} \cup P_{2}$, but suitable coordinates must be found to perform the calculations. It is true that sufficiently far from the origin, the nearest point projection of $N_{a}$ onto one or the other of its asymptotic planes will be a diffeomorphism. Consequently, coordinates can be chosen so that the ends of $N_{a}$ are graphs over the corresponding asymptotic planes. The desired estimates on the asymptotics of the Lawlor neck $N_{a}$ will be phrased in terms of these graphing functions.

Theorem 6. Suppose $n \geq 3$. There exists a positive, real number $R_{0}$ so that $N_{a} \cap\left(B_{R_{0}}(0)\right)^{c}$ consists of two ends $E_{1}$ and $E_{2}$ that are graphs over $P_{1} \cap\left(B_{R_{0}}(0)\right)^{c}$ and $P_{2} \cap\left(B_{R_{0}}(0)\right)^{c}$, respectively, of the gradient of a single function $g: P_{i} \cap\left(B_{R_{0}}(0)\right)^{c} \longrightarrow P_{i}^{\perp}$. Furthermore, the function $g$ has the property that there exists some constant $C_{0}$ depending only on $a_{1}, \ldots, a_{n}$ and $n$ so that

$$
\|\nabla g(p)\|+\|p\|\|\operatorname{Hess} g(p)\|+\|p\|^{2}\left\|\nabla^{3} g(p)\right\| \leq \frac{C_{0}}{\|p\|^{n-1}}
$$

for any $p \in P_{i}$ with $\|p\| \geq R_{0}$; and the $\beta$-Hölder coefficient of $\nabla^{3} g$ satisfies

$$
\left[\nabla^{3} g\right]_{\beta,\left(B_{R}(0)\right)^{c}} \leq \frac{C_{0}}{R^{n+1+\beta}}
$$

for any radius $R \geq R_{0}$; and finally, $g$ can be chosen so that

$$
|g(p)| \leq \frac{C_{0}}{\|p\|^{n-2}}
$$

for any $p \in P_{i}$ with $\|p\| \geq R_{0}$. The norms and derivatives used here are those associated with the standard Euclidean metric on the planes $P_{i}$. 
Proof. Only the end $E_{1}$, asymptotic to the plane $P_{1}$ and corresponding to large positive $\lambda$, needs to be developed in detail since the calculations for $E_{2}$ are identical to those of $E_{1}$. Begin with a series of preliminary estimates.

Let $A=\min \left\{a_{1}, \ldots, a_{n}\right\}$ and put $R_{0}=\sqrt{2 / A}$. First, a calculation reveals that

$$
|P(a, \lambda)| \geq \min \left\{A^{n} \lambda^{2 n-2}, n A\right\} .
$$

Thus one can estimate

$$
\left|\theta_{k}(a, \lambda)-\theta_{k}(a)\right|=\int_{\lambda}^{\infty} \frac{\mathrm{d} s}{\left(\frac{1}{a_{k}}+s^{2}\right) \sqrt{P(a, s)}} \leq \frac{1}{n(\sqrt{A})^{n}} \cdot \frac{1}{\lambda^{n}} .
$$

Finally, a relation between $\lambda$ and $\|p\|$ for $p \in E_{1}$ can be found from the equation $\|p\|=\left\|\Psi_{a}(\lambda, \mu)\right\|=\sum\left(\left(x^{k}(\lambda, \mu)\right)^{2}+\left(y^{k}(\lambda, \mu)\right)^{2}\right)$ and the fact that $\sum\left(\mu^{k}\right)^{2}=1$. A simple calculation shows that when $\|p\| \geq R_{0}$, then

$$
\frac{\lambda}{\sqrt{2}} \leq\|p\| \leq \sqrt{2} \lambda
$$

In order to study the asymptotics of $E_{1}$ to $P_{1}$, it is most convenient to choose new coordinates in which $P_{1}$ is transformed into $R^{n} \times\{0\}$. For simplicity, let $\theta_{k}$ denote the angles $\frac{\pi}{2} \delta_{1 k}+\theta_{k}(a)$ and choose new coordinates $(s, t)$ for $\mathbf{R}^{2 n}$ according to the formulae:

$$
\begin{aligned}
& s^{k}=x^{k} \cos \left(\theta_{k}\right)+y^{k} \sin \left(\theta_{k}\right) \\
& t^{k}=-x^{k} \sin \left(\theta_{k}\right)+y^{k} \cos \left(\theta_{k}\right) .
\end{aligned}
$$

The coordinate functions (11b) of the embedding of the Lawlor neck are given in the new coordinates by the equations:

$$
\begin{aligned}
& s^{k}=\mu^{k} \sqrt{\frac{1}{a_{k}}+\lambda^{2}} \cos \left(\theta_{k}(a, \lambda)-\theta_{k}(a)\right) \\
& t^{k}=\mu^{k} \sqrt{\frac{1}{a_{k}}+\lambda^{2}} \sin \left(\theta_{k}(a, \lambda)-\theta_{k}(a)\right)
\end{aligned}
$$

where $(\lambda, \mu) \in \mathbf{R} \times \mathbf{S}^{n-1}$ and $\sum\left(\mu^{k}\right)^{2}=1$.

The Lawlor embedding given in (15) converges to the standard polar coordinate embedding of the plane $P_{1}$, that is to the embedding given by

$$
\begin{aligned}
& s^{k}=\mu^{k} \lambda \\
& t^{k}=0,
\end{aligned}
$$

where, again $\sum \mu_{k}^{2}=1$. Thus it can be shown that $E_{1}$ is a graph over $P_{1}$ outside a sufficiently large ball - and in fact that a ball of radius $R_{0}$ suffices. 
The coordinates $t^{k}$ restricted to the Lawlor neck can thus be written as functions of $s^{k}$ in the region $\|s\| \geq R_{0}$. Since the Lawlor neck is Lagrangian, it is the graph of the gradient of a function $g: \mathbf{R}^{n} \cap\left(B_{R_{0}}(0)\right)^{c} \longrightarrow \mathbf{R}$ over the asymptotic plane in this region. Thus $t^{k}=\frac{\partial g}{\partial s^{k}}$ are the partial derivatives of the function $g$.

The norm of the gradient of $g$ can now be estimated in the asymptotic region. Divide the first of equations (15) by the second. This gives the relation

$$
\frac{\partial g}{\partial s^{k}} \equiv t^{k}=s^{k} \tan \left(\theta_{k}(a, \lambda)-\theta_{k}(a)\right) \text {. }
$$

Suppose $\|s\| \geq R_{0}$. Now use the preliminary estimates (12) to (14) to estimate:

$$
\left|\frac{\partial g}{\partial s^{k}}\right| \leq \frac{2}{n}\left(\frac{2}{A}\right)^{n / 2} \frac{1}{\|s\|^{n-1}} .
$$

Consequently,

$$
\|\nabla g\|=\left(\sum_{k}\left|\frac{\partial g}{\partial s^{k}}\right|^{2}\right)^{1 / 2} \leq \frac{C_{0}}{\|s\|^{n-1}}
$$

where $C_{0}$ is some constant depending only on $n$ and $A$.

The bounds on the higher derivatives of $g$ come from differentiating equation (16) and estimating all quantities that appear using the preliminary estimates once again. Furthermore, it is possible to derive the estimate $\left\|\nabla^{4} g\right\| \leq C_{0}\|p\|^{-n-2}$ using similar calculations and the estimate on the Hölder coefficient of the third derivatives of $g$ follows from this in the standard way. Finally, the function $g$ itself is defined only up to an arbitrary constant; thus it is possible to choose

$$
g(s)=-\lim _{r \rightarrow \infty} \int_{\gamma(s, r s)} \mathrm{d} g,
$$

where $\gamma(s, r s)$ is the line segment between $s$ and $r s$. This integral is well defined because the derivatives of $g$ decay sufficiently rapidly near infinity. The bound on the size of $g$ now follows from the bound on the gradient of $g$ when $\|s\|$ is sufficiently large.

\subsection{Construction of $\bar{M}_{\alpha}$.}

The approximate submanifold $\bar{M}_{\alpha}$ will be constructed by removing a neighbourhood of the singularity of $M$ and smoothly reconnecting the pieces by a rescaling $\varepsilon N_{a}$ of the appropriate Lawlor neck $N_{a}$. The graphing functions 
of $N_{a}$ and $M$ over the tangent cone $P_{1} \cup P_{2}$ of $M$ at the singularity will be used to formulate a numerically precise version of this construction. Let $\pi_{i}$ be the orthogonal projection onto the plane $P_{i}$ and denote by $A n n_{\delta}$ the annulus $\left(B_{\delta / 2}(0)\right)^{c} \cap B_{\delta}(0)$. The graphical property of $M$ over its tangent cone can now be phrased in the following way.

Proposition 7. There is a constant $K$ and a number $\delta_{0}>0$ which depend only on the geometry of $M$ such that the following is true. There is a function $f_{i}: P_{i} \cap A n n_{\delta} \longrightarrow \mathbf{R}$ such that if $\delta \leq \delta_{0}$, then $M_{i} \cap \pi_{i}^{-1}\left(P_{i} \cap A n n_{\delta}\right)$ is the graph of $\nabla f_{i}$ over $P_{i} \cap A n n_{\delta}$. In addition, the function $f_{i}$ satisfies

$$
\frac{\partial^{2} f_{i}}{\partial x^{k} \partial x^{l}}(0)=\frac{\partial f_{i}}{\partial x^{k}}(0)=f_{i}(0)=0
$$

for all $k$ and $l$, along with the bounds:

$$
\begin{aligned}
& \left|f_{i}\right|_{0, B_{\delta}(0)}+\delta\left\|\nabla f_{i}\right\|_{0, B_{\delta}(0)}+\delta^{2}\left\|\nabla^{2} f_{i}\right\|_{0, B_{\delta}(0)} \\
& \quad+\delta^{3}\left\|\nabla^{3} f_{i}\right\|_{0, B_{\delta}(0)}+\delta^{3+\beta}\left[\nabla^{3} f_{i}\right]_{\beta, B_{\delta}(0)} \leq K \delta^{3}
\end{aligned}
$$

The norms and derivatives are those associated to the standard Euclidean metric on the planes $P_{i}$.

Proof. There is some neighbourhood of the origin in which each $M_{i}$ is graphical over its tangent plane; and in this neighbourhood, the extrinsic curvature of the submanifolds $M_{1}$ and $M_{2}$ is bounded in the $C^{0, \beta}$ norm by some number because of compactness. For a gradient graph, the extrinsic curvature is expressed in terms of the derivatives up to order three of the graphing function. The curvature condition thus translates into the bound

$$
\left\|\nabla^{3} f_{i}\right\|_{0, B_{\delta}(0)}+\delta^{\beta}\left[\nabla^{3} f_{i}\right]_{\beta, B_{\delta}(0)} \leq K
$$

on the graphing functions $f_{i}$. The estimates on the second and lower derivatives follow by integration, and use (18).

Analogous estimates can be found for the graphing function of $\varepsilon N_{a}$ over the components of $P$. Recall that $N_{a}$ is the graph of the gradient of some function $g$ outside the ball of radius $R_{0}$.

Proposition 8. Each asymptotic end $\varepsilon N_{a} \cap \pi_{i}^{-1}\left(P_{i} \cap\left(B_{\varepsilon R_{0}}(0)\right)\right)^{c}$ of the rescaled Lawlor neck is the graph of the gradient of a function $g_{\varepsilon}: P_{i} \cap$ $\left(B_{\varepsilon R_{0}}(0)\right)^{c} \longrightarrow \mathbf{R}$ over the appropriate asymptotic plane $P_{i}$. Moreover,

$$
g_{\varepsilon}(x)=\varepsilon^{2} g\left(\frac{x}{\varepsilon}\right) .
$$


Thus the function $g_{\varepsilon}$ satisfies the asymptotic inequalities

$$
\left|g_{\varepsilon}(x)\right|+\|x\|\left\|\nabla g_{\varepsilon}(x)\right\|+\|x\|^{2}\left\|\nabla^{2} g_{\varepsilon}(x)\right\|+\|x\|^{3}\left\|\nabla^{3} g_{\varepsilon}(x)\right\| \leq \frac{C_{0} \varepsilon^{n}}{\|x\|^{n-2}}
$$

for $x \in P_{i}$ with $\|x\| \geq \varepsilon R_{0}$, as well as the estimate

$$
\left[\nabla^{3} g_{\varepsilon}\right]_{\beta,\left(B_{R}(0)\right)^{c}} \leq \frac{C_{0} \varepsilon^{n}}{R^{n+1+\beta}}
$$

for any $R \geq \varepsilon R_{0}$. Here, both $R_{0}$ and $C_{0}$ are as in Theorem 6 and the norms and derivatives are those associated to the standard Euclidean metric on the planes $P_{i}$.

Proof. The identity $\nabla g_{\varepsilon}(x)=\varepsilon \nabla g\left(\frac{x}{\varepsilon}\right)$ for points on $P$ outside the ball of radius $\varepsilon R_{0}$ follows from scaling arguments. Consequently, $g_{\varepsilon}(x)=\varepsilon^{2} g\left(\frac{x}{\varepsilon}\right)$ (once the constant of integration is set to zero), and

$$
\left|g_{\varepsilon}(x)\right|=\varepsilon^{2}\left|g\left(\frac{x}{\varepsilon}\right)\right| \leq \frac{C_{0} \varepsilon^{n}}{\|x\|^{n-2}} .
$$

The remaining estimates follow from those of Theorem 6 in a similar way.

In order to ensure that both $M$ and the correct rescaling $\varepsilon N_{a}$ of the Lawlor neck are close to the tangent cone $P$ in the annulus $A n n_{\delta}$, the numbers $\varepsilon$ and $\delta$ will be chosen to produce

$$
\left\|\nabla^{2} f_{i}(x)\right\| \leq \alpha \quad \text { and } \quad\left\|\nabla^{2} g_{\varepsilon}(x)\right\| \leq \alpha
$$

for any $x \in P$ with $\frac{\delta}{2} \leq\|x\| \leq \delta$, where $\alpha$ is any sufficiently small, positive number.

Proposition 9. There is a number $A_{1}>0$ and a constant $C$ depending only on the geometry of $M$ and $N_{a}$ such that if $0<\alpha \leq A_{1}$ and the values

$$
\delta=\frac{\alpha}{K} \quad \text { and } \quad \varepsilon \leq C \alpha^{1+1 / n}
$$

are chosen, then $M_{i} \cap \pi_{i}^{-1}\left(P_{i} \cap A n n_{\delta}\right)$ for $i=1$ and 2, and $\varepsilon N_{a} \cap$ $\pi_{i}^{-1}\left(P_{i} \cap A n n_{\delta}\right)$ are graphs of $\nabla f_{i}$ and $\nabla g_{\varepsilon}$, respectively, over the annulus $P_{i} \cap A n n_{\delta}$. In addition, the norms of the Hessians of these functions are bounded above by $\alpha$; that is

$$
\left\|\nabla^{2} f_{i}(x)\right\| \leq \alpha \quad \text { and } \quad\left\|\nabla^{2} g_{\varepsilon}(x)\right\| \leq \alpha
$$

for any $x \in P_{i}$ satisfying $\frac{\delta}{2} \leq\|x\| \leq \delta$. 
Proof. By Proposition 7, it is sufficient to choose $\delta=\frac{\alpha}{K}$ in order to control the Hessian of each $f_{i}$. To achieve the second inequality, choose $\varepsilon$ small enough to bring the asymptotic region of $\varepsilon N_{a}$ into the annulus $A n n_{\delta}$. Thus

$$
\frac{\alpha}{2 K} \geq \varepsilon R_{0}
$$

is needed. Now the bounds of Proposition 8 are valid and thus for $x \in P$ with $\|x\| \geq \frac{\alpha}{2 K}$,

$$
\left\|\nabla^{2} g_{\varepsilon}(x)\right\| \leq \frac{C_{0} \varepsilon^{n}}{\|x\|^{n}} \leq 2^{n} K^{n} C_{0} \frac{\varepsilon^{n}}{\alpha^{n}}
$$

If $\varepsilon \leq \frac{\alpha^{1+1 / n}}{2 K \sqrt[n]{C_{0}}}$, then $\left\|\nabla^{2} g_{\varepsilon}(x)\right\| \leq \alpha$, as desired. If $\alpha$ is sufficiently small to begin with, then both choices of $\varepsilon$ can be made simultaneously.

The estimates of Proposition 8 as well as the bounds on the functions $f_{i}$ from equation (19) can now be reformulated in terms of the parameter $\alpha$.

Corollary 10. The functions $f_{i}$ and the function $g_{\varepsilon}$ from Proposition 9 also satisfy the bounds:

$$
\left[\nabla^{3} f_{i}\right]_{\beta, P_{i} \cap A n n_{\delta}} \leq K^{1+\beta} \alpha^{-\beta} \text { and }\left[\nabla^{3} g_{\varepsilon}\right]_{\beta, P_{i} \cap A n n_{\delta}} \leq(2 K)^{1+\beta} \alpha^{-\beta}
$$

along with

$$
\begin{aligned}
& \left\|\nabla^{3} f_{i}(x)\right\| \leq K \quad\left\|\nabla^{3} g_{\varepsilon}(x)\right\| \leq 2 K \\
& \left\|\nabla f_{i}(x)\right\| \leq \frac{\alpha^{2}}{K} \quad \text { and } \quad\left\|\nabla g_{\varepsilon}(x)\right\| \leq \frac{\alpha^{2}}{2 K} \\
& \left|f_{i}(x)\right| \leq \frac{\alpha^{3}}{K^{2}} \quad\left|g_{\varepsilon}(x)\right| \leq \frac{\alpha^{3}}{4 K^{2}}
\end{aligned}
$$

for any $x \in P_{i}$ satisfying $\frac{\delta}{2} \leq\|x\| \leq \delta$.

Proof. These estimates can be verified by substituting for $\delta$ and $\varepsilon$ in the appropriate equations (19) or (20) and by using $\frac{\delta}{2} \leq\|x\| \leq \delta$.

Suppose now that $\alpha \leq A_{1}$ and the quantities $\delta$ and $\varepsilon$ have been chosen according to Proposition 9. It remains to be seen how to glue $M$ and $\varepsilon N_{a}$ together in the annulus $A n n_{\delta}$ in order to build the smooth submanifold $\bar{M}_{\alpha}$. The 'stickiness' is provided by a cut-off function: let $\eta: \mathbf{R}^{2 n} \rightarrow \mathbf{R}$ denote a positive, $C^{\infty}$ function which is equal to zero outside the ball of radius $\delta$, one 
inside the ball of radius $\frac{\delta}{2}$ and interpolates smoothly in between such that the supremum norm bounds (for the Euclidean metric on $\mathbf{R}^{2 n}$ )

$$
|\eta|_{0, \mathbf{R}^{2 n}}+\delta\|\nabla \eta\|_{0, \mathbf{R}^{2 n}}+\delta^{2}\left\|\nabla^{2} \eta\right\|_{0, \mathbf{R}^{2 n}}+\delta^{3}\left\|\nabla^{3} \eta\right\|_{0, \mathbf{R}^{2 n}} \leq C
$$

hold in the annulus $A n n_{\delta}$, where $C$ is some geometric constant depending only on $n$. The approximate submanifold $\bar{M}_{\alpha}$ is the union of five pieces that overlap smoothly thanks to the cut-off function $\eta$.

Definition 11. Suppose $\alpha \leq A_{1}$ and $\varepsilon$ and $\delta$ have been chosen as in Proposition 9. Define the following submanifolds.

$$
\begin{array}{ll}
\text { 1) } & M_{i}^{\prime}=M_{i} \backslash \pi_{i}^{-1}\left(P_{i} \cap B_{\delta}(0)\right) \quad \text { for } i=1,2 \\
\text { 2) } & T_{i}=\left\{\left(x, \nabla\left((1-\eta) f_{i}+\eta g_{\varepsilon}\right)(x)\right) \in P_{i} \times P_{i}^{\perp}: \frac{\delta}{2} \leq\|x\| \leq \delta\right\} \quad \text { for } i=1,2 \\
\text { 3) } & N^{\prime}=\varepsilon N_{a} \cap\left(\pi_{2}^{-1}\left(P_{2} \cap B_{\frac{\delta}{2}}(0)\right) \cup \pi_{2}^{-1}\left(P_{2} \cap B_{\frac{\delta}{2}}(0)\right)\right)
\end{array}
$$

The approximate solution to the deformation problem is the submanifold

$$
\bar{M}_{\alpha}=M_{1}^{\prime} \cup T_{1} \cup N^{\prime} \cup T_{2} \cup M_{2}^{\prime} .
$$

The submanifold $M_{1}^{\prime} \cup M_{2}^{\prime}$ is called the exterior region of $\bar{M}_{\alpha}$, the submanifold $T_{1} \cup T_{2}$ is called the transition region of $\bar{M}_{\alpha}$ and the submanifold $T_{1} \cup N^{\prime} \cup T_{2}$ is called the neck region of $\bar{M}_{\alpha}$.

The following theorem shows that the $\bar{M}_{\alpha}$ are indeed smooth Lagrangian approximations of $M$.

Theorem 12. The submanifolds $\bar{M}_{\alpha}$, with $\alpha \leq A_{1}$, that have been constructed above are smooth, Lagrangian submanifolds of $\mathbf{R}^{2 n}$ which converge to the singular submanifold $M$ in a pointwise sense as $\alpha \rightarrow 0$.

Proof. The submanifold $\bar{M}_{\alpha}$ is smooth because each $T_{i}$ overlaps smoothly with its neighbours as a result of the way in which the graphing functions $f_{i}$ and $g_{\varepsilon}$ were combined.

Furthermore, both $M$ and $\varepsilon N_{\alpha}$ are Lagrangian and thus $\bar{M}_{\alpha} \backslash\left(T_{1} \cup\right.$ $\left.T_{2}\right)$ is Lagrangian. But the transition regions $T_{i}$ are gradient graphs over Lagrangian planes in the symplectic coordinates given by the splitting $P_{i} \times$ $P_{i}^{\perp}$ and thus are Lagrangian as well.

Convergence is easily verified. The distance between $M$ and $\bar{M}_{\alpha}$ is actually zero outside a neighbourhood of radius on the order of $\alpha$. Since this neighbourhood is shrinking and $M_{\alpha}$ is smooth, $\bar{M}_{\alpha} \rightarrow M$ as $\alpha \rightarrow 0$. 


\subsection{Properties of $\bar{M}_{\alpha}$.}

The remainder of this section is devoted to deriving the properties of $\bar{M}_{\alpha}$ that will be used in the sequel. The first such property concerns how well $\bar{M}_{\alpha}$ approximates a special Lagrangian submanifold near $M$. The upcoming estimates will be explicitly of a global nature, unlike the previous estimates, so they must be phrased using the induced metric of $\bar{M}_{\alpha}$, and will thus make use of the following bounds on the metric components and their derivatives.

Lemma 13. Let $g_{k l}^{i}$ denote the coefficients of the induced metric in the graphical coordinates for the transition region $T_{i}$. There is a number $A_{g}>0$ so that if $\alpha \leq A_{g}$ then the functions $g_{k l}^{i}$ satisfy

$$
\left\|\left(g_{k l}^{i}(x)\right)-I\right\| \leq 1 \quad \text { and } \quad \frac{1}{2} \leq\left|\operatorname{det}\left(g_{k l}^{i}(x)\right)\right| \leq 2
$$

along with the derivative bounds

$$
\left|\frac{\partial g_{k l}^{i}}{\partial x^{m}}\right|_{0, A n n_{\delta}}+\delta^{\beta}\left[\frac{\partial g_{k l}^{i}}{\partial x^{m}}\right]_{\beta, A n n_{\delta}} \leq 1
$$

in the annulus $P_{i} \cap A n n_{\delta}$.

Proof. This is a straightforward calculation using the expression for the metric of the transition region in the graphical coordinates.

It is now possible to measure the extent to which $\bar{M}_{\alpha}$ deviates from being special Lagrangian in a manner independent of coordinates. Of course, by definition, $\bar{M}_{\alpha}$ is exactly special Lagrangian outside the transition region.

Proposition 14. Let $\bar{M}_{\alpha}$ be the submanifold constructed in Theorem 12 and suppose that $\bar{h}_{\alpha}$ embeds this submanifold into $\mathbf{R}^{2 n}$. There exist a constant $C$ and a number $A_{2}$ with $0<A_{2} \leq \min \left\{A_{1}, A_{g}\right\}$, both independent of $\alpha$, such that whenever $\alpha \leq A_{2}$, the pull back of the form $\mathrm{d} z$ satisfies the estimates

$$
\begin{gathered}
\left\|\bar{h}_{\alpha}^{*}(\operatorname{Im} \mathrm{d} z)\right\|_{0, T_{1} \cup T_{2}} \leq C \alpha \\
1-C^{2} \alpha^{2} \leq\left\|\bar{h}_{\alpha}^{*}(\operatorname{Re} \mathrm{d} z)\right\|_{0, T_{1} \cup T_{2}}^{2} \leq 1
\end{gathered}
$$

and

$$
\begin{aligned}
& \left\|\nabla \bar{h}_{\alpha}^{*}(\operatorname{Im} \mathrm{d} z)\right\|_{0, T_{1} \cup T_{2}}+\alpha^{\beta}\left[\nabla \bar{h}_{\alpha}^{*}(\operatorname{Im} \mathrm{d} z)\right]_{\beta, T_{1} \cup T_{2}} \leq C \\
& \left\|\nabla \bar{h}_{\alpha}^{*}(\boldsymbol{\operatorname { R e }} \mathrm{d} z)\right\|_{0, T_{1} \cup T_{2}}+\alpha^{\beta}\left[\nabla \bar{h}_{\alpha}^{*}(\boldsymbol{\operatorname { R e }} \mathrm{d} z)\right]_{\beta, T_{1} \cup T_{2}} \leq C
\end{aligned}
$$


within the transition region.

Proof. Only the estimates for $T_{1}$ need to be performed since the computations for $T_{2}$ are identical. Choose coordinates so that $T_{1}$ is the graph of the Euclidean gradient of $(1-\eta) f_{1}+\eta g_{\varepsilon}$ over the annular region $A n n_{\delta}$ in $\mathbf{R}^{n} \times\{0\}$. The operators $\nabla^{2}$ and $\nabla$ in the following calculations refer to the derivatives associated to the Euclidean metric in this coordinate system.

Begin with the bound on the size of $\bar{h}_{\alpha}^{*}(\mathbf{I m} \mathrm{d} z)$ in the transition region. In their paper, Harvey and Lawson compute the pull back of the form $\mathbf{I m} \mathrm{d} z$ to a graphical Lagrangian submanifold of $\mathbf{R}^{2 n}[6]$. What they obtain is the expression

$$
\bar{h}_{\alpha}^{*}(\mathbf{I m} \mathrm{d} z)=\mathbf{I m}\left[\operatorname{det}_{\mathbf{C}}\left(I+\mathrm{i} \nabla^{2}\left((1-\eta) f_{1}+\eta g_{\varepsilon}\right)\right)\right] \mathrm{d} x^{1} \wedge \cdots \wedge \mathrm{d} x^{n} .
$$

By the Taylor expansion of the function $t \mapsto \operatorname{det}_{\mathbf{C}}(I+\mathrm{i} t A)$, there exists a number $r$ and of a constant $C$ such that if $\|A\| \leq r$, then $\left|\operatorname{Im} \operatorname{det}_{\mathbf{C}}(I+\mathrm{i} A)\right| \leq$ $C\|A\|$. Given the bounds on the functions $\eta, g_{\varepsilon}$ and $f_{1}$ and their derivatives, it is clear that there is a number $A_{2}$ (which should be chosen smaller than $A_{1}$ and $A_{g}$ ) so that if $\alpha \leq A_{2}$, then

$$
\begin{aligned}
\mid \operatorname{Im}_{\operatorname{det}} \mathbf{C}\left(I+\mathrm{i} \nabla^{2}((1-\eta)\right. & \left.\left.f_{1}+\eta g_{\varepsilon}\right)\right)\left.\right|_{0, \mathbf{R}^{n}} \leq C\left|\nabla^{2}\left((1-\eta) f_{1}+\eta g_{\varepsilon}\right)\right|_{0, \mathbf{R}^{n}} \\
\leq & \quad|1-\eta|\left\|\nabla^{2} f_{i}\right\|+|\eta|\left\|\nabla^{2} g_{\varepsilon}\right\|+\left\|\nabla^{2} \eta\right\|\left|f_{i}+g_{\varepsilon}\right| \\
& \quad+2\|\nabla \eta\|\left(\left\|\nabla f_{i}\right\|+\left\|\nabla g_{\varepsilon}\right\|\right) \\
\leq & 1 \cdot \alpha+1 \cdot \alpha+\frac{C n}{\delta^{2}} \cdot \frac{2 \alpha^{3}}{K^{2}}+2 \cdot \frac{C}{\delta} \cdot \frac{2 \alpha^{2}}{K} \\
& =C \alpha
\end{aligned}
$$

where the constant $C$ depends only on $A_{2}$ and $n$. The fact that $\delta=\frac{\alpha}{K}$ has been used here.

Equation (27) is an estimate for the desired quantity in the Euclidean norm. This must now be converted to a global estimate. Let $g_{\bar{M}_{\alpha}}$ denote the induced metric on $\bar{M}_{\alpha}$. Then,

$$
\begin{aligned}
& \left\|\bar{h}_{\alpha}^{*}(\operatorname{Im} \mathrm{d} z)\right\|_{0, T_{1} \cup T_{2}} \\
& =\left|\operatorname{Im} \operatorname{det}_{\mathbf{C}}\left(I+\mathrm{i} \nabla^{2}\left((1-\eta) f_{1}+\eta g_{\varepsilon}\right)\right)\right|_{0, \mathbf{R}^{n}}\left\|\mathrm{~d} x^{1} \wedge \cdots \wedge \mathrm{d} x^{n}\right\|_{0, T_{1} \cup T_{2}} \\
& \leq \frac{C \alpha}{\left(\operatorname{det}\left(g_{\bar{M}_{\alpha}}\right)\right)^{1 / 2}} .
\end{aligned}
$$


For the desired estimate, invoke Lemma 13 to bound the metric term in this expression from below.

The covariant derivative of $\operatorname{Im} \mathrm{d} z$ also only needs to be analyzed in $T_{1}$ and once again, the local, graphical coordinates can be used for this purpose. The calculations are similar to the ones above, though far more tedious because they involve the third derivatives of $\eta$ and of the graphing functions along with their Hölder coefficients. Finally, the bounds on $\bar{h}_{\alpha}^{*}(\boldsymbol{R e} \mathrm{d} z)$ follow in the same way from the identity $\|\mathrm{d} z\|=1$ proved in Harvey and Lawson's paper [6].

REMARK: The quantities $\bar{h}_{\alpha}^{*}(\operatorname{Im} \mathrm{d} z)$ and $\bar{h}_{\alpha}^{*}(\boldsymbol{\operatorname { R e }} \mathrm{d} z)$ estimated in the previous proposition can be related to the Lagrangian angle function of $\bar{M}_{\alpha}$. According to the defining equation (6) of this function,

$$
\begin{aligned}
& \sin \left(\theta_{\bar{M}_{\alpha}}\right)=\left\langle\bar{h}_{\alpha}^{*}(\operatorname{Im} \mathrm{d} z), \operatorname{Vol}_{\bar{M}_{\alpha}}\right\rangle \\
& \cos \left(\theta_{\bar{M}_{\alpha}}\right)=\left\langle\bar{h}_{\alpha}^{*}(\operatorname{Re} \mathrm{d} z), \operatorname{Vol}_{\bar{M}_{\alpha}}\right\rangle .
\end{aligned}
$$

Consequently, the trigonometric functions of $\theta_{\bar{M}_{\alpha}}$ satisfy the following estimates:

$$
\left|\sin \left(\theta_{\bar{M}_{\alpha}}\right)\right|_{0, T} \leq C \alpha \quad \text { and } \quad \sqrt{1-C^{2} \alpha^{2}} \leq\left|\cos \left(\theta_{\bar{M}_{\alpha}}\right)\right|_{0, T} \leq 1
$$

as well as

$$
\begin{array}{r}
\left|\nabla \sin \left(\theta_{\bar{M}_{\alpha}}\right)\right|_{0, T}+\alpha^{\beta}\left[\nabla \sin \left(\theta_{\bar{M}_{\alpha}}\right)\right]_{\beta, T} \leq C \\
\left|\nabla \cos \left(\theta_{\bar{M}_{\alpha}}\right)\right|_{0, T}+\alpha^{\beta}\left[\nabla \sin \left(\theta_{\bar{M}_{\alpha}}\right)\right]_{\beta, T} \leq C .
\end{array}
$$

These last two equations imply

$$
\left[\sin \left(\theta_{\bar{M}_{\alpha}}\right)\right]_{\beta, T} \leq C \alpha^{1-\beta} \quad \text { and } \quad\left[\cos \left(\theta_{\bar{M}_{\alpha}}\right)\right]_{\beta, T} \leq C \alpha^{1-\beta} .
$$

These estimates will be used repeatedly throughout the remainder of the paper.

The submanifold $\bar{M}_{\alpha}$ is almost special Lagrangian since the quantities $\operatorname{Im} \mathrm{d} z$ and $\boldsymbol{R e} \mathrm{d} z$ are close to their special Lagrangian values as $\alpha \rightarrow 0$. Since special Lagrangian submanifolds are minimal, the mean curvature vector of $\bar{M}_{\alpha}$ should thus also be controllable.

Proposition 15. Whenever $\alpha \leq A_{2}$, the mean curvature vector of $\bar{M}_{\alpha}$ satisfies the estimate

$$
\left\|\vec{H}_{\bar{M}_{\alpha}}\right\|_{0, T_{1} \cup T_{2}}+\alpha^{\beta}\left[\vec{H}_{\bar{M}_{\alpha}}\right]_{\beta, T_{1} \cup T_{2}} \leq C
$$

within the transition region, where $C$ is independent of $\alpha$. 
Proof. Recall that the mean curvature vector $\vec{H}_{\bar{M}_{\alpha}}$ of $\bar{M}_{\alpha}$ is related to the Lagrangian angle function by $\left.\bar{h}_{\alpha}^{*}\left(\vec{H}_{\bar{M}_{\alpha}}\right\rfloor \omega\right)=\mathrm{d} \theta_{\bar{M}_{\alpha}}$. In the transition region, the angle is non-constant and $\mathrm{d} \theta_{\bar{M}_{\alpha}}=\frac{\mathrm{d} \sin \left(\theta_{\bar{M}_{\alpha}}\right)}{\cos \left(\theta_{\bar{M}_{\alpha}}\right)}$. The estimates of (29) and calculations similar to those of Proposition 14 can be used to obtain

$$
\left\|\mathrm{d} \theta_{\bar{M}_{\alpha}}\right\|_{0, T_{1} \cup T_{2}}+\alpha^{\beta}\left[\mathrm{d} \theta_{\bar{M}_{\alpha}}\right]_{\beta, T_{1} \cup T_{2}} \leq C,
$$

where $C$ is independent of $\alpha$. The correspondence $\vec{H} \mapsto \vec{H}\rfloor \omega$ is an isometry, hence $\left\|\vec{H}_{\bar{M}_{\alpha}}\right\|_{0}+\alpha^{\beta}\left[\vec{H}_{\bar{M}_{\alpha}}\right]_{\beta}$ in the transition region is bounded by the same geometric constant given above.

In addition to these bounds on the form $\mathrm{d} z$ and the mean curvature $\vec{H}_{\bar{M}_{\alpha}}$, estimates on the volume of the neck region of $\bar{M}_{\alpha}$ will also be required in the proof of the Main Theorem. The volume will be calculated in two stages: first the volume of the transition region will be estimated and then the volume of the rescaled Lawlor neck itself will be estimated. Begin in the transition region $T_{1} \cup T_{2}$ by using the results of Lemma 13 .

Proposition 16. If $\alpha \leq A_{2}$, then the volume of the transition region satisfies $\operatorname{Vol}\left(T_{1} \cup T_{2}\right) \leq C \alpha^{n}$, where $C$ is a constant independent of $\alpha$.

Proof. The computation of the volume is best carried out in local coordinates in each component $T_{i}$. Let $g_{i j}$ denote the components of the induced metric $g_{\bar{M}_{\alpha}}$ in the local, graphical coordinates for $T_{1}$ (as usual, the results for the transition region $T_{2}$ are identical). Then,

$$
\begin{aligned}
\operatorname{Vol}\left(T_{1}\right) & =\int_{P_{1} \cap A n n_{\delta}} \sqrt{\operatorname{det}\left(g_{i j}\right)} \mathrm{d} x^{1} \wedge \cdots \wedge \mathrm{d} x^{n} \\
& \leq \sqrt{2} \int_{P_{1} \cap A n n_{\delta}} \mathrm{d} x^{1} \wedge \cdots \wedge \mathrm{d} x^{n}
\end{aligned}
$$

according to Lemma 13. Since $\delta=\frac{\alpha}{2 K}$, the last integral above is bounded by a number proportional to $\alpha^{n}$. Combining this fact with the inequality above (and adding the contribution to the volume from $T_{2}$ ) proves the proposition.

The upper bound on the volume of $N^{\prime}$, the remaining portion of the neck region of $\bar{M}_{\alpha}$, is slightly more subtle. The submanifold $N^{\prime}$ is a truncation of 
a scaled Lawlor neck; thus its volume exhibits the following scaling property:

$$
\begin{aligned}
\operatorname{Vol}\left(N^{\prime}\right) & =\operatorname{Vol}\left(\varepsilon N_{a} \cap\left(\pi_{1}^{-1}\left(P_{1} \cap B_{\frac{\delta}{2}}(0)\right) \cup \pi_{2}^{-1}\left(P_{2} \cap B_{\frac{\delta}{2}}(0)\right)\right)\right) \\
& =\varepsilon^{n} \operatorname{Vol}\left(N_{a} \cap\left(\pi_{1}^{-1}\left(P_{1} \cap B_{\frac{\delta}{2 \varepsilon}}(0)\right) \cup \pi_{2}^{-1}\left(P_{2} \cap B_{\frac{\delta}{2 \varepsilon}}(0)\right)\right)\right) .
\end{aligned}
$$

It is now fairly easy to use the definition of the Lawlor embeddings to conclude that

$$
N_{a} \cap\left(\pi_{1}^{-1}\left(B_{\frac{\delta}{2 \varepsilon}}(0)\right) \cup \pi_{2}^{-1}\left(B_{\frac{\delta}{2 \varepsilon}}(0)\right)\right) \subseteq \Psi_{a}\left(\left[-\frac{\delta}{\varepsilon}, \frac{\delta}{\varepsilon}\right] \times \mathbf{S}^{n-1}\right) .
$$

Recall that $\frac{\delta}{\varepsilon}=C \alpha^{-1 / n}$ where $C$ is independent of $\alpha$. The volume of $N^{\prime}$ can now be estimated in terms of the volume of this portion of the unscaled Lawlor neck $N_{a}$. But to accomplish this, more precise knowledge of the metric and the volume form on $N_{a}$ is needed.

Lemma 17. The volume element on $\mathbf{R} \times \mathbf{S}^{n-1}$ corresponding to the metric induced from $\mathbf{R}^{2 n}$ by the Lawlor embedding $\Psi_{a}$ satisfies the estimate

$$
\mathrm{Vol}_{N_{a}} \leq \mathrm{Vol}_{0}
$$

in the region $|\lambda| \geq A^{-1 / 2}$, where $\mathrm{Vol}_{0}$ is the volume form of the metric $g_{0}=2(\mathrm{~d} \lambda)^{2}+2 \lambda^{2} g_{S}$ on $\mathbf{R} \times S^{n-1}$ and $g_{S}$ is the standard metric on the unit sphere.

Proof. The metric on $\mathbf{R} \times \mathbf{S}^{n-1}$ induced by the Lawlor embedding is

$$
g_{N_{a}}=\left(\sum_{k=1}^{n} \frac{\left(\mu^{k}\right)^{2}}{a_{k}^{-1}+\lambda^{2}}\right)\left(\lambda^{2}+\frac{1}{P}\right)(\mathrm{d} \lambda)^{2}+\sum_{k=1}^{n}\left(a_{k}^{-1}+\lambda^{2}\right)\left(\mathrm{d} \mu^{k}\right)^{2}
$$

where the $\mu$ coordinates are restricted to the unit sphere $\sum\left(\mu^{k}\right)^{2}=1$ in $\mathbf{R}^{n}$ and $P=P(a, \lambda)$. Thus it is a straightforward calculation to estimate $\operatorname{Vol}_{N_{a}}$ in terms of $\mathrm{Vol}_{0}$.

The preceding lemma leads to the estimate on the volume of the neck region of $\bar{M}_{\alpha}$.

Proposition 18. There is a constant $C$ independent of $\alpha$ such that if $\alpha \leq$ $A_{2}$, then the volume of the neck region of $\bar{M}_{\alpha}$ satisfies $\operatorname{Vol}\left(T_{1} \cup N^{\prime} \cup T_{2}\right) \leq$ $C \alpha^{n}$. 
Proof. According to Lemma 17 and the scaling property (30) of the volume of the neck region of $\bar{M}_{\alpha}$, the following estimate is valid:

$$
\begin{aligned}
\operatorname{Vol}\left(T_{1} \cup N^{\prime} \cup T_{2}\right) & =\operatorname{Vol}\left(T_{1} \cup T_{2}\right)+\operatorname{Vol}\left(N^{\prime}\right) \\
& =C \alpha^{n}\left(1+\alpha \int_{\left[-C \alpha^{-1 / n}, C \alpha^{-1 / n}\right] \times \mathbf{S}^{n-1}} \operatorname{Vol}_{N_{a}}\right),
\end{aligned}
$$

where the result of Proposition 16 as well as the values of $\delta$ and $\varepsilon$ in terms of $\alpha$ have been used. According to equation (31), the volume form $\operatorname{Vol}_{N_{a}}$ can be replaced by the volume form $\operatorname{Vol}_{0}$ in the region where $|\lambda| \geq A^{-1 / 2}$. Consequently,

$$
\operatorname{Vol}\left(N^{\prime}\right) \leq C+C^{\prime} \int_{\left[A^{-1 / 2}, C \alpha^{-1 / n}\right] \times \mathbf{S}^{n-1}} \operatorname{Vol}_{0}=C+C^{\prime} \alpha^{-1}
$$

Combining the two results above and modifying the constants yields the desired estimate.

An obvious corollary to the volume bound on the neck region of $\bar{M}_{\alpha}$ is the following result.

Corollary 19. The volume of $\bar{M}_{\alpha}$ is uniformly bounded above and below whenever $\alpha \leq A_{2}$.

\subsection{The First Neumann Eigenvalue of $\bar{M}_{\alpha}$.}

The approximating submanifolds $\bar{M}_{\alpha}$ are converging to a singular variety as $\alpha \rightarrow 0$, which will have the effect of causing $\alpha$-dependent quantities on $\bar{M}_{\alpha}$ to degenerate as $\alpha \rightarrow 0$. The most important of these in the context of this paper is the first Neumann eigenvalue of the Laplacian on $\bar{M}_{\alpha}$, which tends towards zero as $\alpha \rightarrow 0$. The specific functional dependence of this quantity on $\alpha$ is a result of the geometry of $\bar{M}_{\alpha}$, and is established in the following proposition.

Proposition 20. The first Neumann eigenvalue of $\bar{M}_{\alpha}$ satisfies $\nu_{1} \leq$ $C_{1} \alpha^{n-2}$, where $C_{1}$ is a constant independent of $\alpha$.

Proof. Recall that the first Neumann eigenvalue of $\bar{M}_{\alpha}$ is equal to

$$
\nu_{1}=\inf \left\{\frac{\int_{\bar{M}_{\alpha}}\|\nabla u\|^{2}}{\int_{\bar{M}_{\alpha}} u^{2}}: u \in H^{1}\left(\bar{M}_{\alpha}\right) \text { and } \int_{\bar{M}_{\alpha}} u=0\right\}
$$


where $H^{1}\left(\bar{M}_{\alpha}\right)$ are the $L^{2}$ functions of $\bar{M}_{\alpha}$ whose first weak derivatives are also in $L^{2}$. Choose a function $u$ on $\bar{M}_{\alpha}$ which is equal to 1 in $M_{1}^{\prime}$, equal to -1 in $M_{2}^{\prime} \cap\left(\mathcal{U}_{\alpha}\right)^{c}$ and interpolates between these values in the neck region of $\bar{M}_{\alpha}$. The interpolation can thus be made in such a way that $|u| \leq 1$ and $\|\nabla u\| \leq C \alpha^{-1}$ for some constant $C$ independent of $\alpha$, and a constant can be subtracted to ensure that the function $u$ has integral zero. Now estimate as follows:

$$
\int_{\bar{M}_{\alpha}}\|\nabla u\|^{2}=\int_{\bar{M}_{\alpha} \cap \mathcal{U}_{\alpha}}\|\nabla u\|^{2} \leq C \alpha^{n-2},
$$

using the volume estimates of Proposition 18. Next, since $u$ differs from a constant outside a neighbourhood of size proportional to $\alpha$, the integral $\int_{\bar{M}_{\alpha}} u^{2}$ is bounded below by a constant independent of $\alpha$. Taking this fact together with (33) yields the desired estimate.

\section{Deformations of $\bar{M}_{\alpha}$.}

\subsection{Parametrizing Lagrangian Embeddings of $\bar{M}_{\alpha}$.}

The next step in the proof of the Main Theorem is to define the parametrization of Lagrangian embeddings of $\bar{M}_{\alpha}$ near $\bar{h}_{\alpha}$ that will be used to set up the PDE which must be solved using the Inverse Function Theorem. Unless the parametrization of Lagrangian submanifolds near $\bar{M}_{\alpha}$ is chosen with care, the strong dependence of $\nu_{1}$ on $\alpha$ will manifest itself in the dependence of $C_{L}(\alpha)$ on $\alpha$. For, suppose that the Banach space parametrizing nearby Lagrangian submanifolds contained the first Neumann eigenfunction of the Laplacian on $\bar{M}_{\alpha}$. Denote this function by $S_{\alpha}$. Then,

$$
\mathrm{D} F_{\alpha}(0,0)\left(S_{\alpha}, 0\right)=\nu_{1} \cos \left(\theta_{\bar{M}_{\alpha}}\right) S_{\alpha}-\sin \left(\theta_{\bar{M}_{\alpha}}\right)\left\langle\vec{H}_{\bar{M}_{\alpha}}, \nabla S_{\alpha}\right\rangle_{\bar{M}_{\alpha}},
$$

according to calculation of the linearization performed in Section 2.3, and indicates that the constant $C_{L}(\alpha)$ would be less than some quantity proportional to $\nu_{1}$. Thus the parametrizing Banach space should exclude $S_{\alpha}$ in order to achieve a better estimate of $C_{L}(\alpha)$.

With this observation in mind, begin by choosing a Banach space over which to parametrize embeddings. Let $Z$ be the inward pointing unit normal of $\partial \bar{M}_{\alpha}$.

Definition 21. Define the Banach space $\mathcal{B}_{\alpha}=\mathcal{B}_{1, \alpha} \times \mathbf{R}$ where

$$
\mathcal{B}_{1, \alpha}=\left\{H \in C^{2, \beta}\left(\bar{M}_{\alpha}\right):\left.Z(H)\right|_{\partial \bar{M}_{\alpha}}=0 \text { and } \int_{\bar{M}_{\alpha}} H=\int_{\bar{M}_{\alpha}} H \cdot S_{\alpha}=0\right\} .
$$


Here, $\beta \in(0,1)$ is the degree of Hölder continuity and will be chosen later.

REMARK: The constant functions (the kernel of the linearized operator) and the first eigenfunction $S_{\alpha}$ are excluded from $\mathcal{B}_{1, \alpha}$ because the integral conditions in the definition above ensure that the functions in $\mathcal{B}_{1, \alpha}$ are $L^{2}$ orthogonal to the constants and $S_{\alpha}$. The $\mathbf{R}$ factor in the definition above will be related to a deformation of $\bar{M}_{\alpha}$ taking $\partial \bar{M}_{\alpha}$ away from its scaffold $W$ and is will be used to guarantee the surjectivity of the linearized operator.

The parametrization of Lagrangian embeddings of $\bar{M}_{\alpha}$ near $\bar{h}_{\alpha}$ will be of the following form. To each $(H, b) \in \mathcal{B}_{1, \alpha} \times \mathbf{R}$, associate the embedding $\phi_{v_{e}}^{b} \circ \phi_{H_{e}}^{1} \circ \bar{h}_{\alpha}$, where the $\phi_{H_{e}}^{1}$ term is the time- 1 Hamiltonian flow of a suitable extension of the function $H$, and the $\phi_{v_{e}}^{b}$ term is the time- $b$ Hamiltonian flow of the extension of a carefully chosen function $v: \partial \bar{M}_{\alpha} \rightarrow \mathbf{R}$. In order to describe the extended functions $H_{e}$ and $v_{e}$ in greater detail, a lemma concerning the structure of tubular neighbourhoods of $\partial \bar{M}_{\alpha}$ is needed first.

Lemma 22. Let $W$ be a symplectic submanifold of codimension 2 in $\mathbf{R}^{2 n}$ and suppose that $L$ is a Lagrangian submanifold with boundary $\partial L \subset W$. Then there exists a tubular neighbourhood $\mathcal{U}$ of the boundary and a symplectomorphism $\psi: \mathcal{U} \longrightarrow\left(T^{*} \partial L\right) \times \mathbf{R}^{2}$ with the following properties:

1. $\psi(W \cap \mathcal{U}) \subset T^{*}(\partial L) \times\{0,0\}$;

2. $\psi(\partial L)=\partial L \times\{0,0\}$;

3. $\psi(L \cap \mathcal{U}) \subset \partial L \times \mathbf{R}_{+} \times\{0\}$; and

4. $\psi_{*} Z=\frac{\partial}{\partial s^{1}}$ along $\partial L$, where $\left(s^{1}, s^{2}\right)$ are the coordinates for the $\mathbf{R}^{2}$ factor and $Z$ is the inward unit normal of $\partial L$.

Proof. The proof of this lemma can be found in [2].

Definition of $H_{e}$

The function $H$ is extended in two stages: $H$ is first extended in an obvious manner to a neighbourhood of $\bar{M}_{\alpha}$; then this extension is modified near the boundary to ensure that the Hamiltonian deformation $\phi_{H_{e}}^{t}$ keeps $\partial \bar{M}_{\alpha}$ confined to $W$. The preliminary extension will be carried out in a tubular neighbourhood of $\bar{M}_{\alpha}$ chosen according to the following considerations.

Recall that the submanifold $\bar{M}_{\alpha}$ consists of two large pieces $M_{1}^{\prime}$ and $M_{2}^{\prime}$ which are connected by a thin neck. Hence there is a tubular neighbourhood 
for $\bar{M}_{\alpha}$ which is large around each $M_{i}^{\prime}$ but small in the vicinity of the neck. Denote this neighbourhood by $\mathcal{U}_{1}$. Without loss of generality, this tubular neighbourhood is symplectomorphic to a neighbourhood of the zero section in $T^{*} \bar{M}_{\alpha}$. Let $\tau: \mathbf{R}^{2 n} \longrightarrow \mathbf{R}$ be a smooth function equal to 1 in a tubular neighbourhood $\mathcal{U}_{1}^{\prime}$ of $\bar{M}_{\alpha}$ contained in $\mathcal{U}_{1}$ and that vanishes outside $\mathcal{U}_{1}$. Furthermore, suppose $\tau$ satisfies $|\tau|+\alpha\|\nabla \tau\| \leq C$ (the factor $\alpha$ arises because of the narrowness $\mathcal{U}_{1}$ near the neck region of $\bar{M}_{\alpha}$ ). Now define $H_{1}: \mathcal{U}_{1} \longrightarrow \mathbf{R}$ in Lagrangian neighbourhood coordinates by:

$$
H_{1}(q, p)=\tau(q, p) H(q)
$$

and to extend $H_{1}$ outside $\mathcal{U}_{1}$, simply make it zero.

The previous extension must now be modified near the boundary. First, choose a tubular neighbourhood $\mathcal{U}_{2}$ of $\partial \bar{M}_{\alpha}$ in which symplectic coordinates can be chosen as in Lemma 22. Suppose that the number $w_{2}$ characterizes the width of $\mathcal{U}_{2}$ in the sense that if $\left(x, y ; s^{1}, s^{2}\right)$ denotes a point in $T^{*} \partial \bar{M}_{\alpha} \times \mathbf{R}^{2}$, then it belongs to $\mathcal{U}_{2}$ if $\max \left\{\|y\|,\left|s^{1}\right|,\left|s^{2}\right|\right\}<w_{2}$. Now let $\eta_{0}: \mathbf{R} \longrightarrow \mathbf{R}$ be a smooth, positive cut-off function that is bounded by 1 , vanishes outside the interval $[0,1]$, and is equal to 1 inside the interval $[0,1 / 2]$. Define the extension $H_{2}: \mathcal{U}_{2} \longrightarrow \mathbf{R}$ in the $\left(x, y ; s^{1}, s^{2}\right)$ coordinates by

$$
H_{2}\left(x, y ; s^{1}, s^{2}\right)=\eta_{0}\left(\frac{\|y\|}{w_{2}}\right) \eta_{0}\left(\frac{\left|s^{2}\right|}{w_{2}}\right) \eta_{0}\left(\frac{\left|s^{1}\right|}{w_{2}}\right) H\left(x, s^{1}\right)
$$

and once again, let it to be zero outside $\mathcal{U}_{2}$.

The complete extension of the function $H$ that is desired will come from smoothly interpolating between the extensions $H_{1}$ and $H_{2}$. Denote by $\eta_{1}$ the function of $\mathbf{R}^{2 n}$ given by extending the function $\eta_{0}\left(\left|s^{1}\right| / w_{2}\right)$ defined in $\mathcal{U}_{2}$ to all of $\mathbf{R}^{2 n}$ by setting it equal to zero in $\mathbf{R}^{2 n} \backslash \mathcal{U}_{2}$.

Definition 23. For any $H \in C^{2, \beta}\left(\bar{M}_{\alpha}\right)$, the extension of $H$ to $\mathbf{R}^{2 n}$ is denoted by $H_{e}$ and is defined by the equation

$$
H_{e}(x)=\left(1-\eta_{1}(x)\right) H_{1}(x)+H_{2}(x)
$$

for any point $x$ in $\mathbf{R}^{2 n}$.

The following proposition shows that, with the proper boundary conditions on the functions $H$, the deformations $\phi_{H_{e}}^{1}$ deform $\bar{M}_{\alpha}$ in the desired manner.

Proposition 24. Let $H \in C^{2, \beta}\left(\bar{M}_{\alpha}\right)$ and suppose that $H$ satisfies $\left.Z(H)\right|_{\partial \bar{M}_{\alpha}}=0$. Then the family of submanifolds $\phi_{H_{e}}^{t}\left(\bar{M}_{\alpha}\right)$ is a Lagrangian 
deformation of $\bar{M}_{\alpha}$ and the family of boundaries $\phi_{H_{e}}^{t}\left(\partial \bar{M}_{\alpha}\right)$ remains on the scaffold $W$. Furthermore, the deformation vector field associated to this family of submanifolds is the vector field $X_{H}$ which satisfies $\left.X_{H}\right\rfloor \omega=\mathrm{d} H$ on $\bar{M}_{\alpha}$.

Proof. The proof of this proposition is a straightforward algebraic calculation and can be found in [3].

DEFINITION OF $v_{e}$

First define the function $v: \partial \bar{M}_{\alpha} \rightarrow \mathbf{R}$. Recall that $\partial \bar{M}_{\alpha}$ consists of the disjoint union of the two separate boundary components $\partial M_{1}$ and $\partial M_{2}$.

Definition 25. Define $v: \partial \bar{M}_{\alpha} \longrightarrow \mathbf{R}$ by the prescription

$$
v(x)= \begin{cases}\frac{1}{\operatorname{Vol}\left(\partial M_{1}\right)} & x \in \partial M_{1} \\ \frac{-1}{\operatorname{Vol}\left(\partial M_{2}\right)} & x \in \partial M_{2}\end{cases}
$$

Note that this definition implies that $\int_{\partial \bar{M}_{\alpha}} v=0$; this fact will be used later.

Use the symplectic coordinates for the neighbourhood $\mathcal{U}_{2}$ guaranteed by Lemma 22 to extend $v$. Define the extended function $v_{e}: \mathcal{U}_{2} \longrightarrow \mathbf{R}$ by

$$
v_{e}\left(x, y ; s^{1}, s^{2}\right)=v(x) s^{1} \eta_{0}\left(\frac{\|y\|}{w_{2}}\right) \eta_{0}\left(\frac{\left|s^{1}\right|}{w_{2}}\right) \eta_{0}\left(\frac{\left|s^{2}\right|}{w_{2}}\right) .
$$

Extend $v_{e}$ outside $\mathcal{U}_{2}$ by setting it equal to zero there. Note that the function $v_{e}$ satisfies the property $Z\left(v_{e}\right)=\frac{\partial}{\partial s^{1}} v_{e}=v$ at the boundary.

The deformation vector field of the Hamiltonian deformation $\phi_{v_{e}}^{b}$ is the vector field that satisfies $\left.X_{v_{e}}\right\rfloor \omega=\mathrm{d} v_{e}$ on $\bar{M}_{\alpha}$. This vector field can be obtained from similar calculations to those carried out above in the definition of $H_{e}$, resulting in the expression

$$
\left.X_{v_{e}}\right|_{\partial \bar{M}_{\alpha}}=-v(x) \frac{\partial}{\partial s^{2}} .
$$

This is perpendicular to $W$. Consequently, the deformation $\phi_{v_{e}}^{b}$ moves $\partial \bar{M}_{\alpha}$ away from $W$.

The differential operator $F_{\alpha}$ describing minimal Lagrangian submanifolds near $\bar{M}_{\alpha}$ comes from combining the general consideration of Section 2.2 with the specific parametrization of nearby Lagrangian embeddings constructed in the previous paragraphs. 
Definition 26. Let $\mathcal{B}_{1, \alpha}$ be the Banach space of functions given in equation (34). Define the map $F_{\alpha}: \mathcal{B}_{1, \alpha} \times \mathbf{R}^{2} \rightarrow C^{0, \beta}\left(\bar{M}_{\alpha}\right)$ by

$$
F_{\alpha}(H, \theta, b)=\left\langle\left(\phi_{v_{e}}^{b} \circ \phi_{H_{e}}^{1} \circ \bar{h}_{\alpha}\right)^{*} \operatorname{Im}\left(\mathrm{e}^{\mathrm{i} \theta} \mathrm{d} z\right), \operatorname{Vol}_{\bar{M}_{\alpha}}\right\rangle_{\bar{M}_{\alpha}} .
$$

The linearization of this operator at the point $(0,0,0)$ is now easily calculated by applying the general result of Proposition 3, and is given by the following proposition.

Proposition 27. Let $(u, a, b) \in \mathcal{B}_{1, \alpha} \times \mathbf{R}^{2}$. Then the linearization of $F_{\alpha}$ at $(0,0,0)$ acting on the point $(u, a, b)$ is given by the formula

$$
\begin{aligned}
\mathrm{D} F_{\alpha}(0,0,0)(u, a, b)=- & \cos \left(\theta_{\bar{M}_{\alpha}}\right) \Delta_{\bar{M}_{\alpha}} u-\sin \left(\theta_{\bar{M}_{\alpha}}\right)\left\langle\vec{H}_{\bar{M}_{\alpha}}, \nabla u\right\rangle \\
& +a \cos \left(\theta_{\bar{M}_{\alpha}}\right)-b \Delta_{\bar{M}_{\alpha}} v_{e} .
\end{aligned}
$$

\subsection{The Weighted Schauder Norm.}

Lagrangian submanifolds close to $\bar{M}_{\alpha}$ are parametrized over the Banach space $\mathcal{B}_{1, \alpha} \times \mathbf{R}$ according to Definition 21. It remains to choose the norms for both $\mathcal{B}_{1, \alpha}$ and $C^{0, \beta}\left(\bar{M}_{\alpha}\right)$ in which the measurements of $C_{L}(\alpha), C_{N}(\alpha)$ and $F_{\alpha}(0,0,0)$ will be made. The usual $C^{k, \beta}$ norms will turn out to be unsuitable for exhibiting the explicit dependence on $\alpha$ of these quantities. The optimal norms for solving the deformation problem are weighted Schauder norms, whose weight function compensates for the two different scales of $\bar{M}_{\alpha}$, one within the neck region and one outside it.

The two different scales of $\bar{M}_{\alpha}$ are encoded in the radius of uniformity of the induced metric $g_{\bar{M}_{\alpha}}$ and is defined as follows. For each point $p$ of $\bar{M}_{\alpha}$, there is a radius $r(p)$ so that geodesic normal coordinates can be used in the ball $B_{r(p)}(p)$ and the metric coefficients are uniformly $C^{1, \beta}$-bounded in these coordinates. That is,

$$
\left|g_{i j}-\delta_{i j}\right|_{1, \beta, B_{r(p)}(p)}^{*} \leq 1,
$$

where $|\cdot|_{1, \beta}^{*}$ is the scale-invariant Schauder norm of $\mathbf{R}^{n}$ given by

$$
|u|_{k, \beta, B_{R}}^{*}=|u|_{0, B_{R}}+R|\nabla u|_{0, B_{R}}+\cdots+R^{k}\left|\nabla^{k} u\right|_{0, B_{R}}+R^{k+\beta}\left[\nabla^{k} u\right]_{\beta, B_{R}} .
$$

Here, $|\cdot|_{0, B_{R}}$ denotes the supremum norm over $B_{R}$ and $[\cdot]_{\beta, B_{R}}$ denotes the Hölder coefficient in $B_{R}$.

If $p$ is in the interior region of $\bar{M}_{\alpha}$ then $r(p) \geq \varepsilon r_{1}\left(\frac{p}{\varepsilon}\right)$ where $r_{1}$ gives the radius of uniformity of the metric in the unscaled Lawlor neck $N_{1}$. The 
behaviour of $r_{1}$ in $N_{1}$ is as follows. In $N_{1} \cap B_{a}(0)$ for some fixed radius $a$, $r_{1}(p)$ is bounded below by some fixed number $R_{\text {int }}$. In $N_{1} \cap\left(B_{a}(0)\right)^{c}$, the bound $r_{1}(p) \geq m_{1}\|p\|$, for some fixed rate $m_{1}$ because if $N_{1} \cap\left(B_{a}(0)\right)^{c}$ is close to a cone if $a$ is sufficiently large. Translating this behaviour back to $\bar{M}_{\alpha}$ is a matter of rescaling: $r(p) \geq \varepsilon R_{\text {int }}$ in $\varepsilon N_{1} \cap B_{\varepsilon a}(0)$ and $r(p) \geq m\|p\|$ in $\varepsilon N_{1} \cap\left(B_{\varepsilon a}(0)\right)^{c}$. Next, the value of $r(p)$ for $p$ in the exterior region $M_{1}^{\prime} \cup M_{2}^{\prime}$ is independent of $\alpha$ and is bounded below by some value $R_{\text {ext }}$. Thus $r(p)$ grows from size $\varepsilon R_{\text {int }}$ with constant rate until it reaches the value $R_{\text {ext }}$, which it attains in some ball of radius independent of $\alpha$.

The behaviour of the radius of uniformity of the induced metric of $\bar{M}_{\alpha}$ suggests that the desired weight function should be one which interpolates between the value $\varepsilon R_{\text {int }}$ in a ball of radius $\varepsilon a$ contained in the interior region $N^{\prime}$ and the value $R_{e x t}$ in some part of the exterior region $M_{1}^{\prime} \cup M_{2}^{\prime}$. Let $R=\min \left\{R_{\text {int }}, R_{\text {ext }}\right\}$ and choose any $\beta \in(0,1)$. It is easy to verify that a smooth and increasing function of the following form exists on $\bar{M}_{\alpha}$.

Definition 28. Let $\rho: \bar{M}_{\alpha} \longrightarrow \mathbf{R}$ be a function of the form:

$$
\rho(x)= \begin{cases}R \varepsilon & x \in \bar{M}_{\alpha} \cap B_{\varepsilon a}(0) \\ \text { Interpolation } & \left.x \in \bar{M}_{\alpha} \cap A n n_{\left(\varepsilon a, \varepsilon^{\beta} b\right.}(0)\right) \\ R & x \in \bar{M}_{\alpha} \cap\left(B_{\varepsilon^{\beta} b}(0)\right)^{c}\end{cases}
$$

where $b \in \mathbf{R}$ and the interpolation can be chosen so that $\rho$ is smooth and so that the following additional properties hold.

Property 1: The gradient of $\rho$ satisfies the bound $\|\nabla \rho\| \leq K \varepsilon^{-\beta}$ for some $K$ independent of $\alpha$ because $\rho$ grows from size $\varepsilon R$ to size $R$ in an annular region of width on the order of $\varepsilon^{\beta}$.

Property 2: Since $\rho=\varepsilon R$ in $B_{\varepsilon a}(0)$ and then grows to size 1 in the annulus $A n n_{\left(\varepsilon a, \varepsilon^{\beta} b\right)}(0)$, there is a constant $C$ independent of $\alpha$ so that $\rho(x) \geq C\|x\|$ in this annulus.

Property 3: Suppose $k \geq 1$. Then $\left[\rho^{k}\right]_{\beta, \bar{M}_{\alpha}} \leq C \varepsilon^{-\beta}$, where $C$ is independent of $\alpha$.

Proof. Choose two points $x$ and $x^{\prime}$ in $\bar{M}_{\alpha}$ and let $\gamma$ be the length-minimizing 
geodesic connecting these two points. Now calculate,

$$
\begin{aligned}
\frac{\left|\rho^{k}(x)-\rho^{k}\left(x^{\prime}\right)\right|}{\left(\operatorname{dist}\left(x, x^{\prime}\right)\right)^{\beta}} & =\frac{\left|\int_{\gamma}\left\langle\nabla\left(\rho^{k}\right), \dot{\gamma}\right\rangle \mathrm{d} s\right|}{\left(\operatorname{dist}\left(x, x^{\prime}\right)\right)^{\beta}} \\
& \leq\left\|\nabla \rho^{k}\right\|_{0, \bar{M}_{\alpha}}\left(\operatorname{dist}\left(x, x^{\prime}\right)\right)^{1-\beta} \\
& \leq k\left|\rho^{k-1}\right|_{0, \bar{M}_{\alpha}}\|\nabla \rho\|_{0, \bar{M}_{\alpha}}\left(\operatorname{diam}\left(\bar{M}_{\alpha}\right)\right)^{1-\beta} .
\end{aligned}
$$

The distance function used here is the distance function on $\bar{M}_{\alpha}$ corresponding to the induced metric. All quantities except the gradient term in the estimate (42) are bounded above by constants independent of $\alpha$, whereas the gradient is bounded by $K \varepsilon^{-\beta}$.

Property 4: Suppose $\beta \in(0,1)$. Then $\left[\rho^{\beta}\right]_{\beta, \bar{M}_{\alpha}} \leq C \varepsilon^{-\beta}$, where $C$ is independent of $\alpha$.

Proof. Choose two points $x$ and $x^{\prime}$ in $\bar{M}_{\alpha}$ which satisfy $\operatorname{dist}\left(x, x^{\prime}\right) \geq \varepsilon$. Then,

$$
\begin{aligned}
\frac{\left|\rho^{\beta}(x)-\rho^{\beta}\left(x^{\prime}\right)\right|}{\left(\operatorname{dist}\left(x, x^{\prime}\right)\right)^{\beta}} & \leq 2\left|\rho^{\beta}\right|_{0, \bar{M}_{\alpha}} \varepsilon^{-\beta} \\
& \leq 2 R^{\beta} \varepsilon^{-\beta} .
\end{aligned}
$$

Next, suppose $\operatorname{dist}\left(x, x^{\prime}\right)<\varepsilon$. Then,

$$
\begin{aligned}
\frac{\left|\rho^{\beta}(x)-\rho^{\beta}\left(x^{\prime}\right)\right|}{\left(\operatorname{dist}\left(x, x^{\prime}\right)\right)^{\beta}} & \leq\left\|\nabla \rho^{\beta}\right\|_{0, \bar{M}_{\alpha}} \varepsilon^{1-\beta} \\
& \leq \beta\left|\rho^{\beta-1}\right|_{0, \bar{M}_{\alpha}}\|\nabla \rho\|_{0, \bar{M}_{\alpha}} \varepsilon^{1-\beta} \\
& \leq \beta(R)^{\beta-1} K \varepsilon^{-\beta}
\end{aligned}
$$

since $\rho$ is bounded below by $R \varepsilon$. Taking the supremum over $\bar{M}_{\alpha}$ yields the desired result.

Property 5: Suppose $p<n$. Then $\left\|\rho^{-1}\right\|_{L^{p}\left(\bar{M}_{\alpha}\right)} \leq C$, where $C$ is a constant independent of $\alpha$. 
Proof. Choose $p<n$ and calculate as follows.

$$
\begin{aligned}
\int_{\bar{M}_{\alpha}} \rho^{-p} & =\int_{\bar{M}_{\alpha} \cap B_{\varepsilon a}(0)} \rho^{-p}+\int_{\bar{M}_{\alpha} \cap A n n_{\left(\varepsilon a, \varepsilon^{\beta} b\right)}(0)} \rho^{-p}+\int_{\bar{M}_{\alpha} \cap\left(B_{\varepsilon^{\beta} b}(0)\right)^{c}} \rho^{-p} \\
& \leq(R \varepsilon)^{-p} \operatorname{Vol}\left(B_{\varepsilon a}(0)\right)+C \int_{\varepsilon a}^{\varepsilon^{\beta} b} s^{n-p-1} \mathrm{~d} s+\int_{\left(B_{\varepsilon^{\beta} \beta_{b}}(0)\right)^{c^{-p}}} R^{-p} \text { (by Property 2) } \\
& \leq C\left(1+\varepsilon^{n-p}\right)
\end{aligned}
$$

which is bounded when $p<n$.

Property 6: There exists a constant $C$ independent of $\alpha$ so that $\rho(x) \varepsilon^{\beta} \leq$ $\operatorname{Cr}(x)$ for every $x \in \bar{M}_{\alpha}$, where $r(x)$ is the radius of uniformity of the metric coefficients at the point $x$.

Proof. The result is true by definition of $\rho$ and $r$ in the ball $B_{\varepsilon a}(0)$ as well as well as outside some large ball of radius independent of $\alpha$ where $r(p)$ is bounded below. Between these two regions where $r$ grows linearly, the gradient bound on $\rho$ implies that $\rho(x) \leq \rho(0)+K \varepsilon^{-\beta}\|x\|$ and thus

$$
\begin{aligned}
\rho(x) \varepsilon^{\beta} & \leq \varepsilon^{1+\beta}+K\|x\| \\
& \leq \frac{1}{m}\left(\frac{\varepsilon^{\beta}}{a}+K\right) r(x)
\end{aligned}
$$

using the fact that $r(x) \geq m\|x\|$ in the region in question. This leads to the desired estimate.

The $\rho$-weighted Schauder norms on $\bar{M}_{\alpha}$ that will be used to carry out the estimates of the Main Theorem are defined as follows.

Definition 29. Let $u$ be any $C^{k, \beta}$ function on $\bar{M}_{\alpha}$. The weighted $(k, \beta)$ Schauder norm of $u$ will be denoted $|u|_{C_{\rho}^{k, \beta}\left(\bar{M}_{\alpha}\right)}$ and is defined as

$|u|_{C_{\rho}^{k, \beta}\left(\bar{M}_{\alpha}\right)}=|u|_{0, \bar{M}_{\alpha}}+|\rho \nabla u|_{0, \bar{M}_{\alpha}}+\cdots+\left|\rho^{k} \nabla^{k} u\right|_{0, \bar{M}_{\alpha}}+\left[\rho^{k+\beta} \nabla^{k} u\right]_{\beta, \bar{M}_{\alpha}}$.

Here, $|\cdot|_{0, \bar{M}_{\alpha}}$ denotes the supremum norm and $[\cdot]_{\beta, \bar{M}_{\alpha}}$ denotes the Hölder coefficient on $\bar{M}_{\alpha}$; the norms and derivatives in this expression are those corresponding to the induced metric on $\bar{M}_{\alpha}$.

The differential operator $F_{\alpha}$ maps between the Banach spaces $\mathcal{B}_{1, \alpha} \times \mathbf{R}^{2}$ and $C^{0, \beta}\left(\bar{M}_{\alpha}\right)$. The analysis that will be performed in order to solve the equation $F_{\alpha}(u, a, b)=0$ requires a choice of norm to be made in both of these spaces. 
- Use the weighted norm $|u|_{C_{\rho}^{2, \beta}\left(\bar{M}_{\alpha}\right)}$ for functions $u$ in the Banach space $\mathcal{B}_{1, \alpha}$.

- Use the product norm $\|(u, a, b)\|_{C_{\rho}^{2, \beta}\left(\bar{M}_{\alpha}\right) \times \mathbf{R}^{2}}=\left(|u|_{C_{\rho}^{2, \beta}\left(\bar{M}_{\alpha}\right)}^{2}+a^{2}+b^{2}\right)^{1 / 2}$ for elements in the Banach space $\mathcal{B}_{1, \alpha} \times \mathbf{R}^{2}$.

- Use the weighted norm $\left|\rho^{2} f\right|_{C_{\rho}^{0, \beta}\left(\bar{M}_{\alpha}\right)}$ for functions $f$ in the Banach space $C^{0, \beta}\left(\bar{M}_{\alpha}\right)$.

It is easy to verify that $\mathcal{B}$ and $C^{0, \beta}\left(\bar{M}_{\alpha}\right)$ are indeed Banach spaces with these norms. Furthermore, $\mathrm{DF}_{\alpha}(0,0,0)$ is a bounded operator in the weighted norms.

Proposition 30. The linearization $\mathrm{D} F_{\alpha}(0,0,0)$ of $F_{\alpha}$ at the origin is a bounded operator between the space $\mathcal{B}_{1, \alpha} \times \mathbf{R}^{2}$ with norm $\|\cdot\|_{C_{\rho}^{2, \beta}\left(\bar{M}_{\alpha}\right) \times R^{2}}$ and the space $C^{0, \beta}\left(\bar{M}_{\alpha}\right)$ with norm $\left|\rho^{2} \cdot\right|_{C_{\rho}^{0, \beta}\left(\bar{M}_{\alpha}\right)}$.

Proof. Let $(u, a, b) \in \mathcal{B}_{1, \alpha} \times \mathbf{R}^{2}$. Then using the form of $\mathrm{DF}_{\alpha}(0,0,0)$ derived in Proposition 27,

$$
\begin{aligned}
\mid \rho^{2} \mathrm{D} F_{\alpha}(0,0,0) & \left.(u, a, b)\right|_{C_{\rho}^{0, \beta}\left(\bar{M}_{\alpha}\right)} \leq\left|\rho^{2} \cos \left(\theta_{\bar{M}_{\alpha}}\right) \Delta_{\bar{M}_{\alpha}} u\right|_{C_{\rho}^{0, \beta}\left(\bar{M}_{\alpha}\right)} \\
& +\left|\rho^{2} \sin \left(\theta_{\bar{M}_{\alpha}}\right)\left\langle\vec{H}_{\bar{M}_{\alpha}}, \nabla u\right\rangle_{\bar{M}_{\alpha}}\right|_{C_{\rho}^{0, \beta}\left(\bar{M}_{\alpha}\right)} \\
& +|a|\left|\rho^{2} \cos \left(\theta_{\bar{M}_{\alpha}}\right)\right|_{C_{\rho}^{0, \beta}\left(\bar{M}_{\alpha}\right)}-|b|\left|\rho^{2} \Delta_{\bar{M}_{\alpha}} v_{e}\right|_{C_{\rho}^{0, \beta}\left(\bar{M}_{\alpha}\right)}
\end{aligned}
$$

Each of the four terms in the expression above will be estimated in turn. Begin with the first term:

$$
\begin{aligned}
& \quad\left|\rho^{2} \cos \left(\theta_{\bar{M}_{\alpha}}\right) \Delta_{\bar{M}_{\alpha}} u\right|_{C_{\rho}^{0, \beta}\left(\bar{M}_{\alpha}\right)} \\
& \leq\left|\rho^{2} \cos \left(\theta_{\bar{M}_{\alpha}}\right) \Delta_{\bar{M}_{\alpha}} u\right|_{0, \bar{M}_{\alpha}}+\left|\cos \left(\theta_{\bar{M}_{\alpha}}\right)\right|_{0, \bar{M}_{\alpha}} \cdot\left[\rho^{2+\beta} \Delta_{\bar{M}_{\alpha}} u\right]_{\beta, \bar{M}_{\alpha}} \\
& \quad \quad+\left[\cos \left(\theta_{\bar{M}_{\alpha}}\right)\right]_{\beta, \bar{M}_{\alpha}} \cdot\left|\rho^{\beta}\right|_{0, \bar{M}_{\alpha}} \cdot\left|\rho^{2} \Delta_{\bar{M}_{\alpha}} u\right|_{0, \bar{M}_{\alpha}} \\
& \leq\left(1+\left[\cos \left(\theta_{\bar{M}_{\alpha}}\right)\right]_{\beta, \bar{M}_{\alpha}} \cdot\left|\rho^{\beta}\right|_{0, \bar{M}_{\alpha}}\right)|u|_{C_{\rho}^{2, \beta}\left(\bar{M}_{\alpha}\right)}
\end{aligned}
$$

by definition of the $C_{\rho}^{2, \beta}$ norm and the bounds (29) on the trigonometric functions of $\theta_{\bar{M}_{\alpha}}$. Now,

$$
\left|\rho^{2} \cos \left(\theta_{\bar{M}_{\alpha}}\right) \Delta_{\bar{M}_{\alpha}} u\right|_{C_{\rho}^{0, \beta}\left(\bar{M}_{\alpha}\right)} \leq C\left(1+\alpha^{1-\beta}\right)|u|_{C_{\rho}^{2, \beta}\left(\bar{M}_{\alpha}\right)},
$$


by the bounds on the function $\rho$ and on the Hölder norm of the cosine term.

The second term in equation (43) can be estimated in a manner similar to that used for the first term, this time using the bounds on the mean curvature from Proposition 15. One obtains

$$
\left|\rho^{2} \sin \left(\theta_{\bar{M}_{\alpha}}\right)\left\langle\vec{H}_{\bar{M}_{\alpha}}, \nabla u\right\rangle_{\bar{M}_{\alpha}}\right|_{C_{\rho}^{0, \beta}\left(\bar{M}_{\alpha}\right)} \leq C \alpha
$$

Finally, deal with the remaining two terms in (43). It is trivial to show that the third term is bounded above by a constant independent of $\alpha$. For the last term, recall that $v_{e}$ is explicitly independent of $\alpha$ and is nonzero only near the boundary of $\bar{M}_{\alpha}$ where $\rho$ is independent of $\alpha$; thus bounding this term above by a constant independent of $\alpha$ is trivial as well.

The considerations of the previous two paragraphs lead to the estimates

$$
\left|\rho^{2} \mathrm{D} F_{\alpha}(0,0,0)(u, a, b)\right|_{C_{\rho}^{0, k}\left(\bar{M}_{\alpha}\right)} \leq C\left(|u|_{C_{\rho}^{2, \beta}\left(\bar{M}_{\alpha}\right)}+|a|+|b|\right) .
$$

Since the norm on the right hand side above is equivalent to the $\|$. $\|_{C_{\rho}^{2, \beta}\left(\bar{M}_{\alpha}\right) \times \mathbf{R}^{2}}$ norm, the proof of the proposition is complete.

The next theorem shows that an elliptic estimate for the operator $\Delta_{\bar{M}_{\alpha}}$ can be found using the weighted Schauder norms and that the constant appearing there is independent (or nearly so) of $\alpha$.

Theorem 31 (Elliptic Estimate). There is a constant $C_{E l l}$ independent of $\alpha$ so that the elliptic estimate

$$
|u|_{C_{\rho}^{2, \beta}\left(\bar{M}_{\alpha}\right)} \leq C_{E l l}\left(\varepsilon^{-2 \beta}\left|\rho^{2} \Delta_{\bar{M}_{\alpha}} u\right|_{C_{\rho}^{0, \beta}\left(\bar{M}_{\alpha}\right)}+|u|_{0, \bar{M}_{\alpha}}\right)
$$

holds for any $C^{2, \beta}$ function $u$ on $\bar{M}_{\alpha}$ with $\alpha \leq A_{2}$ and satisfying the Neumann boundary condition $Z(u)=0$ on $\partial \bar{M}_{\alpha}$.

Proof. The strategy for proving the elliptic estimate (45) is to piece together local elliptic estimates, valid in coordinate charts in which the metric coefficients are uniformly bounded. These local elliptic estimates can be phrased as follows and their proof can be found in any standard textbook on elliptic theory, for instance [4].

FACT: Suppose $P: C^{2, \beta}(\Omega) \longrightarrow C^{0, \beta}(\Omega)$ is a second order elliptic operator defined on a domain $\Omega$ contained in $\mathbf{R}^{n}$. Then the following estimates are valid for any $C^{2, \beta}$ function $u$ on $\Omega$. Here, $\theta \in(0,1)$ is arbitrary and $C_{l o c}$ is 
a constant which depends only on $\theta, \beta$, the $C^{0, \beta}$ norm of the coefficients of $P$ and the dimension $n$. The norms used here are the local, scale invariant Schauder norms introduced in (41).

1. Let $x$ belong to the interior of $\Omega$. Then,

$$
|u|_{2, \beta, B_{\theta R}(x)}^{*} \leq C_{l o c}\left(R^{2}|P u|_{0, \beta, B_{R}(x)}^{*}+|u|_{0, B_{R}(x)}\right)
$$

where $B_{R}(x)$ is a ball centered at $x$ and contained in the interior of $\Omega$.

2. Let $x$ belong to $\partial \Omega$ and define $B_{R}^{+}(x)=B_{R}(x) \cap \Omega$ and $U_{R}(x)=$ $B_{R}(x) \cap \partial \Omega$. Then,

$$
|u|_{2, \beta, B_{\theta R}^{+}(x)}^{*} \leq C_{l o c}\left(R^{2}|P u|_{0, \beta, B_{R}^{+}(x)}^{*}+|u|_{0, B_{R}^{+}(x)}+R|Z(u)|_{1, \beta, U_{R}(x)}^{*}\right)
$$

where $Z$ is the conormal vector field of the boundary of $\Omega$.

The proof of the global elliptic estimate for $\Delta_{\bar{M}_{\underline{\alpha}}}$ on all of $\bar{M}_{\alpha}$ will establish in two separate calculations that for any $x \in \bar{M}_{\alpha}$,

$$
|u(x)|+\rho(x)\|\nabla u(x)\|+\rho^{2}(x)\left\|\nabla^{2} u(x)\right\| \leq Q
$$

and

$$
\frac{\left\|\rho^{2+\beta}(x) \nabla^{2} u(x)-\rho^{2+\beta}\left(x^{\prime}\right) \nabla^{2} u\left(x^{\prime}\right)\right\|}{\left(\operatorname{dist}\left(x, x^{\prime}\right)\right)^{\beta}} \leq Q,
$$

where $Q$ refers to the right hand side of the inequality (45) above. If this is established for all $x \in \bar{M}_{\alpha}$ (or $x$ and $x^{\prime}$ in $\bar{M}_{\alpha}$ in the second case), then the theorem follows by taking the supremum.

Begin by choosing a point $x$ in the interior of $\bar{M}_{\alpha}$. For the first calculation, let $s=\rho(x)$ and recall that $\|\nabla \rho(y)\| \leq K \varepsilon^{-\beta} \equiv K_{\varepsilon}$ for all $y \in \bar{M}_{\alpha}$. Property 6 of the weight function shows that one can assume, without loss of generality, that the constant $K_{\varepsilon}$ is such that $\frac{s}{2 K_{\varepsilon}}$ is less than $r(x)$, which is the radius of uniformity of the metric coefficients at $x$. Consequently, local coordinates in which the coefficients of the metric are uniformly bounded can be used within $B_{s / 2 K_{\varepsilon}}(x)$. Furthermore, if $y$ is any point in $B_{s / 2 K_{\varepsilon}}(x)$, then $|\rho(y)-\rho(x)| \leq K_{\varepsilon}|x-y|$ and this implies

$$
|\rho(y)| \geq|\rho(x)|-K_{\varepsilon}|x-y| \geq \frac{s}{2} .
$$


By a similar argument, $|\rho(y)| \leq \frac{3 s}{2}$ in $B_{s / 2 K_{\varepsilon}}(x)$. Now choose $\theta \in(0,1)$ and argue as follows. First, by simple algebra,

$$
\begin{aligned}
|u(x)|+s\|\nabla u(x)\| & +s^{2}\left\|\nabla^{2} u(x)\right\| \leq|u(x)| \\
& +C \varepsilon^{-2 \beta}\left(\frac{\theta s}{2 K_{\varepsilon}}\|\nabla u(x)\|+\left(\frac{\theta s}{2 K_{\varepsilon}}\right)^{2}\left\|\nabla^{2} u(x)\right\|\right)
\end{aligned}
$$

where $C$ depends only on $\theta$ and $K$. But now, the definition of the local $|\cdot|^{*}$ Schauder norm and the local elliptic estimate in the ball $B_{s / 2 K_{\varepsilon}}(x)$ can be applied to give

$$
\begin{aligned}
& |u(x)|+s\|\nabla u(x)\|+s^{2}\left\|\nabla^{2} u(x)\right\| \\
& \leq C \varepsilon^{-2 \beta}\left(s^{2}\left|\Delta_{\bar{M}_{\alpha}} u\right|_{0, B_{s / 2 K_{\varepsilon}}(x)}+s^{2+\beta}\left[\Delta_{\bar{M}_{\alpha}} u\right]_{\beta, B_{s / 2 K_{\varepsilon}}(x)}\right)+|u|_{0, \bar{M}_{\alpha}},
\end{aligned}
$$

where $C$ now depends on $C_{l o c}$. The first term in equation (50) is easy to handle:

$$
\left|\rho^{2} \Delta_{\bar{M}_{\alpha}} u\right|_{0, \bar{M}_{\alpha}} \geq \sup _{y \in B_{s / 2 K_{\varepsilon}}(x)}\left|\rho^{2}(y) \Delta_{\bar{M}_{\alpha}} u(y)\right| \geq \frac{s^{2}}{4}\left|\Delta_{\bar{M}_{\alpha}} u\right|_{0, B_{s / 2 K_{\varepsilon}}(x)}
$$

by the lower bounds on $\rho$. For the second term of (50), choose $y$ and $y^{\prime}$ in $B_{s / 2 K_{\varepsilon}}(x)$ and estimate

$$
\begin{aligned}
& \frac{\left|\rho^{2+\beta}(y) \Delta_{\bar{M}_{\alpha}} u(y)-\rho^{2+\beta}\left(y^{\prime}\right) \Delta_{\bar{M}_{\alpha}} u\left(y^{\prime}\right)\right|}{\left|y-y^{\prime}\right|^{\beta}} \\
& \geq\left|\rho^{2+\beta}(y)\right| \frac{\left|\Delta_{\bar{M}_{\alpha}} u(y)-\Delta_{\bar{M}_{\alpha}} u\left(y^{\prime}\right)\right|}{\left|y-y^{\prime}\right|^{\beta}} \\
& -(2+\beta)\left|\rho^{1+\beta}\right|_{0, B_{s / 2 K_{\varepsilon}}(x)}\|\nabla \rho\|_{0, B_{s / 2 K_{\varepsilon}}(x)}\left|y-y^{\prime}\right|^{1-\beta}\left|\Delta_{\bar{M}_{\alpha}} u\left(y^{\prime}\right)\right| \\
& \geq C\left(s^{2+\beta} \frac{\left|\Delta_{\bar{M}_{\alpha}} u(y)-\Delta_{\bar{M}_{\alpha}} u\left(y^{\prime}\right)\right|}{\left|y-y^{\prime}\right|^{\beta}}-K_{\varepsilon}^{\beta} \frac{s^{2}}{4}\left|\Delta_{\bar{M}_{\alpha}} u\right|_{0, B_{s / 2 K_{\varepsilon}}(x)}\right) \\
& \geq C\left(s^{2+\beta} \frac{\left|\Delta_{\bar{M}_{\alpha}} u(y)-\Delta_{\bar{M}_{\alpha}} u\left(y^{\prime}\right)\right|}{\left|y-y^{\prime}\right|^{\beta}}-\varepsilon^{-2 \beta}\left|\rho^{2} \Delta_{\bar{M}_{\alpha}} u\right|_{0, B_{s / 2 K_{\varepsilon}}(x)}\right)
\end{aligned}
$$

using the bounds on $\rho$ and $\|\nabla \rho\|$ as well as the result in equation (51). If the supremum of (52) over all $y$ and $y^{\prime}$ in the ball $B_{s / 2 K_{\varepsilon}}(x)$ is taken, then the inequality

$$
s^{2+\beta}\left[\Delta_{\bar{M}_{\alpha}} u\right]_{\beta, B_{s / 2 K_{\varepsilon}}(x)} \leq C \varepsilon^{-2 \beta}\left|\rho^{2} \Delta_{\bar{M}_{\alpha}} u\right|_{C_{\rho}^{0, \beta}\left(\bar{M}_{\alpha}\right)}
$$


follows, again by applying equation (51). Substituting the inequality (53) along with the previous inequality (51) into equation (50) yields the first estimate (48).

For the sake of brevity, the calculation of the second inequality in (49) will be omitted because it is essentially the same as the previous calculation. Nevertheless, all the details can be found in [3]. Finally, the case of $x \in$ $\partial \bar{M}_{\alpha}$ follows trivially from the local elliptic boundary estimate because the boundary of $\bar{M}_{\alpha}$ is independent of $\alpha$.

\section{Analysis of the Linearized Operator.}

\subsection{Outline.}

In order to invoke the Inverse Function Theorem to produce solutions of the equation $F_{\alpha}(H, \theta, b)=0$, the linearized operator $\mathrm{D} F_{\alpha}(0,0,0)$ must be a bijection satisfying the estimates required by the Inverse Function Theorem. The injectivity of the linearized operator will be established by producing a lower bound of the form $\left|\rho^{2} \mathrm{D} F_{\alpha}(0,0,0)(u, a, b)\right|_{C_{\rho}^{0, \beta}\left(\bar{M}_{\alpha}\right)} \geq$ $C_{L}(\alpha)\|(u, a, b)\|_{C_{\rho}^{2, \beta}\left(\bar{M}_{\alpha}\right) \times \mathbf{R}^{2}}$ for any $u \in \mathcal{B}_{1, \alpha}$ and $(a, b) \in \mathbf{R}^{2}$. Let

$$
\begin{gathered}
P_{\alpha}=\cos \left(\theta_{\bar{M}_{\alpha}}\right) \Delta_{\bar{M}_{\alpha}}+\sin \left(\theta_{\bar{M}_{\alpha}}\right) \vec{H}_{\bar{M}_{\alpha}} \cdot \nabla \\
\psi_{0, \alpha}=\cos \left(\theta_{\bar{M}_{\alpha}}\right) \\
\psi_{1, \alpha}=-\Delta_{\bar{M}_{\alpha}} v_{e} .
\end{gathered}
$$

The desired estimate is now equivalent to:

$$
\left|\rho^{2} P_{\alpha} u-a \psi_{0, \alpha}+b \psi_{1, \alpha}\right|_{C_{\rho}^{0, \beta}\left(\bar{M}_{\alpha}\right)} \geq C_{L}(\alpha)\left(|u|_{C_{\rho}^{2, \beta}\left(\bar{M}_{\alpha}\right)}^{2}+a^{2}+b^{2}\right)^{1 / 2} .
$$

Such an estimate will be developed in Sections 5.2 through 5.6 and the precise dependence of $C_{L}$ on $\alpha$ will of course be determined as well. It will be shown that the choice of parametrization made in the previous Section is enough to ensure that $C_{L}(\alpha)$ is nearly independent of $\alpha$. The surjectivity of the linearized operator will follow in a more or less straightforward manner from the results of the analysis leading up to the injectivity estimate and will be presented in Section 5.7.

The lower bound (54) comes from combining four separate results, and it is worthwhile to indicate in general terms how this will be done before proceeding with the details. The starting point is an estimate on the second Neumann eigenvalue of the Laplacian on $\bar{M}_{\alpha}$. This is the key estimate of 
this paper. It is purely global in nature and is of the form

$$
\left\|\Delta_{\bar{M}_{\alpha}} u\right\|_{L^{2}\left(\bar{M}_{\alpha}\right)} \geq C\|u\|_{L^{2}\left(\bar{M}_{\alpha}\right)}
$$

for functions $u$ perpendicular to 1 and to $S_{\alpha}$, the first eigenfunction of the Laplacian on $\bar{M}_{\alpha}$. Here, $C$ is independent of $\alpha$.

The second step is to deduce an estimate similar to the one above but in the weighted $C_{\rho}^{k, \beta}$ Schauder norms; in other words, one of the form

$$
\left|\rho^{2} \Delta_{\bar{M}_{\alpha}} u\right|_{C_{\rho}^{0, \beta}\left(\bar{M}_{\alpha}\right)} \geq C \varepsilon^{2 \beta}|u|_{C_{\rho}^{2, \beta}\left(\bar{M}_{\alpha}\right)} \cdot
$$

This estimate will be found by combining the elliptic estimate for $\Delta_{\bar{M}_{\alpha}}$ derived in Section 4.2 with the De Giorgi Nash estimate for $\Delta_{\bar{M}_{\alpha}} u$.

The third step is to deduce the desired injectivity estimate $P_{\alpha}$. This estimate, namely

$$
\left|\rho^{2}\left(\cos \left(\theta_{\bar{M}_{\alpha}}\right) \Delta_{\bar{M}_{\alpha}} u+\sin \left(\theta_{\bar{M}_{\alpha}}\right) \vec{H}_{\bar{M}_{\alpha}} \cdot \nabla u\right)\right|_{C_{\rho}^{0, \beta}\left(\bar{M}_{\alpha}\right)} \geq C \varepsilon^{2 \beta}|u|_{C_{\rho}^{2, \beta}\left(\bar{M}_{\alpha}\right)},
$$

holds because the mean curvature trigonometric terms can be controlled when $\alpha$ is small.

The final component of the injectivity estimate is to incorporate the $\psi_{0, \alpha}$ and $\psi_{1, \alpha}$ factors into the estimate found in the preceding step. This is a consequence of the fact that functions in $\mathcal{B}_{1, \alpha}$ are orthogonal to 1 and $S_{\alpha}$.

\subsection{Second Eigenvalue Estimate for $\Delta_{\bar{M}_{\alpha}}$.}

The fundamental fact that will guarantee the injectivity of the linearized operator $\mathrm{D} F_{\alpha}(0,0,0)$ is that the second eigenvalue $\nu_{2}$ of $\Delta_{\bar{M}_{\alpha}}$ is bounded below independently of $\alpha$. This bound will be derived by piecing together two $\alpha$-independent inequalities that already hold on $\bar{M}_{\alpha}$. The first of these is essentially the Poincaré inequality of the original special Lagrangian submanifolds $M_{1}$ and $M_{2}$ comprising $M$.

Proposition 32. Suppose $u$ belongs to $H^{1}\left(\bar{M}_{\alpha}\right)$, satisfies $\int_{\bar{M}_{\alpha}} u=0$, and has support contained in only one of the factors $M_{1}^{\prime}$ or $M_{2}^{\prime}$. Then the function $u$ satisfies the inequality

$$
\int_{\bar{M}_{\alpha}}\|\nabla u\|^{2} \geq E_{0} \int_{\bar{M}_{\alpha}} u^{2}
$$

where $E_{0}$ is a constant independent of $\alpha$, 
Proof. Let $E_{0}=\min \left\{\lambda_{1}, \lambda_{2}\right\}$, where each $\lambda_{i}$ is the first Neumann eigenvalue of $M_{i}$. The result of the proposition is now an elementary consequence of the Poincaré inequalities $\int_{M_{i}}\|\nabla u\|^{2} \geq \lambda_{i} \int_{M_{i}} u^{2}$ and the fact that $M_{i}^{\prime} \subset M_{i}$.

The second inequality that is valid on $\bar{M}_{\alpha}$ holds independently of $\alpha$ even in the neck region.

Proposition 33. There is a number $A_{3}$ with $0<A_{3} \leq A_{2}$ and a geometric constant $E_{M S}$ independent of $\alpha$ so that if $\alpha \leq A_{3}$ then the inequality

$$
\int_{\bar{M}_{\alpha}}|u|^{2} \leq E_{M S} \int_{\bar{M}_{\alpha}}\|\nabla u\|^{2}
$$

holds for any $H^{1}$ function $u$ that vanishes in a neighbourhood of $\partial \bar{M}_{\alpha}$.

Proof. The Michael-Simon inequality [21] for a submanifold $M$ with boundary in $\mathbf{R}^{2 n}$ states

$$
\left(\int_{M}|v|^{\frac{n}{n-1}}\right)^{\frac{n-1}{n}} \leq C \int_{M}\left(\|\nabla v\|+\left\|\vec{H}_{M}\right\| \cdot|v|\right)
$$

for any function $v \in H^{1}(M)$ which vanishes in some neighbourhood of the boundary $\partial M$. Here, $\vec{H}_{M}$ is the mean curvature vector of $M$ and $C$ is a constant depending only on $n$.

According to Proposition 15, the mean curvature vector of $\bar{M}_{\alpha}$ is pointwise bounded everywhere in the transition region $T_{1} \cup T_{2}$ and vanishes everywhere else. Thus integral norms of the magnitude of $\vec{H}_{\bar{M}_{\alpha}}$ can be made as small as desired by choosing the parameter $\alpha$ small enough. This makes it possible to absorb the mean curvature term in (59) into the left hand side, thereby producing the inequality (58). This calculation will be omitted because it is a straightforward application of the Hölder inequality upon substituting $v=u^{2(n-1) / n}$.

The desired estimate on $\nu_{2}$ now comes from combining the Poincaré inequalities on the separate pieces $M_{i}^{\prime}$ with the Michael-Simon result in the neck region $T_{1} \cup N^{\prime} \cup T_{2}$.

Theorem 34 (Second Eigenvalue Estimate). If $\alpha \leq A_{3}$, then the second Neumann eigenvalue $\nu_{2}$ of the Laplacian on $\bar{M}_{\alpha}$ is bounded below by a 
geometric constant $C_{2}$ independent of $\alpha$. Consequently, the inequality

$$
\left\|\Delta_{\bar{M}_{\alpha}} u\right\|_{L^{2}\left(\bar{M}_{\alpha}\right)} \geq C_{2}\|u\|_{L^{2}\left(\bar{M}_{\alpha}\right)}
$$

holds for all $u \in \mathcal{B}_{1, \alpha}$.

Proof. The lower bound $C_{2}$ will be deduced using the max-min characterization of the second eigenvalue, namely that

$$
\nu_{2} \geq \inf _{\substack{u \in H^{1}\left(\bar{M}_{\alpha}\right) \\ u \neq 0}}\left\{\frac{\int_{\bar{M}_{\alpha}}\|\nabla u\|^{2}}{\int_{\bar{M}_{\alpha}} u^{2}}: \int_{\bar{M}_{\alpha}} u \cdot \phi_{1}=\int_{\bar{M}_{\alpha}} u \cdot \phi_{2}=0\right\}
$$

for any specific choice of $\phi_{1}$ and $\phi_{2}$. Begin by making such a choice.

Recall that the submanifold $\bar{M}_{\alpha}$ consists of the union of the exterior components $M_{1}^{\prime}$ and $M_{2}^{\prime}$ of $M$, along with the neck region $T_{1} \cup N^{\prime} \cup T_{2}$. Denote this latter region simply by $N$. Define the functions $\phi_{i}: \bar{M}_{\alpha} \longrightarrow \mathbf{R}$ by the prescription

$$
\begin{aligned}
& \phi_{1}=\chi_{M_{1}^{\prime}}+\chi_{M_{2}^{\prime}} \\
& \phi_{2}=\chi_{M_{1}^{\prime}}-\chi_{M_{2}^{\prime}}
\end{aligned}
$$

where $\chi_{U}$ refers to the characteristic function of the subset $U$ of $\bar{M}_{\alpha}$.

Let $u$ be any $C^{1}$ function on $\bar{M}_{\alpha}$. Suppose that it has $L^{2}$ norm equal to one and satisfies the orthogonality conditions $\int_{\bar{M}_{\alpha}} \phi_{1} \cdot u=0$ and $\int_{\bar{M}_{\alpha}} \phi_{2} \cdot u=$ 0 . These two conditions give

$$
\int_{M_{1}^{\prime}} u=0 \quad \text { and } \quad \int_{M_{2}^{\prime}} u=0 .
$$

Choose any $\delta \in(0,1)$ and consider the two separate cases: either $\int_{M_{1}^{\prime} \cup M_{2}^{\prime}} u^{2} \geq 1-\delta$ and thus $\int_{N} u^{2} \leq \delta$; or else $\int_{M_{1}^{\prime} \cup M_{2}^{\prime}} u^{2} \leq 1-\delta$ and thus $\int_{N} u^{2} \geq \delta$. It will be possible to show that there is always a choice of $\delta \in(0,1)$ so that in both cases above, the inequality $\int_{\bar{M}_{\alpha}}\|\nabla u\|^{2} \geq C_{2}$ holds for some positive geometric constant $C_{2}$ independent of $\alpha$.

CASE 1: $\int_{M_{1}^{\prime} \cup M_{2}^{\prime}} u^{2} \geq 1-\delta$ and $\int_{N} u^{2} \leq \delta$.

Calculate as follows:

$$
\begin{aligned}
\int_{\bar{M}_{\alpha}}\|\nabla u\|^{2} & =\int_{M_{1}^{\prime}}\|\nabla u\|^{2}+\int_{N}\|\nabla u\|^{2}+\int_{M_{2}^{\prime}}\|\nabla u\|^{2} \\
& \geq \int_{M_{1}}\|\nabla \bar{u}\|^{2}+\int_{M_{2}}\|\nabla \bar{u}\|^{2}
\end{aligned}
$$


where $\bar{u}$ is the $L^{2}$ function on $\bar{M}_{\alpha}$ which is equal to $u$ on $M_{1}^{\prime} \cup M_{2}^{\prime}$ and equal to zero in $N$. The Poincaré inequality of Proposition (32) for functions supported away from the neck region can be applied (since $H^{1}\left(\bar{M}_{\alpha}\right)$ is dense in $\left.L^{2}\left(\bar{M}_{\alpha}\right)\right)$ to give

$$
\begin{aligned}
\int_{\bar{M}_{\alpha}}\|\nabla u\|^{2} & \geq E_{0} \int_{\bar{M}_{\alpha}} \bar{u}^{2} \\
& =E_{0} \int_{M_{1}^{\prime} \cup M_{2}^{\prime}} u^{2} \\
& \geq E_{0}(1-\delta) .
\end{aligned}
$$

CASE 2: $\int_{M_{1}^{\prime} \cup M_{2}^{\prime}} u^{2} \leq 1-\delta$ and $\int_{N} u^{2} \geq \delta$.

Choose a function $\eta: \bar{M}_{\alpha} \longrightarrow \mathbf{R}$ with the following properties. Let $\eta$ satisfy $0 \leq \eta \leq 1$, be equal to one in $N$ and vanish in a neighbourhood of the boundary of $\bar{M}_{\alpha}$. Moreover, choose $\eta$ such that $\|\nabla \eta\|$ is bounded above by a constant $K$ that is independent of $\alpha$. Write $u=\eta u+(1-\eta) u$. The function $\eta u$ is thus a member of $H^{1}\left(\bar{M}_{\alpha}\right)$ and vanishes in a neighbourhood of the boundary. Consequently, the Poincaré inequality of Proposition (33) is valid for $\eta u$, and this yields the inequalities

$$
\begin{aligned}
\int_{\bar{M}_{\alpha}}\|\nabla u\|^{2} & =\int_{\bar{M}_{\alpha}}\|\nabla(\eta u+(1-\eta) u)\|^{2} \\
& =\int_{\bar{M}_{\alpha}}\|\nabla \eta u\|^{2}+2 \int_{\bar{M}_{\alpha}}\langle\nabla \eta u, \nabla(1-\eta) u\rangle+\int_{\bar{M}_{\alpha}}\|\nabla(1-\eta) u\|^{2} \\
& \geq E_{M S} \int_{N} u^{2}+2 \int_{\bar{M}_{\alpha}}(1-2 \eta) u \nabla u \cdot \nabla \eta-2 K^{2} \int_{M_{1}^{\prime} \cup M_{2}^{\prime}} u^{2} \\
& \geq E_{M S} \delta+2 \int_{\bar{M}_{\alpha}}(1-2 \eta) u \nabla u \cdot \nabla \eta-2 K^{2}(1-\delta)
\end{aligned}
$$

by the Michael-Simon Poincaré inequality and the fact that $\nabla \eta$ is equal to zero in $N$. It remains only to deal with the cross term. By the CauchySchwarz inequality,

$$
\begin{aligned}
2 \int_{\bar{M}_{\alpha}}(1-2 \eta) u \nabla u \cdot \nabla \eta & \geq-2 \int_{\bar{M}_{\alpha}}|u||1-2 \eta||\nabla u \cdot \nabla \eta| \\
& \geq-2 \int_{\bar{M}_{\alpha}}|u|\|\nabla u\|\|\nabla \eta\| .
\end{aligned}
$$

The fact that $|1-2 \eta| \leq 1$ has been used here. Now apply the Hölder inequality and the Schwarz inequality $2 a b \leq a^{2}+b^{2}$ to the integral on the 
right hand side above to obtain:

$$
\begin{aligned}
2 \int_{\bar{M}_{\alpha}}(1-2 \eta) u \nabla u \cdot \nabla \eta & \geq-2\left(\int_{\bar{M}_{\alpha}}\|\nabla u\|^{2}\right)^{\frac{1}{2}}\left(\int_{\bar{M}_{\alpha}}|u|^{2}\|\nabla \eta\|^{2}\right)^{\frac{1}{2}} \\
& \geq-\int_{\bar{M}_{\alpha}}\|\nabla u\|^{2}-K^{2} \int_{M_{1}^{\prime} \cup M_{2}^{\prime}}|u|^{2} \\
& \geq-\int_{\bar{M}_{\alpha}}\|\nabla u\|^{2}-K^{2}(1-\delta) .
\end{aligned}
$$

Use the inequality (64) in the estimate (63) above and combine the $\int\|\nabla u\|^{2}$ terms on the left hand side. This results in:

$$
\int_{\bar{M}_{\alpha}}\|\nabla u\|^{2} \geq \frac{E_{M S} \delta}{2}-\frac{3 K^{2}(1-\delta)}{2} .
$$

In order to complete the proof of the theorem, the quantity $\delta$ must be chosen between zero and one to make both bounds (62) and (65) positive. This can be accomplished by choosing $\delta$ near enough to 1 to make the negative term in (65) strictly smaller than the positive term.

\section{Norms.}

5.3. Injectivity Estimate for $\Delta_{\bar{M}_{\alpha}}$ in the Weighted Schauder

The next step in the proof of the injectivity estimate is to combine the eigenvalue estimate of the previous section with the elliptic estimate for $\Delta_{\bar{M}_{\alpha}}$ and derive equation (56) in the Schauder $C_{\rho}^{k, \beta}$ norms on $\bar{M}_{\alpha}$. The tool which allows the two estimates to be combined is the De Giorgi Nash inequality. This inequality will make many appearances in the sequel.

FACT: Let $u \in H^{1}\left(\bar{M}_{\alpha}\right)$ be a weak solution of the equation $\Delta_{\bar{M}_{\alpha}} u=f$ where $f \in L^{q / 2}\left(\bar{M}_{\alpha}\right)$ for some $q>n$. Furthermore, suppose $u$ satisfies Neumann boundary conditions. Then there is a constant $C_{D G N}$ depending only on $n$ and $\delta$ so that for every $p \geq 1$,

$$
|u|_{0, \Omega^{\prime}} \leq C_{D G N}\left((\operatorname{Vol}(\Omega))^{-1 / p}\|u\|_{L^{p}(\Omega)}+(\operatorname{Vol}(\Omega))^{2 / n-2 / q}\|f\|_{L^{q / 2}(\Omega)}\right),
$$

where $\Omega$ is any subset of $\bar{M}_{\alpha}$ and $\Omega^{\prime}$ is any subset of the interior of $\Omega$. The reader should consult [19], [20] or [22] for more details.

The constant in the De Giorgi Nash inequality is independent of $\alpha$ because it depends on the metric of $\bar{M}_{\alpha}$ only through the constant appearing in the Michael-Simon inequality (59), and this quantity is independent of $\alpha$. 
The estimate thus provides the essential link between the $\alpha$-independent $L^{2}$ injectivity estimate for $\Delta_{\bar{M}_{\alpha}}$ derived in the previous section and the required $C^{k, \beta}$ estimates.

Proposition 35. If $\alpha \leq A_{4}$, then there exists a constant $C$ independent of $\alpha$ so that

$$
|u|_{0, \bar{M}_{\alpha}} \leq C \varepsilon^{-2 \beta}\left|\rho^{2} \Delta_{\bar{M}_{\alpha}} u\right|_{0, \bar{M}_{\alpha}}
$$

for any function $u \in \mathcal{B}_{1, \alpha}$.

Proof. Suppose that the proposition is false. Then there exists a sequence of parameters $\alpha_{j} \rightarrow 0$ (thus also a sequence of weights $\rho_{j}$ and scales $\varepsilon_{j}$ ) and a sequence of functions $u_{j} \in \mathcal{B}_{1, \alpha_{j}}$, normalized so that $\left|u_{j}\right|_{0, \bar{M}_{\alpha_{j}}}=1$, that satisfy the following inequality:

$$
\varepsilon_{j}^{-2 \beta}\left|\rho_{j}^{2} \Delta_{\bar{M}_{\alpha_{j}}} u_{j}\right|_{0, \bar{M}_{\alpha_{j}}} \leq \frac{1}{j}
$$

for each $j$. Let $x_{j} \in \bar{M}_{\alpha_{j}}$ be a point where $u_{j}\left(x_{j}\right)=1$ and consider a large ball $B_{R}\left(x_{j}\right)$ centered at $x_{j}$ - any $R$ independent of $j$ will do. By the De Giorgi Nash Estimate with $p=2$ and some $q>n$ that will be specified later,

$$
1=\left|u_{j}\right|_{0, B_{\theta R}\left(x_{j}\right)} \leq C\left(\left\|u_{j}\right\|_{L^{2}\left(\bar{M}_{\alpha_{j}}\right)}+\left\|\Delta_{\bar{M}_{\alpha_{j}}} u_{j}\right\|_{L^{q / 2}\left(\bar{M}_{\alpha_{j}}\right)}\right),
$$

where $C$ depends on $C_{D G N}$ and $R$. Next, apply the second eigenvalue estimate along with Green's identity to obtain

$$
\int_{\bar{M}_{\alpha_{j}}} u_{j}^{2} \leq C \int_{\bar{M}_{\alpha_{j}}}\left|u_{j} \cdot \Delta_{\bar{M}_{\alpha_{j}}} u_{j}\right| \leq C\left\|\Delta_{\bar{M}_{\alpha_{j}}} u_{j}\right\|_{L^{1}\left(\bar{M}_{\alpha_{j}}\right)},
$$

using the normalization of $u_{j}$. Substituting the result of equation (69) into (68) and applying Hölder's inequality yields

$$
1 \leq C\left\|\Delta_{\bar{M}_{\alpha_{j}}} u_{j}\right\|_{L^{q / 2}\left(\bar{M}_{\alpha_{j}}\right)} \cdot
$$

This will lead to a contradiction if it can be shown that the right hand side of inequality (70) goes to zero as $j \rightarrow \infty$. Note that in dimension $n=3$, the fraction $q / 2$ can be less than 2 . Thus using the straightforward estimate $\left\|u_{j}\right\|_{L^{2}\left(\bar{M}_{\alpha_{j}}\right)} \leq C\left\|\Delta_{\bar{M}_{\alpha_{j}}} u_{j}\right\|_{L^{2}\left(\bar{M}_{\alpha_{j}}\right)}$ in (68) would not lead to the estimate (70). But the $L^{q / 2}$ norm will turn out to be a crucial component of the following argument. 
To proceed, calculate the $L^{q / 2}$ norm of $\Delta_{\bar{M}_{\alpha_{j}}} u_{j}$.

$$
\begin{aligned}
\left\|\Delta_{\bar{M}_{\alpha_{j}}} u_{j}\right\|_{L^{q / 2}\left(\bar{M}_{\alpha_{j}}\right)} & =\left(\int_{\bar{M}_{\alpha_{j}}}\left|\Delta_{\bar{M}_{\alpha_{j}}} u_{j}\right|^{q / 2}\right)^{2 / q} \\
& \leq R^{-2 \beta}\left(\int_{\bar{M}_{\alpha_{j}}} \rho_{j}^{-q(1-\beta)}\left|\varepsilon_{j}^{-2 \beta} \rho_{j}^{2} \Delta_{\bar{M}_{\alpha_{j}}} u_{j}\right|^{q / 2}\right)^{2 / q} \\
& \leq \frac{C}{j}\left\|\rho_{j}^{-1}\right\|_{L^{q(1-\beta)}\left(\bar{M}_{\alpha_{j}}\right)}^{2(1-\beta)}
\end{aligned}
$$

using the fact that each $\rho_{j}$ is everywhere bounded below by $R \varepsilon_{j}$. By Property 5 of the weight function, $\left\|\rho_{j}^{-1}\right\|_{L^{q(1-\beta)}\left(\bar{M}_{\alpha_{j}}\right)}$ is bounded above independently of $j$ whenever $q<\frac{n}{(1-\beta)}$. Since $n<\frac{n}{1-\beta}$, there is such a choice of $q$ compatible with the requirement $q>n$ that can be made in equation (70). Therefore, the quantity in the right hand side of (70) can be made to approach zero.

Given the result of the previous proposition, it is now an easy matter to transform the elliptic estimate of Theorem 31 into a true injectivity estimate for the operator $\Delta_{\bar{M}_{\alpha}}$ on the space of functions $\mathcal{B}_{1, \alpha}$.

Proposition 36. Suppose $\alpha \leq A_{4}$. Then there is a constant $C$ independent of $\alpha$ so that the estimate

$$
|u|_{C_{\rho}^{2, \beta}\left(\bar{M}_{\alpha}\right)} \leq C \varepsilon^{-2 \beta}\left|\rho^{2} \Delta_{\bar{M}_{\alpha}} u\right|_{C_{\rho}^{0, \beta}\left(\bar{M}_{\alpha}\right)}
$$

holds for any function $u \in \mathcal{B}_{1, \alpha}$.

Proof. The global elliptic estimate of Theorem 31 implies that the function $u$ satisfies:

$$
|u|_{C_{\rho}^{2, \beta}\left(\bar{M}_{\alpha}\right)} \leq C_{E l l}\left(\varepsilon^{-2 \beta}\left|\rho^{2} \Delta_{\bar{M}_{\alpha}} u\right|_{C_{\rho}^{0, \beta}\left(\bar{M}_{\alpha}\right)}+|u|_{0, \bar{M}_{\alpha}}\right) .
$$

The $|u|_{0, \bar{M}_{\alpha}}$ term can be controlled as in Proposition 35 and yields the desired estimate.

\subsection{Injectivity Estimate for $P_{\alpha}$ in the Weighted Schauder Norms.}

The key to finding the injectivity estimate (57) for the operator $P_{\alpha}$ is that $\cos \left(\theta_{\bar{M}_{\alpha}}\right) \approx 1$ and $\sin \left(\theta_{\bar{M}_{\alpha}}\right) \approx 0$ when $\alpha$ is sufficiently small. 
Proposition 37. There is a number $A_{5}$ with $0<A_{5} \leq A_{4}$ and a constant $C>0$ independent of $\alpha$ so that if $\alpha \leq A_{5}$, then

$$
|u|_{C_{\rho}^{2, \beta}\left(\bar{M}_{\alpha}\right)} \leq C \varepsilon^{-2 \beta}\left|\rho^{2} P_{\alpha} u\right|_{C_{\rho}^{0, \beta}\left(\bar{M}_{\alpha}\right)}
$$

for any $u \in \mathcal{B}_{1, \alpha}$. Here, $\theta_{\bar{M}_{\alpha}}$ is the angle function of $\bar{M}_{\alpha}$ and $\vec{H}_{\bar{M}_{\alpha}}$ is its mean curvature vector.

Proof. Start with the right hand side of equation (72).

$$
\left|\rho^{2} P_{\alpha} u\right| \geq\left|\rho^{2} \cos \left(\theta_{\bar{M}_{\alpha}}\right) \Delta_{\bar{M}_{\alpha}} u\right|_{C_{\rho}^{0, \beta}\left(\bar{M}_{\alpha}\right)}-\left|\rho^{2} \sin \left(\theta_{\bar{M}_{\alpha}}\right)\left\langle\vec{H}_{\bar{M}_{\alpha}}, \nabla u\right\rangle\right|_{C_{\rho}^{0, \beta}\left(\bar{M}_{\alpha}\right)}
$$

The first term of (73) is easy to deal with by applying the injectivity estimate from Proposition 36 and the properties of the trigonometric functions of $\theta_{\bar{M}_{\alpha}}$ listed in equations (29):

$$
\begin{aligned}
& \left|\rho^{2} \cos \left(\theta_{\bar{M}_{\alpha}}\right) \Delta_{\bar{M}_{\alpha}} u\right|_{C_{\rho}^{0, \beta}\left(\bar{M}_{\alpha}\right)} \\
& =\left|\rho^{2} \cos \left(\theta_{\bar{M}_{\alpha}}\right) \Delta_{\bar{M}_{\alpha}} u\right|_{0, \bar{M}_{\alpha}}+\left[\rho^{2+\beta} \cos \left(\theta_{\bar{M}_{\alpha}}\right) \Delta_{\bar{M}_{\alpha}} u\right]_{\beta, \bar{M}_{\alpha}} \\
& \geq\left|\rho^{2} \Delta_{\bar{M}_{\alpha}}\right|_{0, \bar{M}_{\alpha}} \cdot\left|\cos \left(\theta_{\bar{M}_{\alpha}}\right)\right|_{0, \bar{M}_{\alpha}}+\left[\rho^{2+\beta} \Delta_{\bar{M}_{\alpha}} u\right]_{\beta, \bar{M}_{\alpha}} \cdot\left|\cos \left(\theta_{\bar{M}_{\alpha}}\right)\right|_{0, \bar{M}_{\alpha}} \\
& \quad \quad-\left|\rho^{2} \Delta_{\bar{M}_{\alpha}} u\right|_{0, \bar{M}_{\alpha}} \cdot\left|\rho^{\beta}\right|_{0, \bar{M}_{\alpha}} \cdot\left[\cos \left(\theta_{\bar{M}_{\alpha}}\right)\right]_{\beta, \bar{M}_{\alpha}} \\
& \geq C\left(1-\alpha^{1-\beta}\right)\left|\rho^{2} \Delta_{\bar{M}_{\alpha}} u\right|_{C_{\rho}^{0, \beta}\left(\bar{M}_{\alpha}\right)} \\
& \geq C \varepsilon^{2 \beta}|u|_{C_{\rho}^{2, \beta}\left(\bar{M}_{\alpha}\right)},
\end{aligned}
$$

for some constant $C$ independent of $\alpha$ when $\alpha$ is sufficiently small. The second term of (73) can be estimated from below using inequality (44) from Proposition 30 and results in

$$
\left|\rho^{2} \cos \left(\theta_{\bar{M}_{\alpha}}\right) \Delta_{\bar{M}_{\alpha}} u+\sin \left(\theta_{\bar{M}_{\alpha}}\right) \vec{H}_{\bar{M}_{\alpha}} \cdot \nabla u\right|_{C_{\rho}^{0, \beta}\left(\bar{M}_{\alpha}\right)} \geq C \varepsilon^{2 \beta}\left(1-\alpha \varepsilon^{-2 \beta}\right)|u|_{C_{\rho}^{2, \beta}\left(\bar{M}_{\alpha}\right)} .
$$

This gives the desired estimate when $\alpha$ is sufficiently small.

\subsection{Properties of the First Eigenfunction.}

In order to complete the injectivity component of the proof of the Main Theorem, it remains only to extend the injectivity bounds derived in the previous section to the full linearized deformation operator $(u, a, b) \mapsto P_{\alpha} u+$ $a \psi_{0, \alpha}+b \psi_{1, \alpha}$. Recall that the functions $u$ are taken in the Banach space $\mathcal{B}_{1, \alpha}$ and are thus $L^{2}$-orthogonal to the span of 1 and $S_{\alpha}$, where $S_{\alpha}$ is the 
first non-trivial eigenfunction of $\Delta_{\bar{M}_{\alpha}}$. It will be shown that the injectivity estimate for the full linearized operator is equivalent to finding a lower bound, independent of $\alpha$, on the $L^{2}$ inner products

$$
\int_{\bar{M}_{\alpha}} \psi_{i, \alpha} \quad \text { and } \quad \int_{\bar{M}_{\alpha}} \psi_{i, \alpha} \cdot S_{\alpha}
$$

for $i=1$ and 2. Unfortunately, too little is known about the function $S_{\alpha}$ to accomplish this. The purpose of this section is to derive the necessary properties of $S_{\alpha}$. Begin with the following lemma.

Lemma 38. There is a number $A_{6}$ with $0<A_{6} \leq A_{5}$ and a constant $C>0$ independent of $\alpha$ so that if $\alpha \leq A_{6}$, then the function $S_{\alpha}$ satisfies $\left|S_{\alpha}\right|_{0, \bar{M}_{\alpha}} \leq C$.

Proof. Let $x \in \bar{M}_{\alpha}$ be a point where $S_{\alpha}$ is maximal and let $B_{R}(x)$ be any ball about $x$ with radius independent of $\alpha$. Apply the De Giorgi Nash inequality with $p=2$ and any $q>n$ to $S_{\alpha}$ to $S_{\alpha}$ :

$$
\begin{aligned}
\left|S_{\alpha}\right|_{0, \bar{M}_{\alpha}} & =\left|S_{\alpha}\right|_{0, B_{R}(x)} \\
& \leq C\left(\left\|\Delta_{\bar{M}_{\alpha}} S_{\alpha}\right\|_{L^{q / 2}\left(\bar{M}_{\alpha}\right)}+\left\|S_{\alpha}\right\|_{L^{2}\left(\bar{M}_{\alpha}\right)}\right) \\
& \leq C\left(\nu_{1}\left|S_{\alpha}\right|_{0, \bar{M}_{\alpha}}+1\right) .
\end{aligned}
$$

Recall that $\nu_{1} \leq C \alpha^{n-2}$; thus if $\alpha$ is sufficiently small, the $\left|S_{\alpha}\right|_{0, \bar{M}_{\alpha}}$ terms can be grouped together in (76), and lead to the desired estimate.

The remaining properties of $S_{\alpha}$ will be derived by first approximating this function and then deriving these properties from the approximation. As in Proposition 20 of Section 3.4, let $\sigma$ be a function which is equal to 1 in $M_{1}^{\prime}$, equal to -1 in $M_{2}^{\prime}$, and which interpolates smoothly between 1 and -1 in the neck region of $\bar{M}_{\alpha}$. Furthermore, choose $\sigma$ so that the derivative bounds $\|\nabla \sigma\|+\alpha\left\|\nabla^{2} \sigma\right\| \leq C \alpha^{-1}$ are satisfied within the neck region. This function can not be in $\mathcal{B}_{1, \alpha}$ because of the following result.

Lemma 39. The quantity $\int_{\bar{M}_{\alpha}} \sigma \cdot S_{\alpha}$ is bounded below by a positive constant independent of $\alpha$.

Proof. Assume that the lemma is false. Then there is a sequence $\alpha_{j} \rightarrow 0$ and a sequence of interpolating functions $\sigma_{j}$ as above so that $\int_{\bar{M}_{\alpha_{j}}} \sigma_{j} \cdot S_{\alpha_{j}} \rightarrow$ 
0 . Let $u_{j}=\sigma_{j}-\left\langle\sigma_{j}, 1\right\rangle / \operatorname{Vol}\left(\bar{M}_{\alpha_{j}}\right)-\left\langle\sigma_{j}, S_{\alpha_{j}}\right\rangle S_{\alpha_{j}}$, using the abbreviation $\langle u, v\rangle=\int_{\bar{M}_{\alpha_{j}}} u \cdot v$. Now, $\left\langle u_{j}, 1\right\rangle=\left\langle u_{j}, S_{\alpha_{j}}\right\rangle=0$ so $u_{j} \in \mathcal{B}_{1, \alpha_{j}}$. Consequently, the $\alpha_{j}$-independent second eigenvalue estimate applies to $u_{j}$ to give

$$
\int_{\bar{M}_{\alpha_{j}}}\left\|\nabla u_{j}\right\|^{2} \geq C_{2} \int_{\bar{M}_{\alpha_{j}}} u_{j}^{2}
$$

which implies

$$
\left\|\nabla \sigma_{j}\right\|_{L^{2}\left(\bar{M}_{\alpha_{j}}\right)}^{2}+\left\langle\sigma_{j}, S_{\alpha_{j}}\right\rangle^{2} \geq C\left(\left\|u_{j}\right\|_{L^{2}\left(\bar{M}_{\alpha_{j}}\right)}^{2}-\left\langle\sigma_{j}, 1\right\rangle^{2}\right)
$$

using orthogonality properties of the functions 1 and $S_{\alpha_{j}}$ and Green's identity. By construction of $\sigma_{j}$, the right hand side of (77) is bounded below by a constant independent of $j$, whereas the $\left\langle\sigma_{j}, S_{\alpha_{j}}\right\rangle$ term goes to zero with $j$ by assumption and $\left\|\nabla \sigma_{j}\right\|_{L^{2}\left(\bar{M}_{\alpha_{j}}\right)}^{2} \leq C \alpha_{j}^{n-2} \rightarrow 0$ as calculated in Proposition 20. This contradiction proves the lemma.

Since the quantity $\left\langle\sigma, S_{\alpha}\right\rangle$ is positive, one can define the approximate first eigenfunction $\bar{S}_{\alpha}$ by

$$
\bar{S}_{\alpha}=\frac{\sigma-\langle\sigma, 1\rangle \operatorname{Vol}\left(\bar{M}_{\alpha}\right)^{-1}}{\left\langle\sigma, S_{\alpha}\right\rangle} .
$$

Note that $\left\langle\bar{S}_{\alpha}, 1\right\rangle=0$ and $\left\langle\bar{S}_{\alpha}, S_{\alpha}\right\rangle=1$. The two properties of $S_{\alpha}$ needed to estimate the inner products $(75)$ will be deduced from the $C^{0}$ difference between $S_{\alpha}$ and $\bar{S}_{\alpha}$.

Proposition 40. Suppose $\alpha \leq A_{6}$. Then the approximate first eigenfunction $\bar{S}_{\alpha}$ satisfies the estimate

$$
\left|\bar{S}_{\alpha}-S_{\alpha}\right|_{0, \bar{M}_{\alpha}} \leq C \alpha^{(n-2) / 2}
$$

where $C$ is a constant independent of $\alpha$.

Proof. Let $x \in \bar{M}_{\alpha}$ be a point where $\bar{S}_{\alpha}-S_{\alpha}$ is maximal and let $B_{R}(x)$ be any ball about $x$ with radius independent of $\alpha$. The De Giorgi Nash estimate with $p=2$ and any $q>n$ implies that

$$
\left|\bar{S}_{\alpha}-S_{\alpha}\right|_{0, B_{R}(x)} \leq C\left(\left\|\bar{S}_{\alpha}-S_{\alpha}\right\|_{L^{2}\left(\bar{M}_{\alpha}\right)}+\left\|\Delta_{\bar{M}_{\alpha}} \bar{S}_{\alpha}-\Delta_{\bar{M}_{\alpha}} S_{\alpha}\right\|_{L^{q / 2}\left(\bar{M}_{\alpha}\right)}\right) .
$$


Estimate each term on the right hand side of (80) in turn. First, by construction of $\bar{S}_{\alpha}$, the difference $\bar{S}_{\alpha}-S_{\alpha}$ is orthogonal to 1 and $S_{\alpha}$; thus the min-max characterization of the second eigenvalue as well as the triangle inequality yields

$$
\begin{aligned}
\left\|\bar{S}_{\alpha}-S_{\alpha}\right\|_{L^{2}\left(\bar{M}_{\alpha}\right)} & \leq C\left(\left\|\nabla \bar{S}_{\alpha}\right\|_{L^{2}\left(\bar{M}_{\alpha}\right)}+\left\|\nabla S_{\alpha}\right\|_{L^{2}\left(\bar{M}_{\alpha}\right)}\right) \\
& \left.\leq C\left\|\nabla \bar{S}_{\alpha}\right\|_{L^{2}\left(\bar{M}_{\alpha}\right)}+\left\|S_{\alpha} \cdot \Delta S_{\alpha}\right\|_{L^{2}\left(\bar{M}_{\alpha}\right)}\right) \\
& \leq C \alpha^{(n-2) / 2}, \quad \text { (by Green's identity) }
\end{aligned}
$$

by the derivative bounds on $\bar{S}_{\alpha}$ and the fact that $S_{\alpha}$ is a normalized eigenfunction of $\Delta_{\bar{M}_{\alpha}}$ with small eigenvalue proportional to $\alpha^{n-2}$. Next,

$$
\begin{aligned}
\left\|\Delta_{\bar{M}_{\alpha}} \bar{S}_{\alpha}-\Delta_{\bar{M}_{\alpha}} S_{\alpha}\right\|_{L^{q / 2}\left(\bar{M}_{\alpha}\right)} & \leq\left\|\Delta_{\bar{M}_{\alpha}} \bar{S}_{\alpha}\right\|_{L^{q / 2}\left(\bar{M}_{\alpha}\right)}+\left\|\Delta_{\bar{M}_{\alpha}} S_{\alpha}\right\|_{L^{q / 2}\left(\bar{M}_{\alpha}\right)} \\
& \leq C \alpha^{-1+n q / 2}
\end{aligned}
$$

using the derivative bounds of $\bar{S}_{\alpha}$ and the first eigenvalue estimate once again. Combining (81) and (82) in (80) yields the desired estimate.

This more specific information about the eigenfunction $S_{\alpha}$ makes it possible now to deduce the lower bounds (75). The most important of these, and the only one which needs to be seen explicitly, is the following one.

Proposition 41. There is a number $A_{7}$ with $0<A_{7} \leq A_{6}$ and a constant $C>0$ which is independent of $\alpha$ so that if $\alpha \leq A_{7}$ then the function $\psi_{1, \alpha}$ satisfies the inequality $\left|\left\langle\psi_{1, \alpha}, S_{\alpha}\right\rangle\right| \geq C$.

Proof. Choose $\alpha \leq A_{6}$ so that all the estimates of the previous sections are valid. Using integration by parts, the Neumann condition and the definition of $v_{e}$ given in equation (37), one deduces

$$
\begin{aligned}
-\left\langle\psi_{1, \alpha}, S_{\alpha}\right\rangle & =\left\langle\Delta_{\bar{M}_{\alpha}} v_{e}, S_{\alpha}\right\rangle \\
& =\int_{\partial \bar{M}_{\alpha}} S_{\alpha} \frac{\partial v_{e}}{\partial n}-\nu_{1}\left\langle v_{e}, S_{\alpha}\right\rangle \\
& =\int_{\partial \bar{M}_{\alpha}} S_{\alpha} v-\nu_{1}\left\langle v_{e}, S_{\alpha}\right\rangle .
\end{aligned}
$$

The properties of $S_{\alpha}$ that have been deduced from the approximation $\bar{S}_{\alpha}$ will be used to estimate both these terms. First, by the estimate of $\nu_{1}$,

$$
\nu_{1}\left\langle v_{e}, S_{\alpha}\right\rangle \leq C_{1} \alpha^{n-2}
$$


where $C$ is independent of $\alpha$ because the $L^{2}$ norm of $v_{e}$ depends only on the geometry of $\bar{M}_{\alpha}$ near its boundary. Next,

$$
\int_{\partial \bar{M}_{\alpha}} S_{\alpha} v \geq \int_{\partial \bar{M}_{\alpha}} \bar{S}_{\alpha} v-\int_{\partial \bar{M}_{\alpha}}\left|S_{\alpha}-\bar{S}_{\alpha}\right||v| \geq \frac{2}{\left\langle\sigma, S_{\alpha}\right\rangle}-C \alpha^{(n-2) / 2},
$$

using the supremum norm estimate of $\left|\bar{S}_{\alpha}-S_{\alpha}\right|$ at the boundary and the fact that the boundary values of $\bar{S}_{\alpha}$ are known exactly. In Lemma 39, it was shown that the quantity $\left\langle\sigma, S_{\alpha}\right\rangle$ is bounded below by a constant independent of $\alpha$. Thus combining the upper bound (84) with the lower bound (85) in inequality (83) yields the desired result when $\alpha$ is sufficiently small.

\subsection{The Full Injectivity Estimate for $\mathrm{D} F_{\alpha}(0,0,0)$.}

The groundwork for the full injectivity estimate for the operator $\mathrm{D} F_{\alpha}(0,0,0)$ has now been laid and it is possible to prove the following theorem.

Theorem 42. If $\alpha \leq A_{7}$, then there is a geometric constant $C$ independent of $\alpha$ so that the following estimate is valid:

$$
\left(|u|_{C_{\rho}^{2, \beta}\left(\bar{M}_{\alpha}\right)}^{2}+a^{2}+b^{2}\right)^{1 / 2} \leq C \varepsilon^{-2 \beta}\left|\rho^{2}\left(P_{\alpha} u+a \psi_{0, \alpha}+b \psi_{1, \alpha}\right)\right|_{C_{\rho}^{0, \beta}\left(\bar{M}_{\alpha}\right)}
$$

for any $(u, a, b) \in \mathcal{B}_{1, \alpha} \times \mathbf{R}^{2}$. Consequently, $C_{L}(\alpha)=C \varepsilon^{2 \beta}$

Proof. Suppose that the theorem is false. Then there is a sequence of parameters $\alpha_{j} \rightarrow 0$ (thus also a sequence of scales $\varepsilon_{j}$ and weight functions $\left.\rho_{j}\right)$ and a sequence of elements $\left(u_{j}, a_{j}, b_{j}\right) \in \mathcal{B}_{1, \alpha_{j}}$, normalized so that $\left\|\left(u_{j}, a_{j}, b_{j}\right)\right\|_{C_{\rho_{j}}^{2, \beta}\left(\bar{M}_{\alpha_{j}}\right) \times \mathbf{R}^{2}}=1$ for every $j$, which satisfy the estimates

$$
\varepsilon_{j}^{-2 \beta}\left|\rho_{j}^{2}\left(P_{\alpha_{j}} u_{j}+a_{j} \psi_{0, \alpha_{j}}+b_{j} \psi_{1, \alpha_{j}}\right)\right|_{C_{\rho_{j}}^{0, \beta}\left(\bar{M}_{\alpha_{j}}\right)} \leq \frac{1}{j} .
$$

It will be shown that $\left(u_{j}, a_{j}, b_{j}\right) \rightarrow(0,0,0)$, contradicting the normalization.

The first step towards establishing the contradiction is to show that the sequences $a_{j}$ and $b_{j}$ tend towards zero as $j \rightarrow \infty$. Begin by integrating both sides of equation (87) over $\bar{M}_{\alpha_{j}}$ and estimating terms:

$$
\begin{gathered}
\int_{\bar{M}_{\alpha_{j}}} \frac{\varepsilon_{j}^{2 \beta}}{j} \geq\left|a_{j}\right| \int_{\bar{M}_{\alpha_{j}}} \rho_{j}^{2} \psi_{0, \alpha_{j}}-\left|b_{j}\right|\left|\int_{\bar{M}_{\alpha_{j}}} \rho_{j}^{2} \psi_{1, \alpha_{j}}\right|-\left|\int_{\bar{M}_{\alpha_{j}}} \rho_{j}^{2} \cos \left(\theta_{\bar{M}_{\alpha_{j}}}\right) \Delta_{\bar{M}_{\alpha_{j}}} u_{j}\right| \\
-\left|\int_{\bar{M}_{\alpha_{j}}} \rho_{j}^{2} \sin \left(\theta_{\bar{M}_{\alpha_{j}}}\right)\left\langle\vec{H}_{\bar{M}_{\alpha_{j}}} \nabla u_{j}\right\rangle\right|
\end{gathered}
$$


The first integral in equation (88) is bounded below according since $\rho=R$ and $\psi_{0, \alpha_{j}}=1$ in $M_{1}^{\prime} \cup M_{2}^{\prime}$. The second integral vanishes because $\rho_{j}$ is constant in the support of $\psi_{1, \alpha_{j}}$ which occurs only near the boundary:

$$
\begin{aligned}
\int_{\bar{M}_{\alpha_{j}}} \rho_{j}^{2} \psi_{1, \alpha_{j}} & =-R^{2} \int_{\bar{M}_{\alpha_{j}}} \Delta_{\bar{M}_{\alpha_{j}}} v_{e} \\
& =R^{2} \int_{\partial \bar{M}_{\alpha_{j}}} v \\
& =0
\end{aligned}
$$

by the definition of $v$.

For the third integral in equation (88), use the orthogonality of $\Delta_{\bar{M}_{\alpha_{j}}} u_{j}$ to the constant function $R^{2}$ to estimate

$$
\begin{gathered}
\left|\int_{\bar{M}_{\alpha_{j}}} \rho_{j}^{2} \cos \left(\theta_{\bar{M}_{\alpha_{j}}}\right) \Delta_{\bar{M}_{\alpha_{j}}} u_{j}\right|=\left|\int_{\bar{M}_{\alpha_{j}}}\left(\rho_{j}^{2} \cos \left(\theta_{\bar{M}_{\alpha_{j}}}\right)-R^{2}\right) \Delta_{\bar{M}_{\alpha_{j}}} u_{j}\right| \\
\leq C\left\|\rho_{j}^{2}-R^{2}\right\|_{L^{2}\left(\bar{M}_{\alpha_{j}}\right)}\left\|\Delta_{\bar{M}_{\alpha_{j}}} u\right\|_{L^{2}\left(\bar{M}_{\alpha_{j}}\right)} \\
\text { (using the bounds on } \left.\theta_{\bar{M}_{\alpha_{j}}}\right) \\
\leq C \varepsilon_{j}^{n \beta}
\end{gathered}
$$

because of the fact that $\rho_{j}$ differs from $R$ only in a ball of radius $\varepsilon_{j}^{\beta}$. The second eigenvalue estimate as well as the normalization of $u_{j}$ have been used to control the $\left\|\Delta_{\bar{M}_{\alpha_{j}}} u\right\|_{L^{2}\left(\bar{M}_{\alpha_{j}}\right)}$ term. Finally, the last integral in (88) is bounded above by $C \alpha_{j}$ because of the bounds on $\theta_{\bar{M}_{\alpha_{j}}}$ from (29) and on the mean curvature from Proposition 15. Taking this fact along with equations (90) and (89) together with equation (88) and rearranging terms yields an estimate of the form

$$
\left|a_{j}\right| \leq C\left(\frac{\varepsilon_{j}^{2 \beta}}{j}+\varepsilon_{j}^{n \beta}+\alpha_{j}\right)
$$

which shows that $a_{j} \rightarrow 0$.

Next, multiply both sides of equation (87) by $S_{\alpha_{j}}$, integrate over $\bar{M}_{\alpha_{j}}$, and use the orthogonality of $\Delta_{\bar{M}_{\alpha_{j}}} u_{j}$ to the eigenfunction $S_{\alpha_{j}}$ as in (90) to 
obtain

$$
\begin{aligned}
& C \frac{\varepsilon_{j}^{2 \beta}\left|S_{\alpha_{j}}\right|_{0, \bar{M}_{\alpha_{j}}}}{j} \\
& \geq\left|b_{j}\right|\left|\int_{\bar{M}_{\alpha_{j}}} \rho_{j}^{2} \psi_{1, \alpha_{j}} \cdot S_{\alpha_{j}}\right|-\left|\int_{\bar{M}_{\alpha_{j}}}\left(\rho_{j}^{2} \cos \left(\theta_{\bar{M}_{\alpha_{j}}}\right)-R^{2}\right)\left(\Delta_{\bar{M}_{\alpha_{j}}} u_{j}\right) S_{\alpha_{j}}\right| \\
& \quad-\left|\int_{\bar{M}_{\alpha_{j}}} \sin \left(\theta_{\bar{M}_{\alpha_{j}}}\right)\left\langle\vec{H}_{\bar{M}_{\alpha_{j}}}, \nabla u_{j}\right\rangle\right|-\left|a_{j}\right|\left|\int_{\bar{M}_{\alpha_{j}}} \rho_{j}^{2} \psi_{0, \alpha_{j}} \cdot S_{\alpha_{j}}\right| .
\end{aligned}
$$

The $\left|S_{\alpha_{j}}\right|_{0, \bar{M}_{\alpha_{j}}}$ term can be bounded below by using the approximating eigenfunction $\bar{S}_{\alpha_{j}}$. The first integral on the right hand side of equation (92) is bounded below as a result of Lemma 41 and the properties of $\rho$. The second and third integrals can be bounded below exactly as in the analysis of equation (90). The last integral can be bounded below using the fact that $\rho_{j}^{2} \psi_{0, \alpha_{j}} \approx 1$ in $M_{1}^{\prime} \cup M_{2}^{\prime}$ and $\int_{\bar{M}_{\alpha_{j}}} S_{\alpha_{j}}=0$. Consequently, equation (92) leads to an estimate of the form

$$
\left|b_{j}\right| \leq C\left(\frac{\varepsilon_{j}^{4 \beta}}{j}+\left|a_{j}\right|+\varepsilon_{j}^{n \beta}+\alpha_{j}\right),
$$

where $C$ is independent of $j$, which shows that the sequence $b_{j} \rightarrow 0$ as well.

The fact that the limits of the sequences $a_{j}$ and $b_{j}$ are zero has two contradictory consequences. On the one hand, the normalization of $\left(u_{j}, a_{j}, b_{j}\right)$ implies that if $j$ is sufficiently large, then $\left|u_{j}\right|_{C_{\rho_{j}}^{2, \beta}\left(\bar{M}_{\alpha_{j}}\right)} \geq \frac{1}{2}$. On the other hand, the assumption (87) in conjunction with (91) and (93) implies that

$$
\varepsilon_{j}^{-2 \beta}\left|\rho_{j}^{2} \Delta_{\bar{M}_{\alpha_{j}}} u_{j}\right|_{C_{\rho_{j}}^{0, \beta}\left(\bar{M}_{\alpha_{j}}\right)} \longrightarrow 0 .
$$

Thus by the injectivity estimate for $\Delta_{\bar{M}_{\alpha_{j}}}$ in the $C_{\rho_{j}}^{k, \beta}$ norms,

$$
\frac{1}{2} \leq\left|u_{j}\right|_{C_{\rho_{j}}^{2, \beta}\left(\bar{M}_{\alpha_{j}}\right)} \leq C \varepsilon_{j}^{-2 \beta}\left|\rho_{j}^{2} \Delta_{\bar{M}_{\alpha_{j}}} u_{j}\right|_{C_{\rho_{j}}^{0, \beta}\left(\bar{M}_{\alpha_{j}}\right)} \longrightarrow 0 .
$$

This contradiction shows that the assumption (87) must be false, thereby completing the proof of the theorem.

\subsection{Surjectivity of the Linearized Operator.}

The preceding result concludes the lengthy proof of the injectivity of the linearized deformation operator $\mathrm{DF}_{\alpha}(0,0,0)$ and identifies the dependence 
of the injectivity bound on the parameter $\alpha$. The final step in the analysis of the linearized operator is to show surjectivity. But given all the work that has been done so far, this is now a fairly trivial matter.

Theorem 43. If $\alpha \leq A_{7}$, then the operator $\mathrm{DF}(0,0,0): \mathcal{B}_{1, \alpha} \times R^{2} \longrightarrow$ $C_{\rho}^{0, \beta}\left(\bar{M}_{\alpha}\right)$ is surjective.

Proof. Recall that when $\alpha$ is sufficiently small, $\mathrm{D} F_{\alpha}(0,0,0)$ is a small perturbation of the operator

$$
(u, a, b) \mapsto \Delta_{\bar{M}_{\alpha}} u+a \psi_{0, \alpha}+b \psi_{1, \alpha} .
$$

Thus by standard elliptic theory, the surjectivity of $\mathrm{D} F_{\alpha}(0,0,0)$ is equivalent to the surjectivity of the operator in (94).

Let $f$ be any function in $C_{\rho}^{0, \beta}\left(\bar{M}_{\alpha}\right)$. To show surjectivity, one must solve the equation

$$
\Delta_{\bar{M}_{\alpha}} u=a \psi_{0, \alpha}+b \psi_{1, \alpha}-f
$$

for $(u, a, b) \in \mathcal{B}_{1, \alpha} \times \mathbf{R}^{2}$. By the self-adjointness of the Laplacian, equation (95) can be solved for $u \in \mathcal{B}_{1, \alpha}$ if and only if the right hand side of the equation is orthogonal to both the functions 1 and $S_{\alpha}$. Thus $a$ and $b$ must be chosen to satisfy the system of equations:

$$
\begin{gathered}
a \int_{\bar{M}_{\alpha}} \psi_{0, \alpha}+b \int_{\bar{M}_{\alpha}} \psi_{1, \alpha}=\int_{\bar{M}_{\alpha}} f \\
a \int_{\bar{M}_{\alpha}} \psi_{0, \alpha} S_{\alpha}+b \int_{\bar{M}_{\alpha}} \psi_{1, \alpha} S_{\alpha}=\int_{\bar{M}_{\alpha}} f S_{\alpha}
\end{gathered}
$$

in order to solve (95). But recall once again that $\int_{\bar{M}_{\alpha}} \psi_{1, \alpha}=\int_{\partial \bar{M}_{\alpha}} v=0$ and that $\int_{\bar{M}_{\alpha}} \psi_{0, \alpha}$ is strictly positive. Thus,

$$
a=\left(\int_{\bar{M}_{\alpha}} f\right)\left(\int_{\bar{M}_{\alpha}} \psi_{0, \alpha}\right)^{-1} .
$$

This value of $a$ can now be substituted into the second of the two equations in (96) above and because $\int_{\bar{M}_{\alpha}} \psi_{1, \alpha} S_{\alpha}$ is also strictly positive according to Proposition (41), $b$ can be found as well.

The integrability conditions for equation (95) can thus be satisfied by a suitable choice of $a$ and $b$. Consequently, a function $u \in \mathcal{B}_{1, \alpha}$ satisfying (95) exists. The regularity of $u$ follows from elliptic regularity theory for the operator $\Delta_{\bar{M}_{\alpha}}$ and the fact that $f, \psi_{0, \alpha}$ and $\psi_{1, \alpha}$ are at least $C^{0, \beta}$. 


\section{Solving the Nonlinear Problem.}

\subsection{The Nonlinear Estimate.}

The remaining components of the proof of the Main Theorem are to bound the nonlinearities of $F_{\alpha}$ and to estimate the size of $F_{\alpha}(0,0,0)$ according to the outline presented in Section 2.1. The difference

$$
\begin{aligned}
& \left|\rho^{2}\left(\left(\mathrm{D} F_{\alpha}(H, \theta, B)-\mathrm{D} F_{\alpha}(0,0,0)\right)(u, a, b)\right)\right|_{C_{\rho}^{0, \beta}\left(\bar{M}_{\alpha}\right)} \\
& \quad \leq C_{N}(\alpha)\|(H, \theta, B)\|_{C_{\rho}^{2, \beta}\left(\bar{M}_{\alpha}\right) \times \mathbf{R}^{2}}\|(u, a, b)\|_{C_{\rho}^{2, \beta}\left(\bar{M}_{\alpha}\right) \times \mathbf{R}^{2}}
\end{aligned}
$$

will be estimated using scaling techniques, and starts with a lemma concerning the behaviour of the Hamiltonian flow under rescaling

Lemma 44. Let $H: \mathbf{R}^{2 n} \longrightarrow \mathbf{R}$ be a $C^{2, \beta}$ function and suppose $\phi_{H}^{t}$ denotes the Hamiltonian flow associated to $H$. If $\sigma_{\varepsilon}: \mathbf{R}^{2 n} \longrightarrow \mathbf{R}^{2 n}$ is the diffeomorphism given by $\sigma_{\varepsilon}(x)=\varepsilon x$ then

$$
\sigma_{\varepsilon}^{-1} \circ \phi_{H}^{t} \circ \sigma_{\varepsilon}=\phi_{H^{\varepsilon}}^{t}
$$

where $H^{\varepsilon}$ is the function $\varepsilon^{-2} H \circ \sigma_{\varepsilon}$.

Proof. . The proof is simply a matter of differentiating both sides in $t$ and comparing the results.

This fact about the Hamiltonian flow makes it possible to deduce the dependence of $C_{N}(\alpha)$ on $\alpha$.

Proposition 45. Suppose $\alpha \leq A_{7}$. Then the linearization of the operator $F_{\alpha}$ at the point $(H, \theta, B)$ in $\mathcal{B}_{1, \alpha} \times \mathbf{R}^{2}$ satisfies the estimate:

$$
\begin{aligned}
& \left|\rho^{2}\left(\mathrm{D} F_{\alpha}(H, \theta, B)-\mathrm{D} F_{\alpha}(0,0,0)\right)(u, a, b)\right|_{C_{\rho}^{0, \beta}\left(\bar{M}_{\alpha}\right)} \\
& \quad \leq C_{N} \varepsilon^{-2-2 \beta}\|(H, \theta, B)\|_{C_{\rho}^{2, \beta}\left(\bar{M}_{\alpha}\right) \times \mathbf{R}^{2}}\|(u, a, b)\|_{C_{\rho}^{2, \beta}\left(\bar{M}_{\alpha}\right) \times \mathbf{R}^{2}}
\end{aligned}
$$

for all $(u, a, b) \in \mathcal{B}_{1, \alpha} \times \mathbf{R}^{2}$, where $C_{N}$ is a constant independent of $\alpha$.

Proof. Choose $x \in \bar{M}_{\alpha}$ and let $s=\rho(x)$. Denote by $\sigma$ the rescaling given by $\sigma(x)=s x$. Denote by $\bar{h}_{1}$ the rescaled embedding $\sigma^{-1} \circ \bar{h}_{\alpha}$ which embeds the 
submanifold $\frac{1}{s} \bar{M}_{\alpha}$ into $\mathbf{R}^{2 n}$. Furthermore, let $\mathrm{Vol}_{1}$ denote the volume form of this embedding and $\langle\cdot, \cdot\rangle_{1}$ its induced metric. Recall that $F_{\alpha}(H, \theta, B)$ is a $C^{0, \beta}$ function of $\bar{M}_{\alpha}$ and so it can be evaluated at $x$. This leads to the calculation:

$$
\begin{aligned}
& F_{\alpha}(H, \theta, B)(x) \\
& =\left\langle\left(\phi_{v_{e}}^{B} \circ \phi_{H_{e}}^{1} \circ \bar{h}_{1}\right)^{*} \sigma^{*} \operatorname{Im}\left(\mathrm{e}^{\mathrm{i} \theta} \mathrm{d} z\right), \operatorname{Vol}_{\bar{M}_{\alpha}}\right\rangle_{\bar{M}_{\alpha}}(x) \\
& =\left\langle\left(\left(\sigma^{-1} \circ \phi_{v_{e}}^{B} \circ \sigma\right) \circ\left(\sigma^{-1} \circ \phi_{H_{e}}^{1} \circ \sigma\right) \circ \bar{h}_{1}\right)^{*} \sigma^{*} \mathbf{I m}\left(\mathrm{e}^{\mathrm{i} \theta} \mathrm{d} z\right), \operatorname{Vol}_{\bar{M}_{\alpha}}\right\rangle_{\bar{M}_{\alpha}}(x) \\
& =\left\langle\left(\phi_{v_{e}}^{s^{-1} B} \circ \phi_{H_{e}^{s}}^{1} \circ \bar{h}_{1}\right)^{*} \operatorname{Im}\left(\mathrm{e}^{\mathrm{i} \theta} \mathrm{d} z\right), \operatorname{Vol}_{1}\right\rangle_{1}(\sigma(x)) .
\end{aligned}
$$

The right side of equation (99) can be written as $G\left(H^{s}, \theta, s^{-1} B\right)(x)$, where $G: \mathbf{R}^{2 n} \rightarrow \mathbf{R}$ restricted to the $\bar{M}_{\alpha}$. Since, by definition of $\rho$ the bounds on the induced metric of $\bar{M}_{\alpha}$ are uniform in a ball of radius $\frac{s}{2 K_{\varepsilon}}$ about $x$, the map $G$ is independent of $\alpha$ in this ball. Consequently,

$$
\begin{aligned}
& \left|\rho^{2}\left(\mathrm{D} F_{\alpha}(H, \theta, B)-\mathrm{D} F_{\alpha}(0,0,0)\right)\right|_{0, B_{s / 2 K_{\varepsilon}}(x)} \\
& \leq\left|\rho^{2} \cdot\left(\left.\frac{\mathrm{d} G\left(H^{s}+t u^{s}, \theta+t a, B / s+t b / s\right)}{\mathrm{d} t}\right|_{t=0}-\left.\frac{\mathrm{d} G\left(t u^{s}, t a, t b / s\right)}{\mathrm{d} t}\right|_{t=0}\right)\right|_{0, B_{s / 2 K_{\varepsilon}}(x)} \\
& \leq C s^{2} \cdot\left\|\left(u^{s}, a, s^{-1} b\right)\right\|_{C^{2}\left(B_{1 / 2 K_{\varepsilon}}(x)\right) \times \mathbf{R}^{2}} \cdot\left\|\left(H^{s}, \theta, s^{-1} B\right)\right\|_{C^{2}\left(B_{1 / 2 K_{\varepsilon}}(x)\right) \times \mathbf{R}^{2}},
\end{aligned}
$$

as a result of straightforward continuity bounds on the map $G$ in the rescaled ball $B_{1 / 2 K_{\varepsilon}}(x)$. Simple scaling arguments and the lower bound on $\rho$ then lead to the estimate

$$
\left.\left\|\left(u^{s}, a, s^{-1} b\right)\right\|_{C^{2}\left(B_{1 / 2 K_{\varepsilon}}(x)\right) \times \mathbf{R}^{2}} \leq C \varepsilon^{-2}\|(u, a, b)\|_{C^{2}\left(B_{s} / 2 K_{\varepsilon}\right.}(x)\right) \times \mathbf{R}^{2}
$$

for any $(u, a, b) \in \mathcal{B}_{1, \alpha} \times \mathbf{R}^{2}$. Substituting for the norms on the right hand side of (100) yields the supremum estimate needed to prove Proposition 45. The Hölder estimate follows from similar, though more involved, calculations which can be found in [3]. The $\varepsilon^{-2 \beta}$ factor arises during the course of this latter calculation.

\subsection{The Size of the $F_{\alpha}(0,0,0)$ Term.}

The constants $C_{L}(\alpha)$ and $C_{N}(\alpha)$ required to estimate the size of the neighbourhood of surjectivity of the map $F_{\alpha}$ about $(0,0,0)$ according to the Inverse Function Theorem have now been found. It remains to estimate the size of $F_{\alpha}(0,0,0)$ in the $C_{\rho}^{0, \beta}$ Schauder norm. 
Proposition 46. Suppose $\alpha \leq A_{7}$. Then $F_{\alpha}(0,0,0)$ satisfies the bound

$$
\left|\rho^{2} F_{\alpha}(0,0,0)\right|_{C_{\rho}^{0, \beta}\left(\bar{M}_{\alpha}\right)} \leq C \alpha^{3-2 \beta-2 \beta / n},
$$

where the constant $C$ is independent of $\alpha$.

Proof. Abbreviate $F_{\alpha}(0,0,0)=\left\langle\bar{h}_{\alpha}^{*}(\operatorname{Im} \mathrm{d} z), \operatorname{Vol}_{\bar{M}_{\alpha}}\right\rangle_{\bar{M}_{\alpha}}$ by $E$. Thus by Proposition 14,

$$
|E|_{0, \bar{M}_{\alpha}} \leq C \alpha .
$$

But it is also true that $E$ vanishes everywhere except inside the transition region $T_{1} \cup T_{2}$. Property 1 of the weight function $\rho$ implies that $|\rho(x)| \leq$ $C \varepsilon^{-\beta}\|x\|$ in the transition region. Since $\|x\| \leq C \alpha$ there,

$$
\left|\rho^{2} E\right|_{0, \bar{M}_{\alpha}} \leq C \alpha^{3} \varepsilon^{-2 \beta}
$$

where $C$ is a constant independent of $\alpha$. Using the relationship $\varepsilon \leq C \alpha^{1+1 / n}$ yields supremum estimate required to prove the proposition. The Hölder estimate can be estimated in a similar, though more involved manner. The relevant calculations can be found in [3].

\subsection{Invoking the Inverse Function Theorem.}

All four components of the proof of the Main Theorem are now in place: the injectivity of the linearized operator and the injectivity bound; the surjectivity of the linearized operator; the estimate on the nonlinearities of $F_{\alpha}$; and the size of $F_{\alpha}$ at zero. All that remains is to assemble these results and to conclude the proof of the Main Theorem.

\section{EXISTENCE AND REgUlaRity of $M_{\alpha}$}

Choose $\alpha \leq A_{7}$. The calculations of Section 5 show that the linearized operator $\mathrm{DF}_{\alpha}(0,0,0)$ is a bijection. Furthermore, the functional dependence of $C_{L}(\alpha)$ and $C_{N}(\alpha)$ on $\alpha$ have been estimated as well, in Sections 5 and 6 , respectively. Substitute these estimates, along with the estimate the size of $F_{\alpha}(0,0,0)$ found in Section 6.1, into the inequality (4) of the Inverse Function Theorem. One concludes that a solution of the equation $F_{\alpha}(u, a, b)=0$ in the space $\mathcal{B}_{1, \alpha} \times \mathbf{R}^{2}$ can be found if

$$
C \alpha^{3-2 \beta-2 \beta / n} \leq \frac{\left(C_{L} \varepsilon^{2 \beta}\right)^{2}}{4 C_{N} \varepsilon^{-2-2 \beta}} .
$$


Rewrite (103) in terms of $\alpha$ alone: thus a solution exists whenever

$$
\alpha^{1-2 / n-8 \beta(1+1 / n)} \leq C .
$$

By examining the power of $\alpha$ in this inequality, it is easy to see that the inequality can be satisfied by all sufficiently small $\alpha$ so long as $n \geq 3$ and $\beta$ is itself chosen less than some $\alpha$-independent upper bound. Therefore, there always exists $\left(H_{\alpha}, \theta_{\alpha}, b_{\alpha}\right)$ solving the equation $F_{\alpha}(H, \theta, b)=0$ provided $\alpha$ is smaller than some upper bound. Note that the (103) can not be satisfied for all sufficiently small $\alpha$ in the case $n=2$.

The solution $\left(H_{\alpha}, \theta_{\alpha}, b_{\alpha}\right)$ that has been found belongs to $C_{\rho}^{2, \beta}\left(\bar{M}_{\alpha}\right) \times \mathbf{R}^{2}$ and thus $H_{\alpha}$ is smooth by standard elliptic regularity theory [22]. By the definition of the map $F_{\alpha}$, this means that the submanifold

$$
M_{\alpha} \equiv \phi_{b_{\alpha}}^{1} \circ \phi_{\left(H_{\alpha}\right)_{e}}^{1}\left(\bar{M}_{\alpha}\right)
$$

is a smooth, minimal Lagrangian submanifold calibrated by the form $\operatorname{Re}\left(\mathrm{e}^{\mathrm{i} \theta_{\alpha}} \mathrm{d} z\right)$ and has boundary lying on the scaffold $W_{\alpha}=\phi_{b}^{1}(W)$.

EMBEDDEDNESS OF $M_{\alpha}$

According to the Inverse Function Theorem, the solution of the equation $F_{\alpha}\left(H_{\alpha}, \theta_{\alpha}, b_{\alpha}\right)=0$ satisfies the estimate:

$$
\left\|H_{\alpha}, \theta_{\alpha}, b_{\alpha}\right\|_{C_{\rho}^{2, \beta}\left(\bar{M}_{\alpha}\right) \times \mathbf{R}^{2}} \leq \frac{C_{L} \varepsilon^{2 \beta}}{2 C_{N} \varepsilon^{-2-2 \beta}}=C \varepsilon^{2+4 \beta}
$$

for some geometric constant $C$ independent of $\alpha$. This, in turn, implies that

$$
\left|\rho \nabla H_{\alpha}\right|_{0, \bar{M}_{\alpha}} \leq C \varepsilon^{2+4 \beta} \quad \text { and } \quad\left|b_{\alpha}\right| \leq C \varepsilon^{2+4 \beta} .
$$

Both these quantities tend towards zero as $\alpha \rightarrow 0$.

In order to determine whether $M_{\alpha}=\phi_{b_{\alpha}}^{1} \circ \phi_{\left(H_{\alpha}\right)_{e}}^{1}\left(\bar{M}_{\alpha}\right)$ is an embedded submanifold of $\mathbf{R}^{2 n}$, it is enough to show that the Hamiltonian diffeomorphism $\phi_{b_{\alpha}}^{1} \circ \phi_{\left(H_{\alpha}\right)_{e}}^{1}$ deforms $\bar{M}_{\alpha}$ in a sufficiently small manner in the $C^{1}$ sense. But this is now a consequence of the fact that the Hamiltonian flow is a smooth function of the Hamiltonian and its first derivatives. The proof of the Main Theorem is now complete.

ACKnowledgements: I would like to thank my Ph.D. advisor at Stanford University, Rick Schoen, for his patience, insight and confidence in me while I was carrying out the research for this paper. I would also like to thank Rafe Mazzeo and Leon Simon for their generous assistance, as well as Justin Corvino and Vin de Silva for their inspirations, ideas, and careful proofreading. Finally, I would like to thank Yng-Ing Lee for discovering and helping me to overcome certain mathematical difficulties. 


\section{References.}

[1] R. Abraham, J. E. Marsden, and T. Ratiu, Manifolds, Tensor Analysis, and Applications, second ed., Springer-Verlag, New York, 1988.

[2] Adrian Butscher, Deformations of minimal Lagrangian submanifolds with boundary, Submitted to Proceedings of the AMS, August 2001. Preprint math.DG/0110052.

[3] Deformation Theory of Minimal Lagrangian Submanifolds, Ph.D. thesis, Stanford University, 2000.

[4] David Gilbarg and Neil S. Trudinger, Elliptic Partial Differential Equations of Second Order, second ed., Springer-Verlag, Berlin, 1983.

[5] Reese Harvey, Spinors and Calibrations, Academic Press, 1990.

[6] Reese Harvey and H. Blaine Lawson, Jr., Calibrated geometries, Acta Math. 148 (1982), 47-157.

[7] Mark Haskins, Constructing Special Lagrangian Cones, Ph.D. thesis, University of Texas at Austin, 2000.

[8] Nigel J. Hitchin, The moduli space of special Lagrangian submanifolds, Ann. Scuola Norm. Sup. Pisa Cl. Sci. (4) 25 (1997), no. 3-4, 503-515 (1998), Dedicated to Ennio De Giorgi.

[9] Dominic Joyce, Lectures on Calabi-Yau and special Lagrangian geometry, math.DG/0108088.

[10] Compact Riemannian 7-manifolds with holonomy $G_{2}$. I, II, J. Differential Geom. 43 (1996), no. 2, 291-328, 329-375.

[11] Nikolaos Kapouleas, Constant mean curvature surfaces in Euclidean spaces, Proceedings of the International Congress of Mathematicians, Vol. 1, 2 (Zürich, 1994) (Basel), Birkhäuser, 1995, pp. 481-490.

[12] On desingularizing the intersections of minimal surfaces, Proceedings of the 4th International Congress of Geometry (Thessaloniki, 1996), Giachoudis-Giapoulis, Thessaloniki, 1997, pp. 34-41.

[13] Gary Lawlor, The angle criterion, Invent. Math. 95 (1989), 437-446.

[14] Yng-Ing Lee, Embedded special Lagrangians in calabi-yau manifolds, Preprint, 2001. 
[15] Rafe Mazzeo, Frank Pacard, and Dan Pollack, CMC Surfaces, To appear.

[16] Robert C. McLean, Deformations of calibrated submanifolds, Comm. Anal. Geom. 6 (1998), no. 4, 705-747.

[17] David R. Morrison, Mathematical aspects of mirror symmetry, Complex Algebraic Geometry (Park City, UT, 1993), Amer. Math. Soc., Providence, RI, 1997, pp. 265-327.

[18] R. Schoen and J. Wolfson, Minimizing volume among Lagrangian submanifolds, Differential Equations: La Pietra 1996 (Shatah Giaquinta and Varadhan, eds.), Proc. of Symp. in Pure Math., vol. 65, 1999, pp. 181-199.

[19] Richard Schoen, Lecture Notes in Geometric PDEs on Manifolds, Course given in the Spring of 1998 at Stanford University.

[20] Leon Simon, Lecture Notes in PDE Theory, Course given in 1997 at Stanford University.

[21] Lectures on Geometric Measure Theory, Australian National University Centre for Mathematical Analysis, Canberra, 1983.

[22] Michael E. Taylor, Partial Differential Equations. III, Springer-Verlag, New York, 1997, Nonlinear equations.

Max Planck Institute for Gravitational Physics

butscher@aei-potsdam.mpg.de

Received December 5, 2001. 
